University of Tennessee Health Science Center

UTHSC Digital Commons

$12-2009$

\title{
Identification of the Downstream Effector Genes Involved in HOXB4-Induced Expansion of Hematopoietic Progenitor Cells
}

Jie Jiang

University of Tennessee Health Science Center

Follow this and additional works at: https://dc.uthsc.edu/dissertations

Part of the Medical Cell Biology Commons

\section{Recommended Citation}

Jiang, Jie , "Identification of the Downstream Effector Genes Involved in HOXB4-Induced Expansion of Hematopoietic Progenitor Cells" (2009). Theses and Dissertations (ETD). Paper 137. http://dx.doi.org/ 10.21007/etd.cghs.2009.0154.

This Dissertation is brought to you for free and open access by the College of Graduate Health Sciences at UTHSC Digital Commons. It has been accepted for inclusion in Theses and Dissertations (ETD) by an authorized administrator of UTHSC Digital Commons. For more information, please contact jwelch30@uthsc.edu. 


\title{
Identification of the Downstream Effector Genes Involved in HOXB4-Induced Expansion of Hematopoietic Progenitor Cells
}

\author{
Abstract \\ Overexpression of HOXB4, a member of Homeobox transcription factor family, promotes expansion of \\ hematopoietic stem and progenitor cells bothin vivo and in vitro. However, the molecular mechanisms \\ underlying this effect are not well understood. In order to identify direct target genes of HOXB4 in primary \\ murine hematopoietice progenitor cells, we induced HOXB4 function in lineage-negative, murine bone \\ marrow cells, using a tamoxifen-inducibleHOXB4-ER ${ }^{T 2}$ fusion protein. Seventy seven genes with \\ differentially changed expression in early response to HOXB4 have been identified as candidate target \\ genes. Among them, we show that Hemogen (Hemgn), encoding a nuclear protein specifically expressed \\ in hematopoietic stem and progenitor cells, is a direct transcriptional target of HOXB4, and that HOXB4 \\ binds to the promoter region of Hemgn. More importantly, when overexpressed in bone marrowcells, \\ Hemgn promotes expansion of 5-fluorouracil (5-FU) treated bone marrow cells in both liquid and semi- \\ solid cultures, recapitulating the effects of HOXB4. Furthermore, both Hemgn and HOXB4 can protect \\ bone marrow cells from apoptosis. Our results identify an important direct transcriptional target of \\ HOXB4 that can confer expansion of primitive myeloid progenitor cells. \\ Document Type \\ Dissertation \\ Degree Name \\ Doctor of Philosophy (PhD) \\ Program \\ Biomedical Sciences \\ Research Advisor \\ Brian P. Sorrentino, M.D. \\ Keywords \\ hematopoietic progenitor cells, Hemgn, HOXB4, self-renewal \\ Subject Categories \\ Medical Cell Biology | Medical Sciences | Medicine and Health Sciences \\ Comments \\ One year embargo expired December 2010
}

This dissertation is available at UTHSC Digital Commons: https://dc.uthsc.edu/dissertations/137 


\title{
IDENTIFICATION OF THE DOWNSTREAM EFFECTOR GENES INVOLVED IN HOXB4-INDUCED EXPANSION OF HEMATOPOIETIC PROGENITOR CELLS
}

\author{
A Dissertation \\ Presented for \\ The Graduate Studies Council \\ The University of Tennessee \\ Health Science Center
}

\author{
In Partial Fulfillment \\ Of the Requirement for the Degree \\ Doctor of Philosophy \\ From The University of Tennessee
}

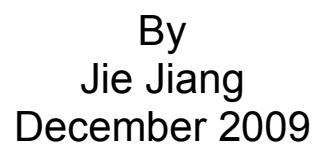


Copyright $@ 2009$ by Jie Jiang All rights reserved 


\section{DEDICATION}

This dissertation is dedicated to My parents, Mali Wu and Zhiyuan Jiang

My husband, Ji Zhang

And My daughter Ariel Q. Zhang for their love and support 


\section{ACKNOWLEDGEMENTS}

First, I would like to thank my mentor, Dr. Brian P. Sorrentino, for his guidance, support and help throughout my graduate study. His appealing ideas and patient training made my project possible.

I would also like to express my deep gratitude to my other committee members, Dr. Paul A. Ney, Dr. Guillermo Oliver, Dr. Lawrence Pfeffer and Dr. Edward Park for their suggestions and discussion, which have benefited me a great deal.

Last, I would like to thank my previous colleague, Dr. Yan Shou, and my colleagues, Dr. Sheng Zhou, Hui Yu, Dr. Taihe Lu, Peng Gao, Dr. Soghra Fatima, Dr. Zhijun Ma, Dr. Louise Treanor, Dr. Disha Mody, Dr. Michael Greene, for their kind help.

This work can not be achieved without the technical support from Dr. Geoffrey Neale in Hartwell center for Bioinformatics and Biotechnology, the Flow cytometry laboratory, the Animal Resource Center, and the Experimental Hematology Division in St. Jude Children's Research Hospital. 


\begin{abstract}
Overexpression of HOXB4, a member of Homeobox transcription factor family, promotes expansion of hematopoietic stem and progenitor cells both in vivo and in vitro. However, the molecular mechanisms underlying this effect are not well understood. In order to identify direct target genes of HOXB4 in primary murine hematopoietice progenitor cells, we induced HOXB4 function in lineage-negative, murine bone marrow cells, using a tamoxifen-inducible HOXB4-ER ${ }^{\mathrm{T} 2}$ fusion protein. Seventy seven genes with differentially changed expression in early response to HOXB4 have been identified as candidate target genes. Among them, we show that Hemogen (Hemgn), encoding a nuclear protein specifically expressed in hematopoietic stem and progenitor cells, is a direct transcriptional target of HOXB4, and that HOXB4 binds to the promoter region of Hemgn. More importantly, when overexpressed in bone marrow cells, Hemgn promotes expansion of 5-fluorouracil (5-FU) treated bone marrow cells in both liquid and semi-solid cultures, recapitulating the effects of HOXB4. Furthermore, both Hemgn and HOXB4 can protect bone marrow cells from apoptosis. Our results identify an important direct transcriptional target of HOXB4 that can confer expansion of primitive myeloid progenitor cells.
\end{abstract}




\section{TABLE OF CONTENTS}

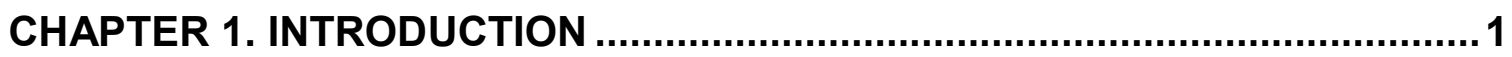

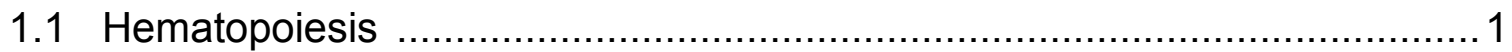

1.2 Hematopoietic Stem and Progenitor Cells ........................................ 1

1.3 Factors in Hematopoietic Stem and Progenitor Cell Self-Renewal ............... 3

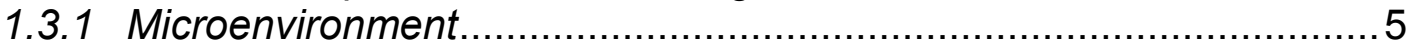

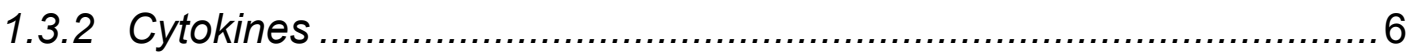

1.3.3 Cell Cycle and Apoptotic Regulators........................................... 6

1.3.4 Signal Transducer and Transcriptional Factors...............................

1.4 Expanding Hematopoietic Stem Cells for Transplantation ......................... 8

1.5 Characteristics of Hox Gene Family Members ..................................... 9

1.5.1 Functions of Hox Family Members in Hematopoiesis .....................11

1.5.2 Biochemical Characteristics of HOXB4 and Other Hox Family Members ............................................................................ 12

1.5.3 Hox Downstream Target Genes.............................................. 14

1.6 Role of HOXB4 in Hematopoietic Stem and Progenitor Cell Expansion ..... 16

1.6.1 Overexpression of HOXB4 In Vivo .............................................. 16

1.6.2 Overexpression of HOXB4 In Vitro............................................... 18

1.6.3 ES Cell Specification .................................................................20

1.6.4 Potential Toxicity of HOXB4 Overexpression ...............................20

1.6.5 Hoxb4 Knockout and Other Hox-Deficient Mice ............................21

1.6.6 Mechanisms of HOXB4 Function .................................................22

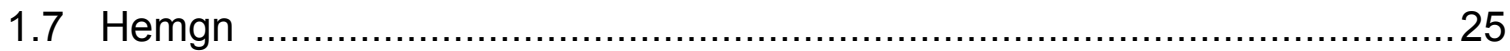

1.7.1 Expression Pattern and Biochemical Characteristics of Hemgn ......25

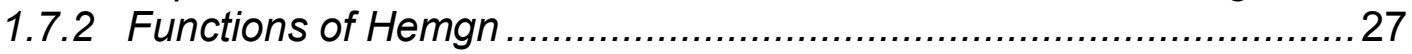

\section{CHAPTER 2. IDENTIFICATION OF EARLY AND POTENTIAL DIRECT} TARGET GENES OF HOXB4 USING AN INDUCIBLE HOXB4-ER ${ }^{\text {T2 }}$

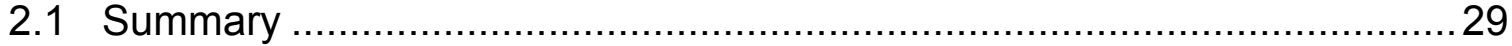

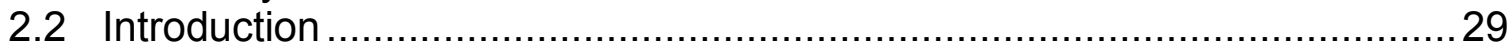

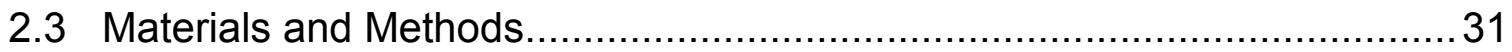

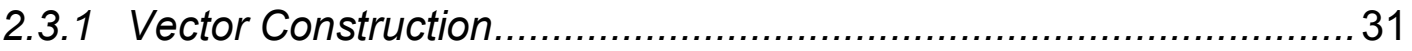

2.3.2 Retroviral Vector Preparation .................................................... 31

2.3.3 Separation, Transduction of Lin Bone Marrow Cells and Analysis .. 31

2.3.4 TAM Induction of HOXB4-ER ${ }^{T 2}$ and Myeloid Progenitor Assays...... 32

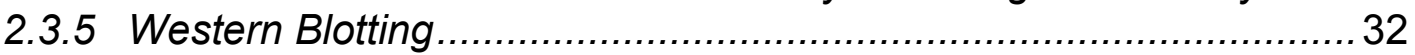

2.3.6 Immunofluorescence Staining .................................................. 32

2.3.7 RNA Isolation, Affymetrix Array Hybridization and Analysis............. 33

2.3.8 Gene Expression Validation ........................................................ 33

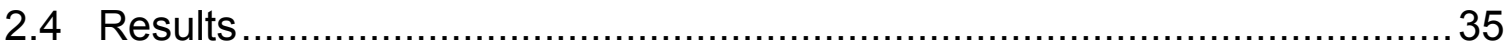


2.4.1 Retroviral Constructs and Expression of HOXB4-ER ${ }^{T 2}$ Fusion Protein.

2.4.2 TAM Inducible Nuclear-Translocation of HOXB4-ER $R^{T 2 \ldots \ldots \ldots \ldots \ldots \ldots \ldots \ldots \ldots \ldots . .35}$

2.4.3 TAM Inducible HOXB4-ER ${ }^{T 2}$ Activity Promoted Hematopoietic Progenitor Cell Expansion In Vitro

2.4.4 Identification of HOXB4 Early Downstream Targets by Expression Microarrays ............................................................................ 41

2.4.5 Validation of the Selected HOXB4 Target Genes........................... 41

2.4.6 Hemgn mRNA Expression Level in Bone Marrow and c-kit ${ }^{+}$Scal- $1^{+}$Lin $^{-}$Cells from Hoxb4 ${ }^{-/-}$Mice........................................ 52

2.5 Discussion

\section{CHAPTER 3. DEMONSTRATION THAT HEMGN IS A DIRECT} TRANSCRIPTIONAL TARGET OF HOXB4 .............................................56

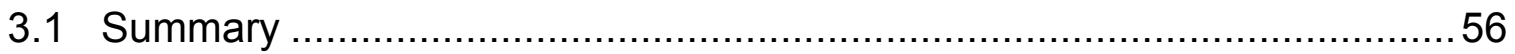

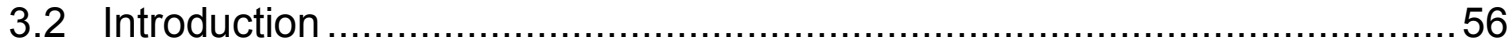

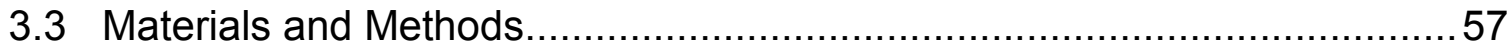

3.3.1 Transient Cotransfection Luciferase Assay .................................57

3.3.2 Electrophoretic Mobility Shift Assay (EMSA)...............................58

3.3.3 Separation and Transduction of 5-FU Treated Bone Marrow Cells.. 58

3.3.4 Chromatin Immunoprecipitation (ChIP) Assay ............................58

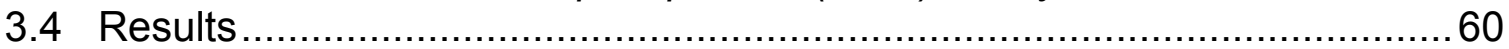

3.4.1 The Promoter of Hemgn Contains Potential HOXB4 Binding Motifs

3.4.2 The Promoter of Hemgn Was Transcriptionally Activated by HOXB4.....

3.4.3 HOXB4 Directly Bound to the Promoter Fragment of Hemgn

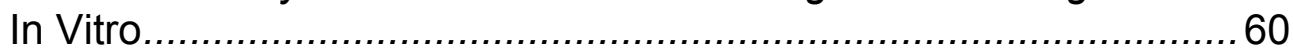

3.4.4 HOXB4 Was Recruited to the Promoter Region of Hemgn In Vivo .. 62

\section{CHAPTER 4. EXPRESSION OF HEMGN EXPANDS BONE MARROW AND HEMATOPOIETIC PROGENITOR CELLS AND PROTECTS AGAINST APOPTOSIS}

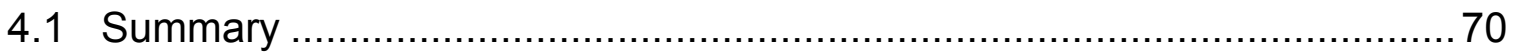

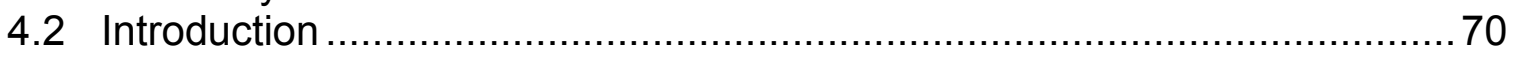

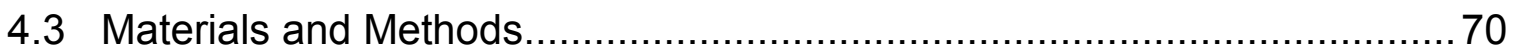

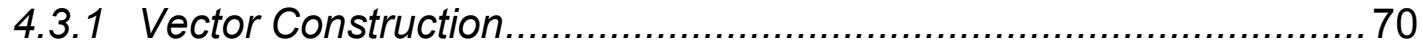

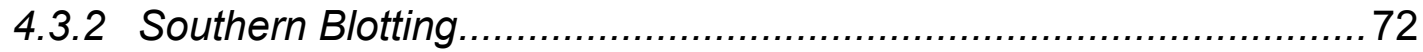

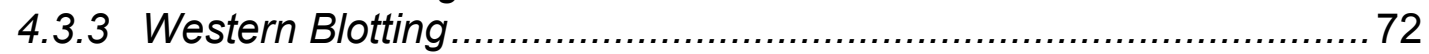

4.3.4 Transduction of 5-FU Treated Bone Marrow Cells .......................... 72

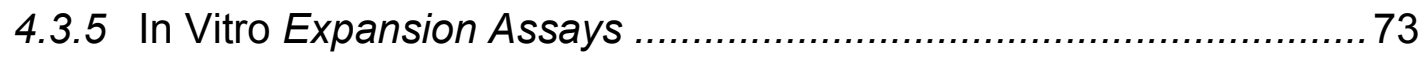

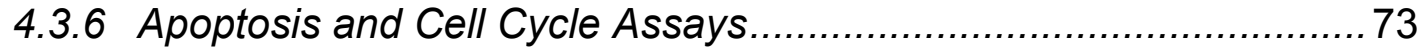

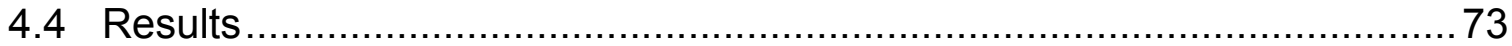


4.4.1 Expression of Hemgn in Transduced Cells .................................. 73

4.4.2 Hemgn Promoted Hematopoietic Cell Expansion in Liquid Culture.. 75

4.4.3 Hemgn Promoted Myeloid Progenitor Cell Expansion In Vitro .......75

4.4.4 Hemgn Protected Bone Marrow Cells from Apoptosis ................... 79

4.4.5 Hemgn Overexpression Has No Direct Effects on Cell Cycle in Hematopoietic Cells................................................................... 83

4.5 Discussion

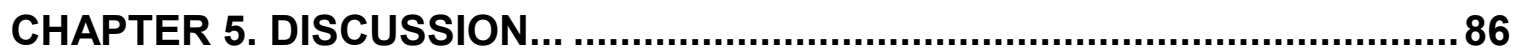

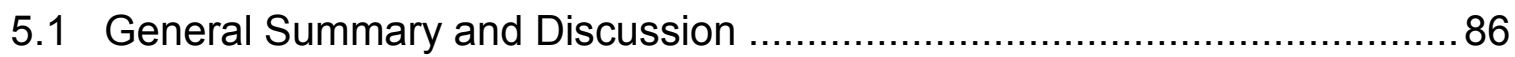

5.1.1 Target Genes Downstream of HOXB4 ...................................... 86

5.1.2 The Mechanism of Hemgn as a Functional Direct Target of HOXB4

5.1.3 Relationship between Hemgn Expression and Endogenous Hoxb4......

5.1.4 Upstream Regulation of Hemgn: Other Mechanisms ...................... 89

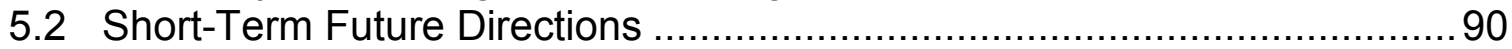

5.3 Long-Term Future Directions ............................................................ 91

5.3.1 Biochemical and Functional Characterization of Hemgn ................. 91

5.3.2 Potential Clinical Use of Hemgn................................................92

5.3.3 Identification of Other Direct Target Genes of HOXB4................... 92

5.4 Detailed Consideration of These Results and Their Impact on Understanding the Molecular Mechanisms of Hematopoietic Stem Cell

Expansion

5.4.1 Hematopoietic Stem Cell Self-Renewal and the Role of HOXB4 .....93

5.4.1.1 Survival Signals........................................................ 93

5.4.1.2 Proliferation Signals ................................................... 94

5.4.1.3 Anti-Differentiation Signals ........................................... 95

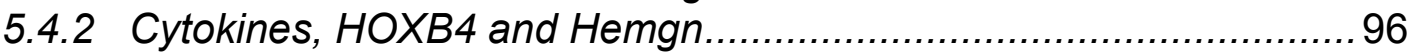

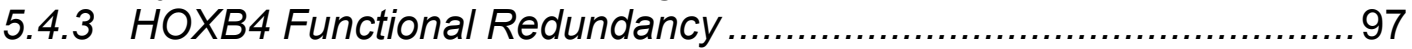

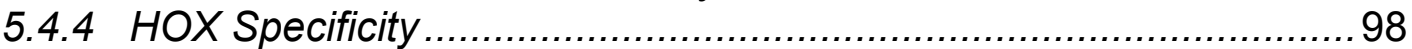

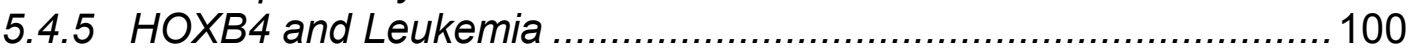

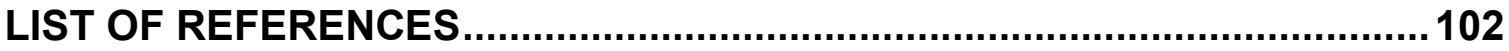

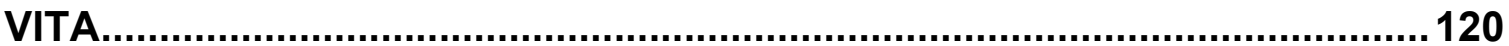




\section{LIST OF TABLES}

Table 2-1. The ordered gene-specific PCR primers used in real-time PCR validation analysis.

Table 2-2. Pearson correlation coefficients between the 8 experimental samples with probesets $>1$ present call in microarray.

Table 2-3. The 44 probesets upregulated by HOXB4-ER ${ }^{\top 2}$. 44

Table 2-4. The 33 probesets downregulated by HOXB4-ER ${ }^{T 2}$ 46 


\section{LIST OF FIGURES}

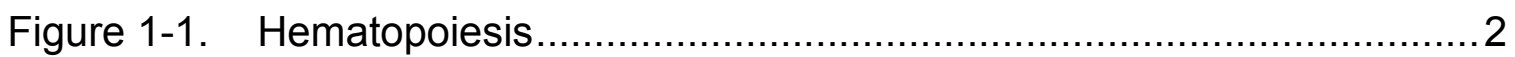

Figure 1-2. Hematopoietic stem cell outcomes ........................................ 4

Figure 1-3. Genomic organization and collinear expression patterns of Drosophila Hom genes and mammalian Hox genes ....................10

Figure 1-4. Biochemical characteristics of Hox proteins .............................13

Figure 1-5. HOXB4 has preference for the nucleotides flanking the "TAAT" core binding sequence..................................................... 15

Figure 1-6. HOXB4 induces expansion of hematopoietic stem cells in vivo. ...17

Figure 1-7. HOXB4 induces expansion of hematopoietic stem cells in vitro .... 19

Figure 1-8. Redundancy of HOX4 paralogs in promoting hematopoietic stem and progenitor cell expansion................................................24

Figure 1-9. Expression and localization of Hemgn...................................26

Figure 2-1. Retroviral constructs and expression of HOXB4 and HOXB4-

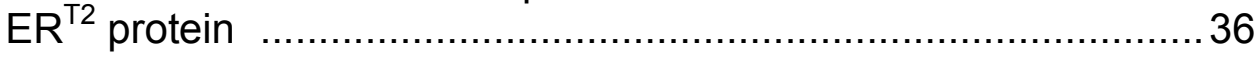

Figure 2-2. TAM-induced cytosol to nucleus translocation of HOXB4-ER ${ }^{\top 2}$ in Lin bone marrow cells confirmed by confocal microscopy...

Figure 2-3. Schematic illustration of myeloid progenitor secondary CFU-C expansion assays............................................... 38

Figure 2-4. Effect of TAM-induced HOXB4-ER ${ }^{\top 2}$ activity in vitro ......................39

Figure 2-5. Secondary myeloid progenitor colonies from HOXB4-ER ${ }^{\top 2}$ overexpressing Lin $^{-}$bone marrow cells were GFP positive under fluorescence microscopy.

Figure 2-6. Schematic illustration of the inducible HOXB4-ER ${ }^{\mathrm{T} 2}$ system for target screening in Lin ${ }^{-}$bone marrow cells 
Figure 2-7. Hierarchical cluster diagram and heat map of the 77 candidate probesets selected from 4 biologically-independent experiments using the $\log _{2}$-ratio of TAM-induced HOXB4-ER ${ }^{\mathrm{T} 2}$ vs. TAMinduced GFP expression values observed within each of the 4 experiments

Figure 2-8. Hierarchical cluster diagram and heat map of the 77 candidate probesets selected from 4 biologically-independent experiments using the normalized z-score

Figure 2-9. Functional classification of the 77 probesets by using gene ontology annotations 50

Figure 2-10. Quantitative real-time PCR confirmation of the expression change of the selected 8 candidate target genes from independent experiments with TAM treatment for 6 hours or 12 hours in HOXB4-ER ${ }^{\text {T2 }}$ vector-transduced and GFP control vector-transduced Lin $^{\top}$ bone marrow cells.

Figure 2-11. Quantitative real-time PCR detection of relative expression level of Hemgn mRNA in whole bone marrow or c-kit ${ }^{+} \mathrm{Scal}-1^{+} \mathrm{Lin}^{-}$ (KSL) cells from $\mathrm{Hoxb4}^{-/-}$mice compared to wild type (WT) mice..53

Figure 3-1. Schematic illustration of the multiple putative HOXB4 binding sites on the promoter region of Hemgn (site 1, 2, 3 and 4)

Figure 3-2. HOXB4-dependent increase in promoter activity of Hemgn in MEL cells

Figure 3-3. HOXB4-specific binding caused band shift of probes containing the putative HOXB4 binding site (site 1) in EMSA

Figure 3-4. Immunoprecipitation and western blotting detection of HOXB4 protein from GPE+86 cells overexpressed with GFP control or HOXB4 with monoclonal anti-HOXB4 antibody (I12)

Figure 3-5. Sonicated chromatin fragments from GFP or HOXB4transduced bone marrow cells for ChIP assay

Figure 3-6. Semi-quantitative PCR detected enrichment of the promoter fragments of Hemgn by HOXB4 specific binding following ChIP assay in transduced bone marrow cells .

Figure 3-7. Quantitative real-time PCR detected relative enrichment of individual regions in Hemgn promoter by HOXB4 following ChIP assay in transduced bone marrow cells 
Figure 4-1. Hemgn-expressing retroviral vector

Figure 4-2. Integration, and protein expression of Hemgn and its GFP reporter in GPE+86 vector producer and bone marrow cells

Figure 4-3. Experimental design of in vitro expansion assays .......................76

Figure 4-4. Growth advantage of Hemgn-overexpressing bone marrow cells in liquid culture

Figure 4-5. Growth advantage of Hemgn-overexpressing hematopoietic progenitor cells in methylcellulose culture.

Figure 4-6. Flow cytometry-detected GFP percentage of bone marrow cells collected from primary and secondary CFU-C colonies

Figure 4-7. Flow cytometry analysis for apoptosis in GFP positive vs. negative population of transduced bone marrow cells in cytokine-depleted and serum-reduced medium

Figure 4-8. Quantification and statistics of three independent experiments for apoptosis in GFP positive vs. negative transduced bone marrow cells in cytokine-depleted and serumreduced medium

Figure 4-9. DNA content of vector-transduced bone marrow cells cultured for 6 days post transduction 


\section{CHAPTER 1. INTRODUCTION}

\subsection{Hematopoiesis}

Hematopoiesis is a process of successive formation and development of blood cells derived from hematopoietic stem cells. ${ }^{1}$ Hematopoietic stem cells can generate multi-lineage or single-lineage commited hematopoietic progenitor cells for mature blood cells production. Within multi-lineage hematopoietic progenitor cells, common lymphoid progenitors (CLPs) can give rise to B and T lymphocytes; on the other hand, common myeloid progenitors (CMPs) can give rise to various multi- or single-lineage hematopoietic progenitor cells to produce platelets, erythrocytes, granucytes and monocytes (Figure 1-1). ${ }^{2-6}$ In humans, hematopoiesis originates in blood islands in the embryonic yolk sac; then switches to fetal liver, and then to spleen as adult hematopoietic stem cells migrate to these locations. In adults, bone marrow is the major site of hematopoiesis.

Hematopoietic cells are short-lived, thus, to maintain cellular homeostasis, continuous generation of hematopoietic cells is required. This process is regulated by a complex balance between cell self-renewal, differentiation and apoptosis. $^{7-10}$

\subsection{Hematopoietic Stem and Progenitor Cells}

Hematopoietic stem cells were originally identified by Till and McCulloch in mouse transplantation assays. ${ }^{11}$ Hematopoietic stem cells are self-renewing cells that have the ability to differentiate into all of the blood cell lineages. ${ }^{12 ; 13}$

There are two kinds of hematopoietic stem cells that can be isolated in mice embryonic hematopoietic stem cells and adult hematopoietic stem cells. ${ }^{14 ; 15}$ Embryonic hematopoietic stem cells develop in the yolk sac at day 9.0 of embryogenesis (E9.0) and can reconstitute hematopoiesis after injection into neonatal mice. Adult hematopoietic stem cells emerge in the aorta, gonads, and mesonephros (AGM) region around E10.0 and can reconstitute hematopoiesis in lethally-irradiated adult mice. The most stringent method to evaluate and quantify hematopoietic stem cells is to transplant them into lethally-irradiated recipient animals. In vivo studies show that one single hematopoietic stem cell is sufficient to restore the whole blood system in lethally irradiated recipient mice. ${ }^{16}$ Hematopoietic stem cells can be isolated from bone marrow by various methods. One approach is to use antibodies that enrich for cells expressing hematopoietic stem cell-associated cell surface markers (e.g., c-kit, Sca-1, Thy1.1) and are depleted of cells expressing mature lineage markers (e.g., Ter119, B220, CD3, Mac-1, Gr-1). Hematopoietic stem cell activity resides in a very small proportion of bone marrow cells, characterized by the Lin $^{-} \mathrm{CD} 41^{-} \mathrm{CD} 48^{-} \mathrm{CD} 150^{+}$or 


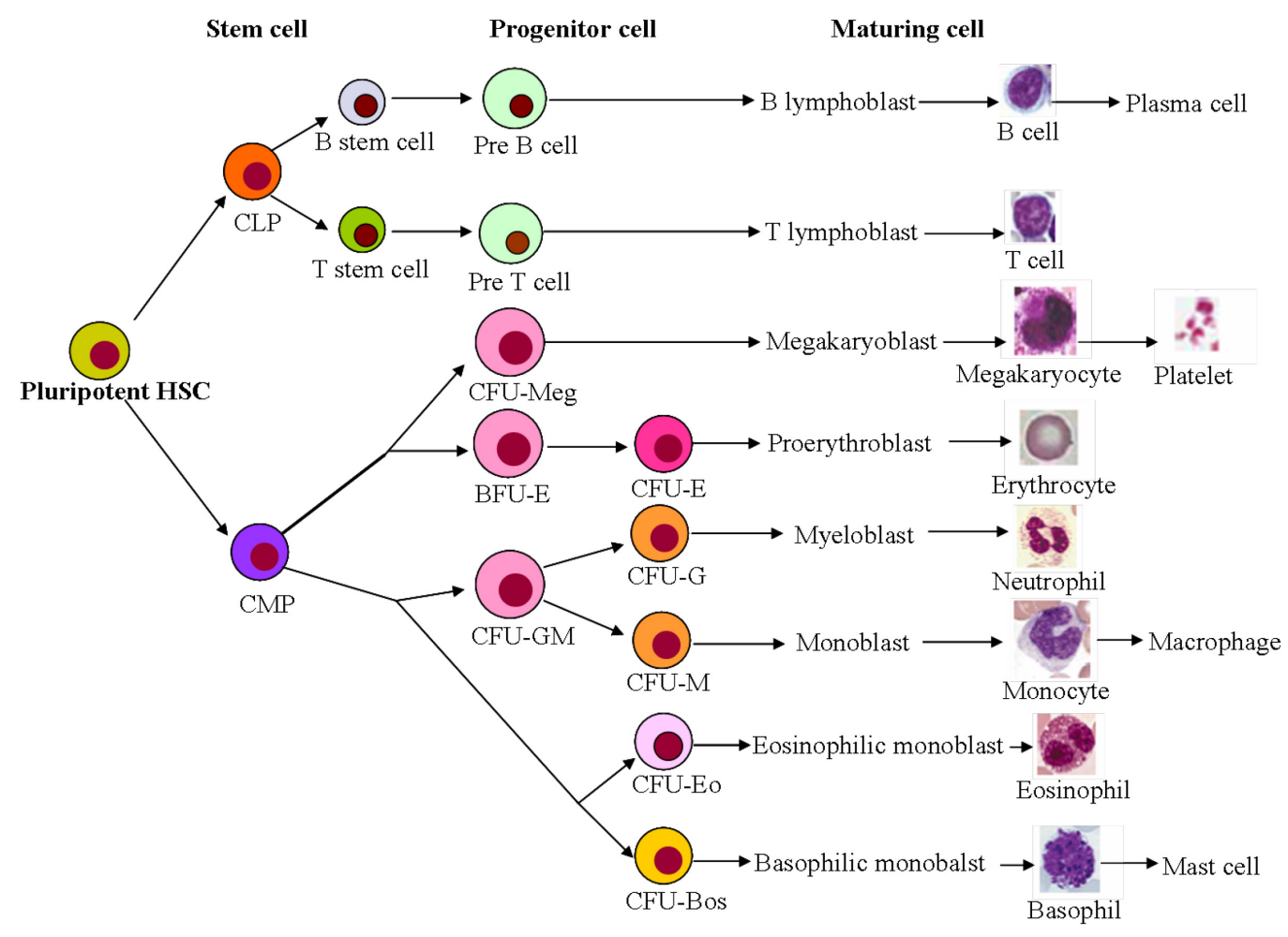

Figure 1-1. Hematopoiesis. 
Lin-Sca $1^{+} \mathrm{CD} 117^{+}$(LSK) phenotype. ${ }^{17}$ After injection into lethally-irradiated recipient mice, these cells migrate to appropriate microenvironmental niches and undergo rapid expansion and differentiation. In the short term, they provide radioprotection by generating mature erythroid and myeloid cells that are necessary for survival. Hematopoietic stem cells also self-renew to generate more stem cells and sustain steady-state hematopoiesis during the lifetime of an organism. ${ }^{18 ; 19}$ Hematopoietic stem cells are less well-defined in humans due to the lack of an equivalent repopulating assay suitable for quantitate experiments, although xenograft studies in immunodeficient mice are increasingly used for this purpose.

Under homeostatic conditions in vivo, most hematopoietic stem cells and some hematopoietic progenitor cells are in the $\mathrm{G}_{0}$ phase of cell cycle. Many of the late and more committed hematopoietic progenitor cells are actively proliferating to generate mature offsprings. Once hematopoietic stem and progenitor cells are stimulated to enter cell cycle, several outcomes are possible (Figure 1-2).${ }^{20}$ First, they can divide into committed daughter cells in the process of differentiation. Under stressful conditions in vivo, such as bleeding or infection, this process can generate mature blood cells to replenish the loss or to increase the immune response. Under unfavorable in vitro culture conditions, hematopoietic stem and progenitor cells can also undergo differentiation and lose their original stem and progenitor cell characteristics. Hematopoietic stem cells can also undergo selfrenewal by dividing into two daughter cells which retain the biological properties of the original cell. Third, under homeostatic conditions in vivo, apoptosis can occur and serve to balance the proliferation and differentiation processes. ${ }^{21 ; 22}$

Committed hematopoietic progenitor cells can enter a transient state of rapid cell expansion. After exhausting their expansion potential, these cells withdraw from cell cycle and progressively acquires characteristics of a predetermined blood cell type. ${ }^{20}$ The commitment of hematopoietic progenitor cells depends on specific growth factors and particular microenvironmental signals. Hematopoietic stem cells reside and develop on stroma cells, such as fat cells, endothelial cells, fibroblasts, and macrophages. These nonhematopoietic cells provide microenvironmental signals that support the growth and differentiation of hematopoietic cells. ${ }^{23-30}$

\subsection{Factors in Hematopoietic Stem and Progenitor Cell Self-Renewal}

While the phenotypic and functional properties of hematopoietic stem cells have been extensively characterized, a fundamental question that remains is how self-renewal is regulated. One of the most essential issues in stem cell research is to understand the mechanism of hematopoietic stem and progenitor cells self-renewal, due to its significance in both basic research and clinical applications. Identifying the molecules for promoting hematopoietic stem cells self-renewal may eventually allow the generation of expanded populations of 


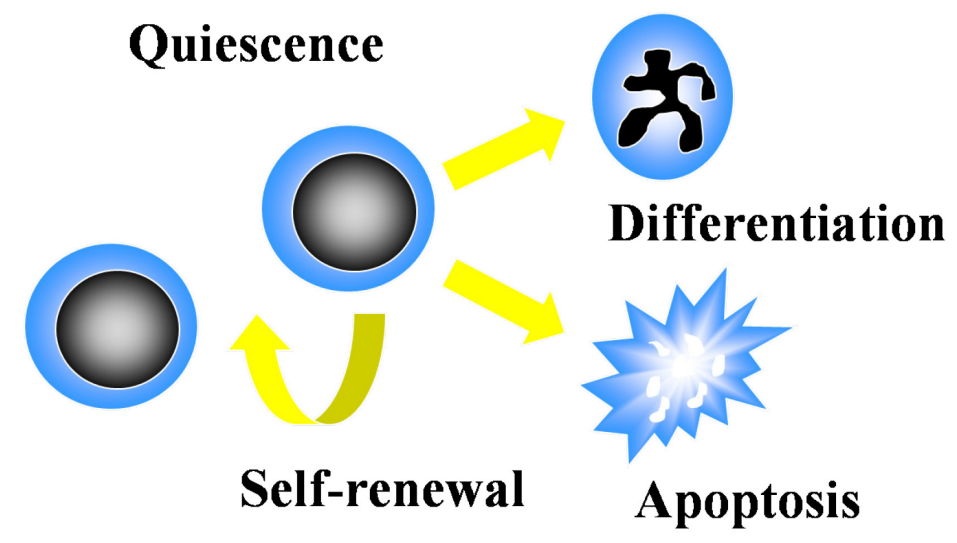

Figure 1-2. Hematopoietic stem cell outcomes. 
hematopoietic stem cells in vitro that can be used therapeutically. Self-renewal requires the combination of both survival and proliferation pathways, and inhibition of apoptosis and differentiation signals, which appears to be controlled both by extrinsic signals from the microenvironment and by intrinsic genetic programs ${ }^{31 ; 32}$ For cell-extrinsic pathways, recent results suggest that the osteoblastic niche could impact hematopoietic stem cell function. It has been proposed that hematopoietic stem cells reside in a specific microenvironment, osteoblastic niche, which is composed of the non-hematopoietic stroma cells, locally released cytokines, and the extracellular matrix. All these factors act together to sustain hematopoietic stem cells in bone marrow. ${ }^{24 ; 26-30 ; 33 ; 34}$ For cellintrinsic genetic programs involved in hematopoietic stem and progenitor cell self renewal, cell cycle regulators, anti-apoptotic proteins, signal transducers, and transcription factors have been proven to play essential roles.

\subsubsection{Microenvironment}

Genetic studies have demonstrated that osteoblasts are a crucial cellular element of the hematopoietic stem cell niche in bone marrow. ${ }^{35 ; 36}$ Hematopoietic stem cells receive critical signals from stroma cells and extracellular matrix (ECM) for expansion and differentiation. Some components of the ECM can bind to growth factors produced by stroma cells and also can bind to glycoproteins expressed on the cell surface of hematopoietic stem and progenitor cells. The interaction between hematopoietic stem cells and a component of ECM-fibronectin (FN) can stimulate growth and survival. ${ }^{37}$ This interaction is augmented by angiopoietin 1(Ang-1) which is expressed by both osteoblasts and hematopoietic stem cells. This cytokine acts through the hematopoietic stem cellsurface receptor Tie-2 for maintaining the self-renewal ability. ${ }^{38 ; 39}$ The direct interaction between hematopoietic stem cells and stroma cells or ECM in the niche can also promote hematopoietic stem cell self-renewal. For example, activation of Notch transmembrane receptors expressed in hematopoietic stem cells by their corresponding ligands expressed on stroma cells such as Jagged 1 and Jagged 2, can promote the self-renewal process. ${ }^{40-44}$ Inhibition of Notch signaling in adult hematopoietic stem cells led to accelerated differentiation in vitro and depletion of stem cells in vivo. ${ }^{45}$ Retroviral overexpression of a dominant active form of Notch established immortalized hematopoietic stem cells that were dependent on a cytokine cocktail of SCF, IL-6, IL-11 and Flt3 ligand. Enhanced activation of Notch1 or Notch4 (also known as Int3) contributed to increased self-renewal ability and consequent differentiation inhibition of hematopoietic stem cells, providing further evidence of the role of Notch signaling. ${ }^{46 ; 47}$ The Wnt pathway, which functions during development for initiating cellular events such as cell proliferation and differentiation, is also shown to be involved in this process. For example, it has been shown that Wnt3A and $\beta$ catenin play key roles in hematopoietic stem cell self-renewal. ${ }^{4849}$ 


\subsubsection{Cytokines}

Cytokines are a broad family of proteins that mediate positive and negative signals that control cellular quiescence, apoptosis, proliferation, and differentiation. In general, cytokines function by engaging a specific receptor and activating a variety of signaling pathways. ${ }^{50}$ Among the different cytokines, stem cell factor (SCF), interleukin-3(IL-3), IL-6, Flt3 ligand, thrombopoietin (TPO) have been shown to modestly expand hematopoietic stem cells in vitro. ${ }^{51-54}$ The combination of SCF, IL-6, TPO and FIt3 ligand can promote transient hematopoietic stem cell expansion in vitro. ${ }^{55}$ Particularly, the combination of SCF, IL-3 and IL- 6 was demonstrated to increase the retrovirus-mediated gene transduction efficiency in murine hematopoietic stem cells. ${ }^{56 ; 57}$ Some cytokines such as IL-3 and SCF induce cell proliferation of hematopoietic stem and progenitor cells, while others such as Flt-3 ligand and kit ligand protect cells from apoptosis and sensitize them to the effects of growth promoting cytokines ${ }^{58-60}$ In contrast, TGF- $\beta 1$ can inhibit the growth of hematopoietic stem cells. ${ }^{61 ; 62}$ The inhibition of TGF- $\beta 1$ production by antisense oligos can promote the release of hematopoietic stem cells from quiescence ${ }^{63 ; 64}$ Another TGF- $\beta$ family member, bone morphogenetic protein-4 (BMP4) and its upsteam regulator sonic hedgehog (shh) is able to induce hematopoietic stem cell self-renewal. ${ }^{65 ; 66}$

\subsubsection{Cell Cycle and Apoptotic Regulators}

A key mediator of hematopoietic stem cell maintenance is a G1 checkpoint regulator, the cyclin-dependent kinase inhibitor $\mathrm{p} 21^{\mathrm{cip} 1 / \text { waf1 }}(\mathrm{p} 21){ }^{67 ; 68} \operatorname{In} p 21^{-/-}$ mice, the number of hematopoietic stem cells was increased at steady state conditions but hematopoietic failure occurred under conditions of stress.

Hematopoietic stem cell self-renewal failure had been observed during the serial transplantation of $p 21^{-1-}$ cells. Another example is a cell cycle inhibitor, $\mathrm{p} 18^{\text {INK } 4 \mathrm{C}}$. The absence of $\mathrm{p} 18^{\mathrm{INK} 4 \mathrm{C}}$ led to increased hematopoietic stem cell self-renewal. ${ }^{6}$ Therefore, intricate control of the cell cycle machinery is required for self-renewal regulation.

A large body of evidence suggests that suppression of apoptosis is required for hematopoietic stem cell survival. Studies using transgenic mice constitutively expressing BCL2 (B-cell lymphoma 2 ) in all hematopoietic tissues provided evidence directly supporting this hypothesis. The forced expression of the $B c / 2$ oncogene resulted in increased numbers of transgenic hematopoietic stem cells in vivo and those cells showed a competitive advantage over wild type cells in competitive reconstitution experiments in vivo, ${ }^{10 ; 21 ; 22}$ suggesting that cell death plays a role in regulating the homeostasis of hematopoietic stem cells. Recently, Mcl1 (Myeloid cell leukemia 1), another anti-apoptotic Bcl2 family member, has also been shown to be required for hematopoietic stem and progenitor cell survival. ${ }^{70}$ These results indicate that anti-apoptotic signals may contribute to regulating hematopoietic stem cell numbers. 


\subsubsection{Signal Transducer and Transcriptional Factors}

The Pten (phosphatase and tensin homologue deleted on chromosome ten) tumor suppressor, a modulator of several major signaling pathways, has recently been implicated as a regulator of hematopoietic stem cell self-renewal and an initiator of leukemogenesis. Pten functions by inhibiting signaling through the AKT pathway. ${ }^{71 ; 72}$ Although Pten deletion initially led to a transient expansion of hematopoietic stem cell numbers, the hematopoietic stem cell pool in mutant mice become depleted over time. Pten-deficient hematopoietic stem cells engrafted normally in recipient mice, but were unable to sustain multilineage hematopoietic reconstitution over time. ${ }^{71 ; 72}$

Many transcription factors play important roles in regulating hematopoietic stem cell self-renewal. c-Myb can promote the growth of hematopoietic stem cells possibly through the induction of c-myc and the upregulation of c-kit and Flt3. ${ }^{73 ; 74}$ c-Myb deficient mice die at embryonic day 15.5 due to a defect in definitive hematopoiesis. ${ }^{75}$ PcG (polycomb group) genes are involved in the maintenance of cellular memory through epigenetic chromatin modifications. Recent studies have shown that PcG proteins are important in the regulation of hematopoietic stem cell self-renewal, a process in which cellular memory is maintained through cell division. Members of the PRC1 (Polycomb repression complex 1), such as Bmi1(Bone marrow I-1), rae28 and Mel-18, which form DNA-binding protein complexes with gene-suppressing activity, were shown to be involved in lympho-hematopoiesis. ${ }^{76}$ Among these genes, Bmi1 plays an important role in regulating the proliferative activity of stem and progenitor cells. ${ }^{77-79}$ Bmi1 was able to enhance symmetrical expansion of the stem cell pool through self-renewal, induce a marked in vitro expansion of multipotent progenitors, and increase the ability of stem cells to repopulate bone marrow in vivo. ${ }^{80}$ Rae28-deficient hematopoietic stem cells were defective in long-term repopulating ability in serial transplantation experiments. ${ }^{81 ; 82}$ Loss or knockdown of Mel18 leads to increased expression of Hoxb4, and transplanted Mel18deficient bone marrow showed increased hematopoietic stem cell numbers, but a decrease in activity due to arrest in the $\mathrm{G}_{0}$ phase of the cell cycle. ${ }^{83} \mathrm{Gfi1}$ (Growth factor independence 1), a Zinc-finger repressor of transcription, has been recently implicated as a regulator of hematopoietic stem cell self-renewal. It was determined that Gfi1 controls self-renewal by restraining stem cell proliferative potential. ${ }^{84 ; 85} \mathrm{Gfi} 1$-deficient hematopoietic stem cells displayed increased proliferation rates and were functionally compromised in competitive repopulation and serial transplantation assays. Gfi1 might exert these effects by regulating the

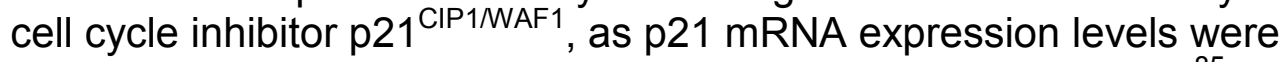
dramatically lower in the Gfi1-deficient hematopoietic stem cells. ${ }^{85}$ p21 ${ }^{\text {CIP1NAF1 }}$ itself was also implicated in the regulation of hematopoietic stem cells. ${ }^{68}$ In its absence, hematopoietic stem cells had impaired serial transplantation capacity. Activation of the Stat5 transcription factor in hematopoietic stem cells led to the dramatic expansion of multipotent progenitors and promoted hematopoietic stem cell self-renewal in vitro. ${ }^{86}$ Deletion of Stat5 resulted in profound defects in 
hematopoiesis and markedly reduced ability of the mutant cells to reconstitute in lethally irradiated mice. ${ }^{87}$ Transduction of adult mouse bone marrow cells with a constitutively activated form of Stat3 increased their regenerative activity in lethally irradiated recipients, whereas the transduction of these cells with a dominant negative form of Stat3 suppressed their regenerative activity. ${ }^{88}$ These studies suggest that Stat proteins play a role in hematopoietic stem cell selfrenewal. The Hox (homeobox) genes encode transcription factors that regulate embryonic body patterning and organogenesis. They play important roles in the regulation of hematopoiesis. Overexpression of HOXB4 in bone marrow cells, which we will discuss in further detail, led to dramatic expansion of hematopoietic stem cells in vivo and in vitro. ${ }^{89-91}$

\subsection{Expanding Hematopoietic Stem Cells for Transplantation}

Understanding the ability of hematopoietic stem cells to replenish themselves and identifying molecules that promote their expansion in vitro is an important clinical goal, since several clinical applications for hematopoietic stem cell expansion could be realized if effective expansion can be achieved. ${ }^{3 ; 91}$ For example, the use of cord blood as a source of hematopoietic stem cells for allogeneic transplantation has been limited by the relatively small number of stem cells in a single harvest. ${ }^{92}$ However, due to their low frequency and the difficulties in culturing stem cells in vitro, effective expansion has not yet been achieved. In vitro culture systems are insufficient to establish the self-renewing capacity of hematopoietic stem cells in general, and often result in differentiation and apoptosis and thus loss of repopulating avtivity. ${ }^{91}$ Thus, new approaches for the in vitro expansion of hematopoietic stem cells are needed. ${ }^{91}$ Although progress has been made in identifying conditions that maintain hematopoietic stem cell activity in culture for a brief period of time, ${ }^{93}$ it has proven difficult to identify combinations of growth factors that cause significant expansion in culture of hematopoietic stem cells. In most cases, combinations of growth factors that can induce proliferation are unable to prevent differentiation of hematopoietic stem cells in long-term cultures. ${ }^{94}$

Assorted signaling molecules and transcription factors have been introduced into hematopoietic stem cells to induce in vitro expansion or immortalization of cells. However, many attempts were unsuccessful because of the inadequate expansion or the unintended induction of leukemias. Ectopic expression of the transcription factor HOXB4 has been shown to dramatically increase the numbers of transplantable hematopoietic stem cells both in vivo and in vitro, and may be the most promising approach. ${ }^{90 ; 95}$

Genetic manipulation of hematopoietic stem and progenitor cells is one important tool for experimental hematology. Successful generation of hematopoietic cells from embryonic stem (ES) cells could provide safe and histocompatible hematopoietic stem cells. ${ }^{96}$ However, achieving stable long-term 
blood engraftment of ES cell-derived hematopoietic stem cells in irradiated mice has proven difficult. To improve the hematopoietic activity of induced hematopoietic cells from ES cells, genetic manipulation was attempted using transcription factors, such as Lhx-2 and GATA-2, or the oncogenic chimeric gene $b c r-a b l$. However, these trials produced limited improvement in ES cell-induced hematopoietic activities. In contrast, enforced HOXB4 expression brought about the best results by promoting the generation of expanded hematopoietic stem cells with reconstitution ability. ${ }^{97}$ Considering the multitude of biological and ethical challenges facing the use of human ES cells at present, expansion of hematopoietic stem cells represents a more realistic and feasible goal. ${ }^{98}$

Four factors (Oct3/4, Sox2, c-Myc, and Klf4) transferred in the mouse oocyte can reprogram mouse embryonic or adult fibroblast cells into pluripotent stem cells that exhibit the essential characteristics of embryonic stem cells. ${ }^{99}$ Subcutaneous transplantation of these induced pluipotent stem (iPS) cells into nude mice resulted in tumors containing a variety of tissues from all three germ layers. Following injection into blastocysts, iPS cells contributed to mouse embryonic development. Generation of hematopoietic stem cells from the iPS cells should be useful in the applications in transplantation.

\subsection{Characteristics of Hox Gene Family Members}

Mammalian Hox genes encode homeodomain transcription factors which play important roles in embryonic body patterning and organogenesis. These genes were initially identified by homology with fruit fly Drosophila Hom genes. ${ }^{100 ; 101}$ In human and mouse, the Hox gene family contains 39 members arranged in 4 distinct clusters (A, B, C, D) on different chromosomes (human 7, 17, 12 and 2; mouse $6,11,15$, and 2 respectively) and 13 paralog groups (Figure 1-3). ${ }^{102-104}$ The $3^{\prime}$ to $5^{\prime}$ chromosomal organization of the genes in each Hox cluster corresponds to their anterior-posterior axis expression pattern in the body, that is, 3' genes affect development of cephalic structures and 5' genes control caudal organs. ${ }^{102}$ Hox genes also show temporal colinearity in expression during development from anterior to posterior direction. The 3' genes are expressed earlier than 5' genes. In general, members of the same homology group are expressed at the same time and have the same anterior boundary of expression. ${ }^{103}$ This orderly activation of Hox genes is also essential for normal hematopoietic development. ${ }^{105}$ The Hox genes we described here belong to the class I group. There is another group of genes other than Hox genes in the homeobox gene family, and they are named as nonclustered or divergent homeobox genes. ${ }^{106 ; 107}$ The divergent homeobox genes are scattered throughout the genome and classified into different small groups based on the similarity in their sequence. In this dissertation, we only focus on the class I, clustered homeobox genes (Hox genes). 


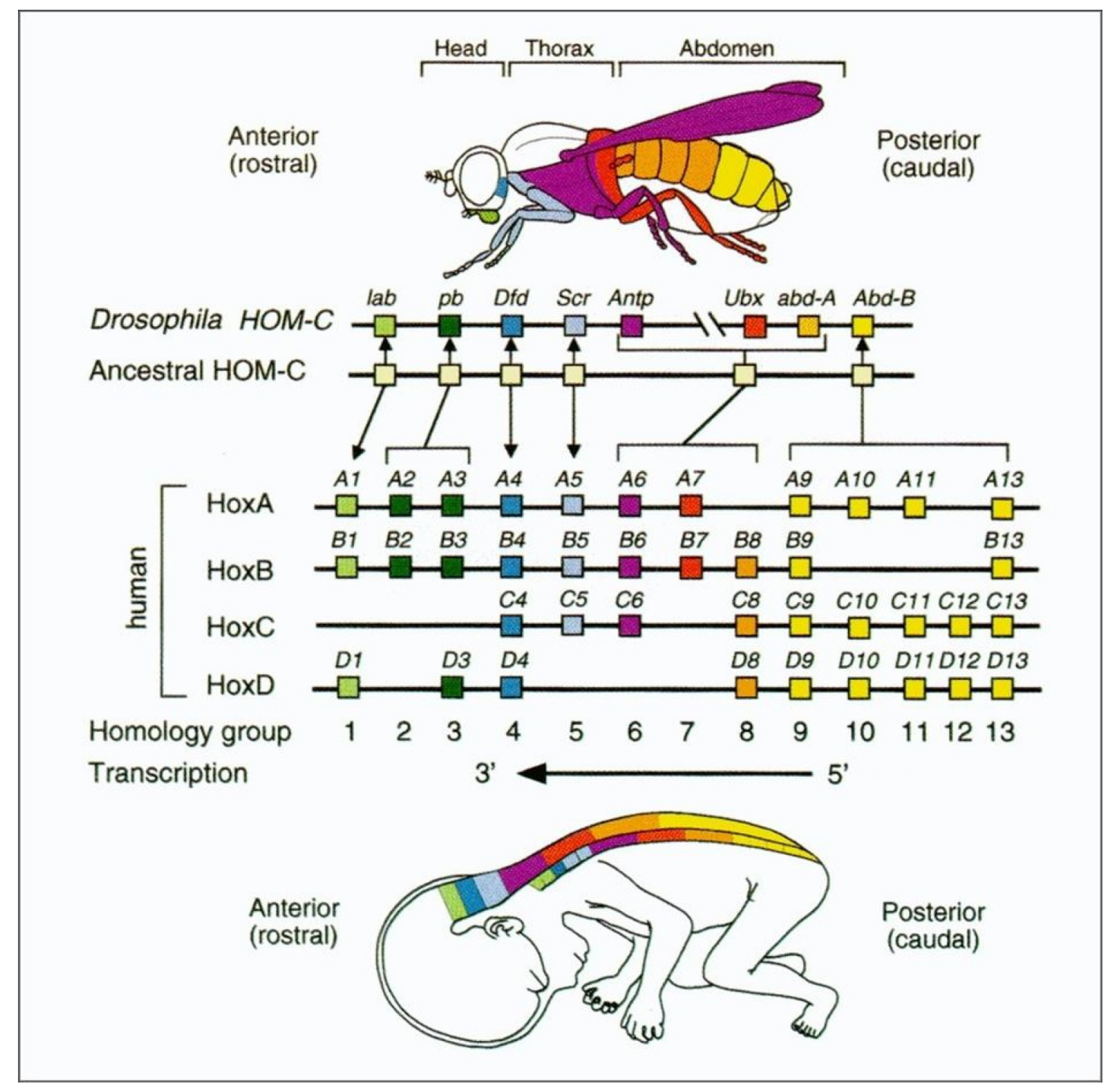

Figure 1-3. Genomic organization and collinear expression patterns of Drosophila Hom genes and mammalian Hox genes.

Reprinted with permission from Pediatric Research. Mark M, Rijli FM, Chambon $P$. Homeobox genes in embryogenesis and pathogenesis. Pediatr.Res. 1997;42:421-429. 


\subsubsection{Functions of Hox Family Members in Hematopoiesis}

The Hox family members of transcription factors are functionally involved in both normal and leukemic hematopoiesis. Genes 3' in the Hox cluster are maximally expressed in hematopoietic stem cells; in contrast, genes 5 ' in the cluster from Hox7 to Hox 11 are maximally expressed in more committed hematopoietic progenitor cells. ${ }^{105}$ Furthermore, expression of Hox7 to Hox11 decreases during differentiation but is maintained in mature blood cells. ${ }^{105}$ Perturbation of orderly Hox gene expression can result in hematologic abnormalities. ${ }^{108}$ Elevated expression of HOX7-11, HOXB6 and HOXB8 has been reported in human acute myeloid malignancies. ${ }^{109-112}$ Disregulation of these Hox proteins in mice also causes hematological abnormalities. ${ }^{13 ; 114}$ Hox genes are also involved in chromosomal translocations seen in human leukemias. For instence, the NUP98 gene at chromosome band 11p15 can undergo chromosomal translocation and be fused to a number of hox family members including HOXA9, HOXA11, HOXA13, HOXC11, HOXC13, HOXD9, HOXD11 and HOXD13 in various hematological malignancies such as myelodysplastic syndromes and acute myeloid leukemia. ${ }^{115-117}$

The functions of several Hox genes, especially those that are highly expressed in immature hematopoietic cells (e.g. Hoxa9, a10, b3 and b4), have been studied extensively. For example, overexpression of Hoxb3 impairs lymphoid development but enhances myeloid development. ${ }^{118} \mathrm{HOXB} 4$ enhances the in vivo repopulating activity of hematopoietic stem cells. ${ }^{95}$ HOXB4 also induces a 40 -fold in vitro expansion of mouse hematopoietic stem cells in cultures containing IL-3, IL-6 and SCF. ${ }^{90}$ HOXB4 enhances hematopoietic stem cell proliferation or the probability of self-renewal, although the gene is not leukemogenic. The genes of trans-paralogs which occupy the same relative location along the $3^{\prime}$ to $5^{\prime}$ axis along the chromosomes are named with same number, and they share a relatively high degree of sequence. ${ }^{104}$ Thus, it is suggested that paralogous genes can act as redundant factors with similar functions. For example, Hoxa3 and Hoxd3 provide equivalent function in gene swapping experiments. ${ }^{119}$ Another example is Hoxa4, Hoxb4, Hoxc4 and Hoxd4. ${ }^{120}$ Overexpression of Hoxc4, a paralog of Hoxb4, also induces in vitro expansion of human immature hematopoietic progenitors. ${ }^{121}$ These findings suggest the importance of the four paralog groups of Hox genes for hematopoietic stem cell expansion. Although there are no obvious hematological abnormalities in mice deficient in Hoxb4 alone, hematopoietic stem cells and progenitor cells deficient in Hoxb3 and Hoxb4 displayed mildly impaired activity both in vitro and in vivo. ${ }^{122}$ 


\subsubsection{Biochemical Characteristics of HOXB4 and Other Hox Family Members}

Mammalian Hox genes usually have relatively small coding sequences with only two exons and a single intron. All contain a highly conserved 60 amino acid common homeobox domain (homeodomain) which contains a helix-turn-helix DNA binding motif encoded by the second exon of Hox genes. ${ }^{111}$ In HOXB4, the homeodomain contains 3 a-helices (Figure 1-4A), among which, helix 3 contacts the major groove of DNA recognizing a conserved "TAAT" core. Helix 1 and 2 do not contact the DNA but stabilize the protein-DNA interaction. The flexible Nterminal domain located just adjacent to helix 1 in the homeodomain extends from the packed helical structure and interacts with the minor groove of DNA to recognize 5' and 3' flanking sequences adjacent to the core consensus sequence. ${ }^{123 ; 124}$ There is a c-terminus acidic domain and a conserved YPWM region upstream of homeodomain in Hox proteins. The YPWM region binds to TALE (three amino acid loop extension) transcription factor family members which act as cofactors of Hox to increase DNA binding specificity and activity of Hox-cofactor complexes. ${ }^{125-128}$

Functional specificity of Hox proteins depends on a number of factors including their specific expression pattern, the particular interacting proteins present in a cell, and the specificity of the particular homeodomain regioin. Due to the high degree of homology within the homeodomain, a core DNA sequence "TAAT" is recognized and bound by a majority of Hox proteins, ${ }^{128}$ raising the question of how the transcriptional and thus functional specificity of these Hox family members is realized. Hox proteins can elicit distinct and variable effects on downstream target genes to regulate variety aspects of developmental fates. Indeed, the DNA binding specificity, affinity and transregulating potential of a specific Hox protein can be modified by the flanking DNA sequence or interaction with cofactors. ${ }^{129-131}$ Hox family members bind to their specific preferred DNA sequence as monomers or as heterdimers with other cofactors (Figure 1-4B), although some deficiencies are thought to exist in monomer binding activity of Hox proteins to DNA. DNA binding affinity of Hox proteins can also be regulated by posttranslational modifications. For example, PKC (protein kinase C) dependent serine and threonine phosphorylation can decrease the binding affinity of Hoxa9 to its specific DNA consensus sequence. ${ }^{132}$ Phosphorylation of conserved homeodomain tyrosine residues decreases the DNA binding ability of Hoxa10 to some DNA sequences, but increases the binding affinity of Hoxa9 to other DNA sites. ${ }^{133 ; 134}$ Hox proteins form heterdimers with Pbx or Meis members of the TALE family of transcription factors (Figure $1-4 \mathrm{~B}$ ). ${ }^{135}$ Hox homologs 1 to 10 can cooperatively bind to DNA through interaction with Pbx in the core YPWM residues 5' upstream of homeodomain of HOXB4. Hox homologs 9 to 13 can directly interact with Meis. ${ }^{136}$ Hox homologs 9 and 10 can interact with both Pbx and Meis, and functional trimeric interactions between them have been reported. ${ }^{137}$ Interaction with these TALE family cofactors is hypothesized to increase the DNA binding ability of some Hox family members to their target 
A

Homeodomain-conserved for DNA binding

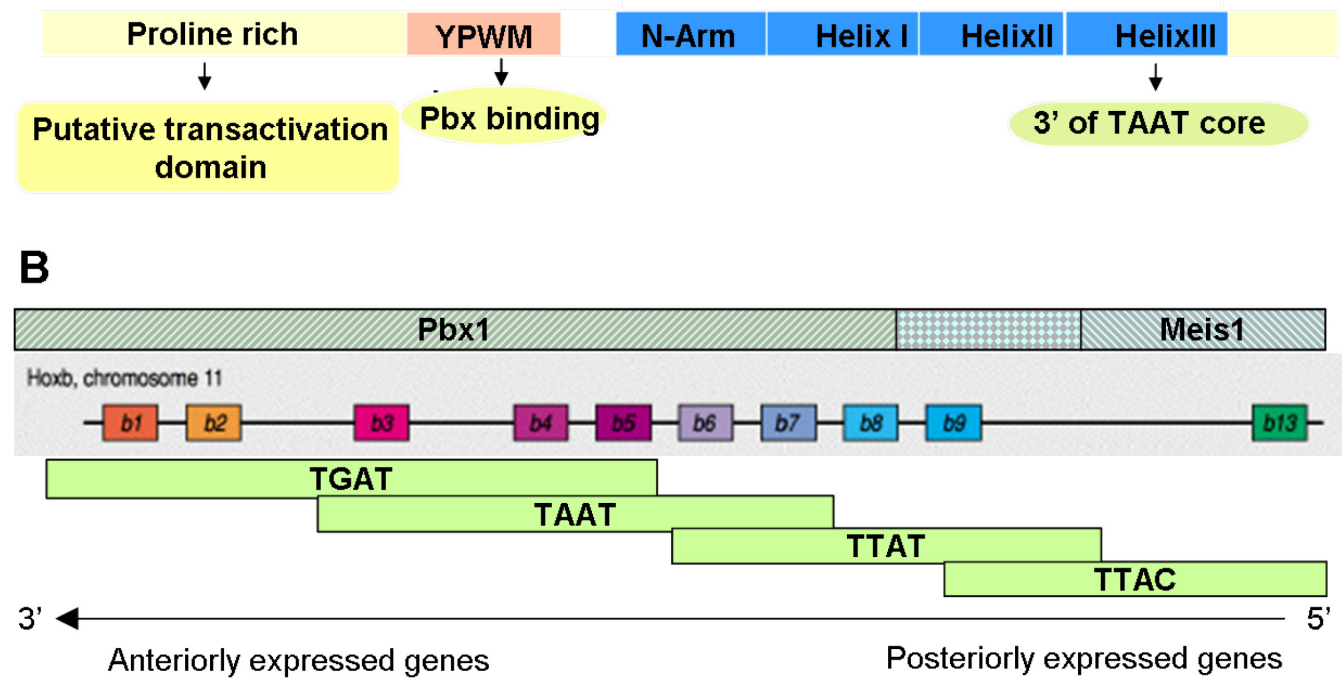

Figure 1-4. Biochemical characteristics of Hox proteins. (A) Functional domains of HOXB4. (B) Cofactors and DNA binding specificity of Hox family members. 
genes. ${ }^{138}$ In addition, the transcriptional specificity of Hox proteins also lies in their ability to act both as transcriptional activators and repressors in different conditions. The complexity of Hox regulation of downstream target genes is underscored by the observation that they can regulate different target genes either dependent or independent of TALE family cofactors. ${ }^{133 ; 139}$

It is known that HOXB4 regulates hematopoietic stem and progenitor cell expansion through a transcription-dependent mechanism. ${ }^{140}$ A HOXB4 mutant incompetent for DNA binding failed to cause the proliferation of transduced bone marrow in vitro and hematopoietic stem cell expansion in vivo. ${ }^{140}$ Similar to other Hox family members, HOXB4 contains a homeodomain that is required for DNA binding. In this homeodomain, the helix 3 region is essential for recognizing and interacting with the consensus DNA regulatory element containing "TAAT" or "ATTA" core sequence in the promoters of Hox target genes. In vitro studies have proven that HOXB4 has preference for specific flanking nucleotides (Figure 1-5). ${ }^{129}$ HOXB4 can cooperatively bind to 5' TGATTAAT 3' sequences with its cofactor Pbx through its "YPWN" residues. However, it was demonstrated that a HOXB4 mutant that cannot bind $\mathrm{Pbx}$ was functional and caused in vitro proliferation and elevated in vivo regenerative potential in bone marrow cells. ${ }^{140}$ The proline-rich $\mathrm{N}$-terminus region of HOXB4 is conserved in other proteins such as p53, AP2, HOXD4 and HOXA13, in which it functions as a transactivation domain.

\subsubsection{Hox Downstream Target Genes}

Hox transcription factor family members can act as key masters for controlling cellular identity and play critical roles in controlling embryogenesis and organ development. Although much is now known about their regulation and function, very little is known of their effector genes. Recent studies have focused on identifying their common or unique downstream target genes to facilitate understanding of their functions, especially in the process of normal and malignant hematopoiesis. Such understanding may provide means for hematopoietic stem cell engineering approaches to hematopoietic. For the purpose of identifying downstream target genes, various approaches have been taken including high throughput genetic screens, in which, the gene expression profiles of cells overexpressing specific Hox proteins are analyzed.

A small number of Hox target genes have been identified in different biological systems that play roles in cellular identity and function. However, the experimental procedures used for identification of each target gene sometimes make it difficult to determine whether they are truly direct targets. For instance, for some direct targets, the DNA sites bound by Hox factors have not been confirmed experimentally. A summary of identified Hox target genes which are involved in a variety of cellular processes have been reported. ${ }^{141}$ In the list outlined in this review, ${ }^{141}$ the identified target genes of Hox proteins participate in 
Nucleotides preferred $5^{\prime}$

A, G $>$ C, T $\quad$ C $>$ G, T $>$ A
Nucleotide preferred 3'

TAAT $\quad$ T, G $>$ A $>$ C $\quad$ G, A $>$ T, C

Figure 1-5. HOXB4 has preference for the nucleotides flanking the "TAAT" core binding sequence.

Source: Pellerin I, Schnabel C, Catron KM, Abate C. Hox proteins have different affinity for a consensus DNA site that correlate with the positions of their genes on the hox cluster. Mol.Cell Biol. 1994;14:4532-4545. 
organogenesis and cellular differentiation, cell adhesion and migration, cell cycle and apoptotic pathways.

The TALE class homeodomain transcription factor Irx5 and the small GTPase Rap1 were reported to be regulated by Hoxb4 in development. ${ }^{12 ; 143}$ However, no specific target genes of HOXB4 have been identified in hematopoietic cells untill recently. There is only one report that addresses the targets of HOXB4 in hematopoietic cells. ${ }^{144}$ In this report, the downstream pathways ectopically expressed HOXB4 were identified in adult and embryo-derived hematopoietic stem cells. Gene expression profiling assays were performed in combination with functional assays using enriched adult hematopoietic stem cells and embryonic derivatives overexpressing an inducible form of HOXB4. A set of overlapping genetic changes was generated from these systems and selected to represent common targets of HOXB4 in embryonic and adult hematopoietic stem cells. ${ }^{144}$ In functional assays, HOXB4 activity was found to protect adult hematopoietic stem cells from the detrimental effects of the proinflammatory cytokine TNF- $\alpha$, which likely contributes to the competitive repopulation advantage of HOXB4overexpressing cells in vivo. ${ }^{144}$ It was also demonstrated that HOXB4 activity and FGF signaling were intertwined, since HOXB4-mediated expansion of adult and ES cell-derived hematopoietic stem cells was enhanced by inhibition of FGF receptors. ${ }^{144}$ In this report, however, no evidence was provided to prove that any of the target genes was a direct transcriptional target of HOXB4. Instead, their data suggest that multiple pathways downstream of HOXB4 could be primary or secondary changes related to HOXB4 overexpression.

\subsection{Role of HOXB4 in Hematopoietic Stem and Progenitor Cell Expansion}

Hematopoietic stem cells or repopulating cells are able to self-renew and differentiate into cells of all hematopoietic lineages, and because of this unique property, hematopoietic stem cells have been used for stem cell transplantation and gene therapy applications. However, the inability to expand hematopoietic stem cells has been a significant limitation for clinical applications. To date, the most successful hematopoietic stem cell expansion method involves the overexpression of the HOXB4 gene. ${ }^{145 ; 146}$

\subsubsection{Overexpression of HOXB4 In Vivo}

Retroviral-mediated overexpression of HOXB4 enhances hematopoietic repopulation, through the increased self-renewal of hematopoietic stem cells. ${ }^{90 ; 95 ; 147}$ Serial transplantation studies revealed a greatly enhanced ability of HOXB4-transduced bone marrow cells to regenerate the most primitive hematopoietic stem cell compartment, resulting in $\mathbf{5 0}$ fold higher numbers of transplantable totipotent hematopoietic stem cells in primary and secondary recipients (Figure 1-6) ${ }^{95 ; 145}$ After transplantation into primary recipient mice, 


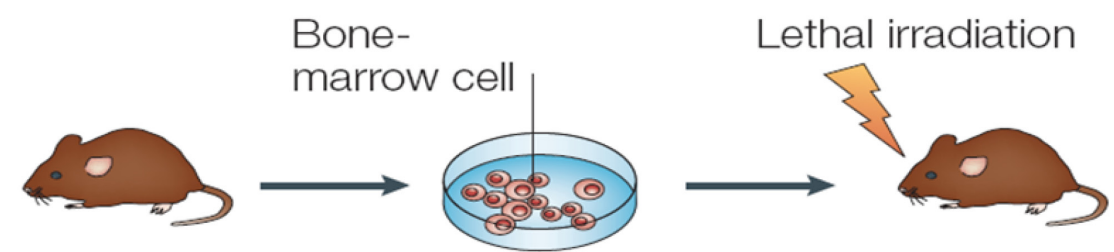

Untransplanted

In vitro culture with

Primary donor a vector expressing Hoxb4 or neor

transplant recipient

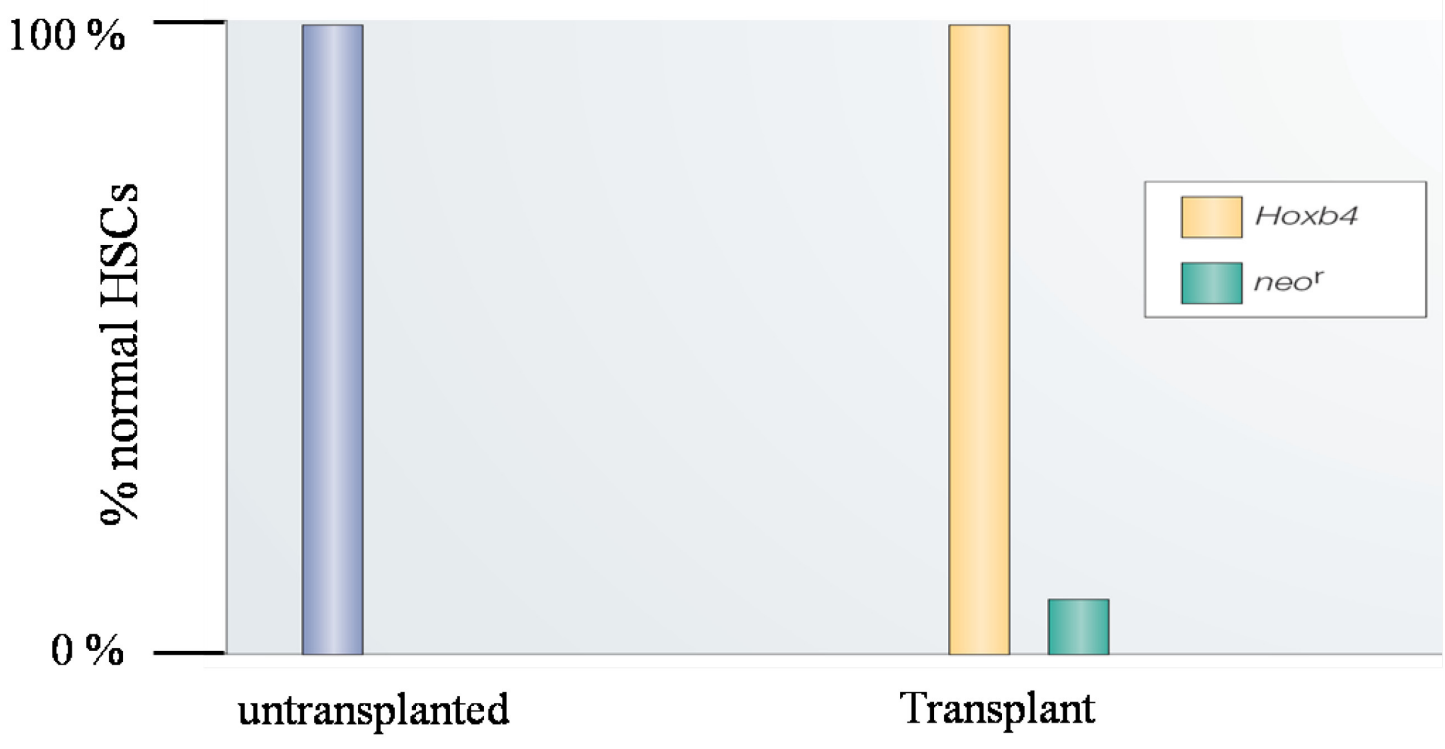

Figure 1-6. HOXB4 induces expansion of hematopoietic stem cells in vivo.

Reprinted with permission from Nature Publishing Group. Sorrentino BP. Clinical strategies for expansion of haematopoietic stem cells. Nat.Rev.Immunol. 2004;4:878-888. 
there was a rapid regeneration to normal pretransplantation levels in the numbers of hematopoietic progenitors and mature blood cells in the mice transplanted with control vector-transduced bone marrow cells, whereas hematopoietic stem cell numbers recovered to only $5 \%$ to $10 \%$ of normal levels. This finding demonstrates that hematopoietic stem cells were significantly restricted in their self-renewal activity during the process of hematopoietic repopulation (Figure 1-6) ${ }^{147}$ In contrast, HOXB4 vector-transduced cells were capable of fully reconstituting the stem cell compartment, and resulted in a 14 fold more hematopoietic stem cells than the control-transduced bone marrow cells in primary transplant recipients. Furthermore, HOXB4-overexpressing hematopoietic stem cells did not expand above the normal level observed in unmanipulated mice, indicating that its overexpression did not override the regulatory mechanisms that maintain normal hematopoietic stem cell pool size. $^{147}$

In addition, recovery of stem cell numbers in HOXB4 recipients was reported to be extremely rapid, reaching $25 \%$ of normal by 14 days posttransplant, some 80 fold greater than that seen in control transplanted recipients. ${ }^{89}$ The heightened in vivo expansion of HOXB4-overexpressing hematopoietic stem cells was not associated with identifiable anomalies in the peripheral blood of the transplanted mice. ${ }^{95 ; 145 ; 146}$ In a clinically relevant nonhuman primate model, HOXB4 overexpression in $\mathrm{CD} 34^{+}$cells was shown to dramatically promote expansion and engraftment of short-term repopulating cells and a significant, but less pronounced, expansion of long-term repopulating cells. ${ }^{148}$

\subsubsection{Overexpression of HOXB4 In Vitro}

In addition to its function in promoting stem cell expansion in vivo, HOXB4overexpression also confers stem cell expansion in bone marrow cell cultured in vitro. Cultures of HOXB4 -transduced bone marrow cells resulted in rapid, extensive, and highly polyclonal hematopoietic stem cell expansion, with over 1000 fold greater numbers of stem cells relative to control vector-transduced cells, which instead undersewnt stem cell loss during a 14 day culture period with a corresponding 40 fold net stem cell decrease relative to input levels (Figure 1-7).${ }^{90}$ Importantly, those in vitro expanded cells retained full lympho-myeloid repopulating ability and enhanced regenerative potential in vivo, indicating the feasibility of achieving in vitro expansion of hematopoietic stem cells without functional impairment in vivo. ${ }^{90}$ Enhanced proliferation of day 12 CFU-S and immature mixed clonogenic progenitors in vitro was also documented in HOXB4overexpressing bone marrow cells. ${ }^{95 ; 149}$

The cellular effects and mechanisms of HOXB4-induced cell expansion were studied in vitro. HOXB4-overexpressing bulk bone marrow cells were reported to show rapid dominance in mixed culture and shortened population doubling time in vitro. ${ }^{89} \mathrm{HOXB} 4$ overexpression in vitro resulted in a stabilization of the 


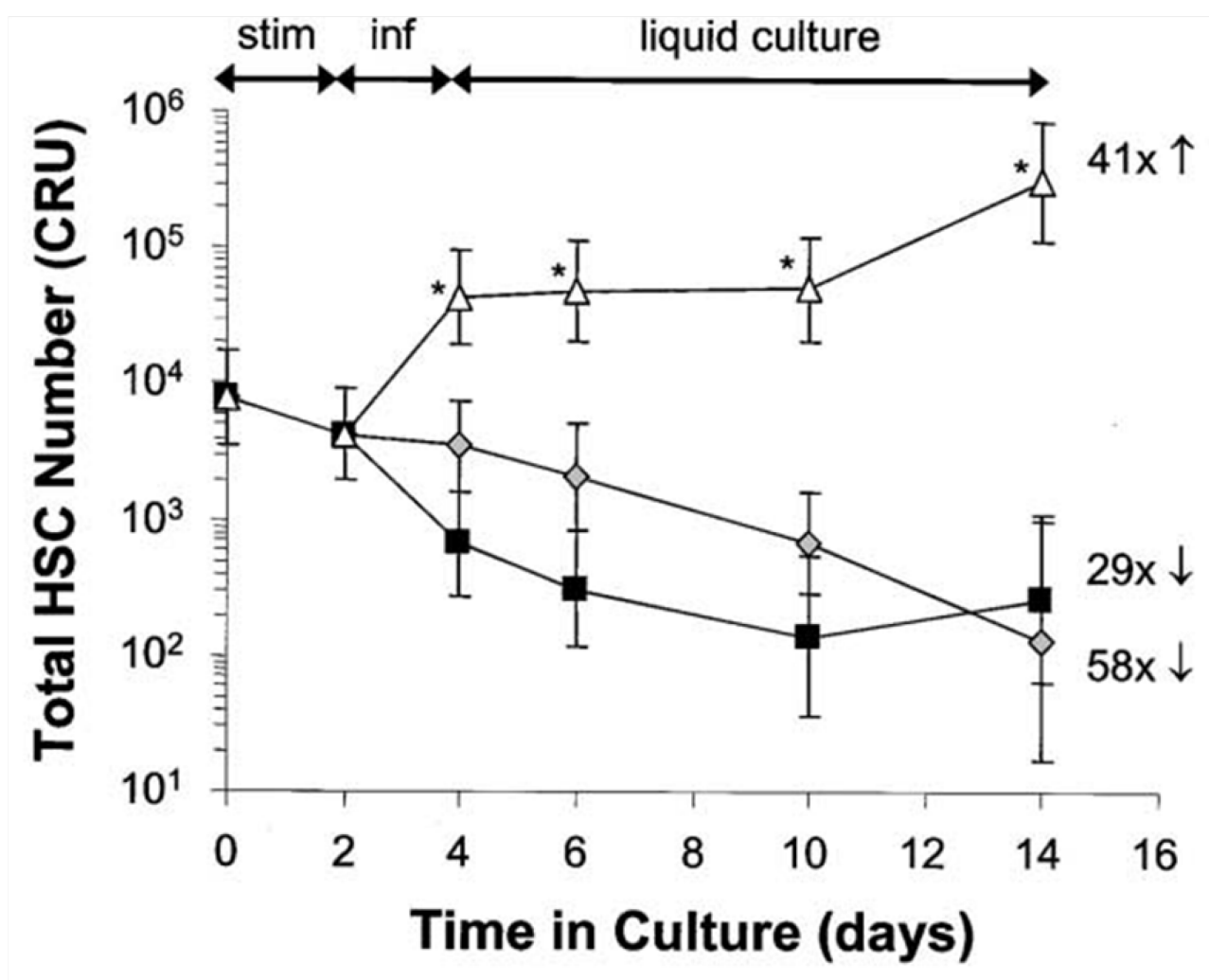

Figure 1-7. HOXB4 induces expansion of hematopoietic stem cells in vitro.

Reprinted with permission from Elsevier. Antonchuk J, Sauvageau G, Humphries RK. HOXB4-induced expansion of adult hematopoietic stem cells in vitro. Cell 2002;109:39-45. 
proliferation rate, while the proliferation rate of control-transduced bone marrow cultures gradually declined. ${ }^{150}$ In addition, HOXB4-overexpressing bone marrow cells displayed a more homogenous morphology and contained increased numbers of hematopoietic progenitor cells capable of generating primitive colonies in vitro. In contrast, control-transduced bone marrow cells in long-term cultures showed hallmarks of myeloid differentiation and a reduced secondary colony forming activity. ${ }^{150}$

\subsubsection{ES Cell Specification}

HOXB4 also plays a role in the specification of hematopoiesis at the earliest stages of development by enhancing the hematopoietic differentiation potential of ES cells. ${ }^{146 ; 151}$ Overexpression of HOXB4 in murine ES cell-derived primitive progenitors combined with culture on hematopoietic stroma cells induced a switch to the definitive hematopoietic stem cell phenotype. ${ }^{146}$ The induction of differentiation was initiated with EB (embryoid body) formation, and subsequent differentiation and maintenance of hematopoietic cells was achieved by coculture with OP9 cells. ${ }^{146}$ The transient overexpression of HOXB4 for only several weeks in hematopoietic progenitor cells initiated conversion of progenitor cells to immature transplantable stem cells. ${ }^{146}$ Leukocytes derived from ES cells ectopically expressing HOXB4 were immunologically functional, implying the potential use of ES cell-derived hematopoietic progenitor cells in the treatment of hematologic and immunologic diseases. ${ }^{152}$ These results provide new evidence implicating HOXB4 in the control of very early development of the hematopoietic system.

\subsubsection{Potential Toxicity of HOXB4 Overexpression}

Ectopic HOXB4 expression was reported to have a concentration-dependent effect on lineage differentiation in mouse and human hematopoietic cells. The existence of threshold levels were suggested for HOXB4 activity that control the differentiation and self-renewal behavior of hematopoietic stem and progenitor cells. ${ }^{153}$ Enforced HOXB4 expression caused a significant reduction of erythroid and myeloid progenitors and $\mathrm{B}$ lymphoid compartment in vitro and in vivo when using a strong viral promoter for expression. ${ }^{154}$ Transducing CD $34^{+}$cells from large animals including nonhuman primates, dogs, and humans with HOXB4retroviral vectors can cause increased colony formation ability in dog cells compared to human peripheral blood, human cord blood, and baboon cells. ${ }^{155}$ High levels of HOXB4 overexpression resulted in immortalization of primitive hematopoietic cells from mice and dogs; in contrast, the cells from monkeys and humans were not immortalized due to the relative low expression level of HOXB4. ${ }^{155}$ In differentiated ES cells ectopically expressing HOXB4 in vitro, myeloid development can be enforced and T lymphoid development can be suppressed over a wide range of expression levels of HOXB4. ${ }^{156}$ These findings 
suggest that the growth promoting effects of HOXB4 are critically dependent on its expression levels, which can result in important species-specific differences in potency. Based on these facts, the therapeutic window of HOXB4 expression levels must be carefully defined before initializing clinical trials.

Retroviral vector-mediated insertional mutagenesis can occur with HOXB4 overexpression. Hematopoietic stem cell gene therapy has been used to treat patients with a number of life-threatening diseases. However, some patients have developed retroviral vector-mediated leukemia after treatment. ${ }^{157}$ Myeloid leukemia was reported to occur in large animals (dogs and macaques) 2 years after transplantation with HOXB4-overexpressing cells, but not in control retroviral vector transplanted large animals. ${ }^{157}$ However, based on the activation of nearby genes known to be involved in leukemogenesis, the integration sites observed in the leukemic clones containing HOXB4 were very likely a combination consequence of sustained in vivo proliferation through the activated ongenes and expansion effects by HOXB4. ${ }^{157}$

When using short hairpin RNAs to knockdown HOXB4 expression in a cell line from the leukemic cells, profound growth inhibition and cell death occurred with downregulation of the HOXB4 transgene. This evidence indicates that HOXB4 overexpression using retroviral vectors can pose a significant risk of leukemogenesis in large animals. Thus, safety issues related to HOXB4mediated gene therapy must be considered. Newer vectors based on lentivirus are now available that may circumvent this limitation. ${ }^{158}$

\subsubsection{Hoxb4 Knockout and Other Hox-Deficient Mice}

As overexpression of HOXB4 leads to dramatic hematopoietic phenotypes, it is a surprise that $\mathrm{Hoxb}^{-/-}$mice show only a defect in mild hematopoietic stem cell proliferation. ${ }^{159}$ In Hoxb4 ${ }^{-/}$mice, the cellularity in spleen and bone marrow, red blood cell counts, and hemoglobin values were minimally reduced. However, the hematopoietic lineage distribution was normal and in bone marrow and fetal liver, only a mild reduction of hematopoietic stem and progenitor cell numbers were found. ${ }^{159}$ The conclusion from this study was that Hoxb4 is not required for generation of hematopoietic stem cells and steady state maintenance of hematopoiesis. ${ }^{159} \mathrm{c}$-Kit ${ }^{+}$fetal liver cells from Hoxb $4^{-/}$mice expressed higher levels of several other Hoxb cluster genes than control wild type cells by quantitative PCR assay, indicating that normal hematopoietic stem cell activity in $H o x b 4^{-/}$mice might be due to a compensatory upregulation of other Hox genes. In order to determine whether this hypothesis is true, the entire Hoxb cluster was deleted. ${ }^{160}$ However, again, this did not lead to major defects in hematopoiesis. ${ }^{160}$ Hoxb1-b $9^{-/}$fetal liver cells retained full competitive repopulation potential and the ability to regenerate all myeloid and lymphoid

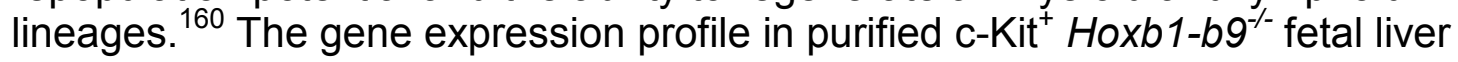
cells revealed expression changes in Hoxa4, a11, and c4. Primitive 
C-kit ${ }^{+}$Scal $1^{+} \mathrm{Lin}^{-}$hematopoietic stem and progenitor cells lacking Hoxb3 and Hoxb4 also displayed impaired proliferative capacity in vitro and in vivo. ${ }^{122}$ As no defects in homing were observed, a slower regeneration of mutant hematopoietic stem cells was suggested. Furthermore, treatment with cytostatic drugs demonstrated slower cell cycle kinetics of hematopoietic stem cells deficient in Hoxb3 and Hoxb4, resulting in increased tolerance to antimitotic drugs.

In the Hoxa9 $9^{--} ; b^{--} ; b 4^{-/}$triple deficient mice, the reconstitution defect in hoxa9 deficient hematopoietic stem cells was not enhanced further in the triple deficient cells. Therefore, none of these hox proteins was proved to play absolutely essential role for generation or maintenance of all major blood lineages. ${ }^{161}$ Some other Hox knockout mice have been reported to show defects in embryonic development. Hoxa $4^{-/-}$or Hoxa ${ }^{-/-}$mice showed homeotic transformation of cervical vertebrae in development. However, defects were not observed in other tissues that normally express these genes. ${ }^{162}$ Hoxc4 is a mouse homeobox gene located at the $3^{\prime}$ end of the HoxC cluster. Of the Hoxc genes, Hoxc4 is expressed in the most anterior regions of the central nervous system and prevertebral column. Hoxc $4^{-/}$mice were viable and fertile, and various abnormalities in the cervical and thoracic regions were documented. ${ }^{163}$ However, no studies have been performed on the hematopoietic system yet. Similarly, Hoxd $4^{-/-}$mice also had defects in skeleton development with homeotic transformations of the second cervical vertebrae (C2) to the first cervical vertebrae $(\mathrm{C} 1)$ and malformations of the neural arches of $\mathrm{C} 1$ to $\mathrm{C} 3$ and of the basioccipital bone. ${ }^{164}$ These phenotypic similarities and differences between Hoxd4 and previously reported Hoxa4, Hoxb4 and Hoxc4 deficient mice suggest that Hox gene paralogs have both redundant and unique functions.

$\mathrm{Pbx} 1$, described above as a cofactor of Hox, is a homeodomain transcription factor that was originally identified as the product of a proto-oncogene in acute pre-B-cell leukemia, and is also a global regulator of embryonic development. $\mathrm{Pbx} 1$ and a subset of homeodomain proteins collaboratively bind to DNA as molecular complexes with potential consequences for mammalian development. Due to embryonic lethality in its absence, caused by severe hypoplasia or aplasia of multiple organs and widespread patterning defects of the axial and appendicular skeleton, ${ }^{165 ; 166} \mathrm{~Pb} \times 1^{-/-}$embryonic stem cells were studied instead and failed to generate common lymphoid progenitors, resulting in a complete lack of B and NK cells, and a partial impairment of T-cell development in transplanted mice. ${ }^{165} \mathrm{~B}$-cell development was rescued from common lymphoid progenitors following restoration of $\mathrm{Pbx} 1$ expression in its deficient ES cells. This critical functions of $\mathrm{Pbx} 1$ occured at a stage between hematopoietic stem cell development and $\mathrm{B}$ cell commitment as one earliest-acting transcription factor to regulate de novo $B$ lineage lymphopoiesis. ${ }^{165}$

With the information generated from Hox deficient mice, it seems like no single Hox gene plays an essential physiological role in hematopoiesis maintenance or hematopoietic stem cell generation. Instead, a redundant network of genetic 
interactions between different Hox genes in primitive hematopoietic cells is most likely. It was recently reported that Hoxb4 and its other three paralogs: Hoxa4, Hoxc4 and Hoxd4 had the similar effect in promoting hematopoietic stem and progenitor cells expansion in vitro. ${ }^{167}$ Our lab also demonstrated that when overexpressed in bone marrow cells, HOXA4, B4, C4 and D4 showed similar effect in promoting engraftment in the transplanted mice (Figure 1-8). This redundancy may explain the mild defect phenotype in $\mathrm{Hoxb}^{-/-}$mice, ${ }^{158}$ and suggests that Hox4 paralogs may be required in hematopoiesis and hematopoietic stem cell functions.

\subsubsection{Mechanisms of HOXB4 Function}

Although it is well-known that enforced expression of HOXB4 can induce stem cell self-renewal and expansion. Relatively little is know about the molecular mechanisms by which this occurs. Key domains in the HOXB4 protein were mutated to investigate their roles in protein function and stem cell expansion. ${ }^{140}$ The in vitro and in vivo expansion capacity of DNA-binding HOXB4 mutants was completely lost, indicating that HOXB4 must bind DNA in order to promote selfrenewal. ${ }^{140}$ In contrast, a point mutation in "YPWM" motif, which disrupts Pbx cofactor binding, still conferred a pronounced in vitro expansion advantage in transduced bone marrow populations, and those cells also showed dramatically enhanced regenerative potential in vivo. ${ }^{140}$ This finding suggests that the capacity of HOXB4 to induce hematopoietic stem and progenitor cell expansion is DNA-binding dependent but does not require direct $\mathrm{Hox}$ and $\mathrm{Pbx}$ interaction. In another report, when $\mathrm{Pbx}$ expression was knocked down in HOXB4 overexpressing bone marrow cells, the hematopoietic stem cell generation ability was $>20$ times more competitive than the wild type cells overexpressing HOXB4. ${ }^{168}$ Furthermore, the myeloid and lymphoid lineage differentiation activity and the hematopoietic stem cell pool were normal in vivo in mice transplanted with $P b x$ knocked down cells. ${ }^{168}$ This result suggests the existence of a distinct mechanism that limits the expansion of HOXB4 transduced hematopoietic stem cells in mice. ${ }^{168 ; 169}$

The specific mechanisms by which HOXB4 promotes hematopoietic stem cell expansion are still a mystery. No direct targets of HOXB4 have been defined in vivo or even in primary cells so far. Although as discussed above, the TNF- $\alpha$ pathway was recently shown to be downstream of HOXB4, using gene expression profiling in long-term cultured bone marrow cells. However, it is unclear whether any of the genes on this pathway are direct transcriptional targets of HOXB4. ${ }^{144}$ 


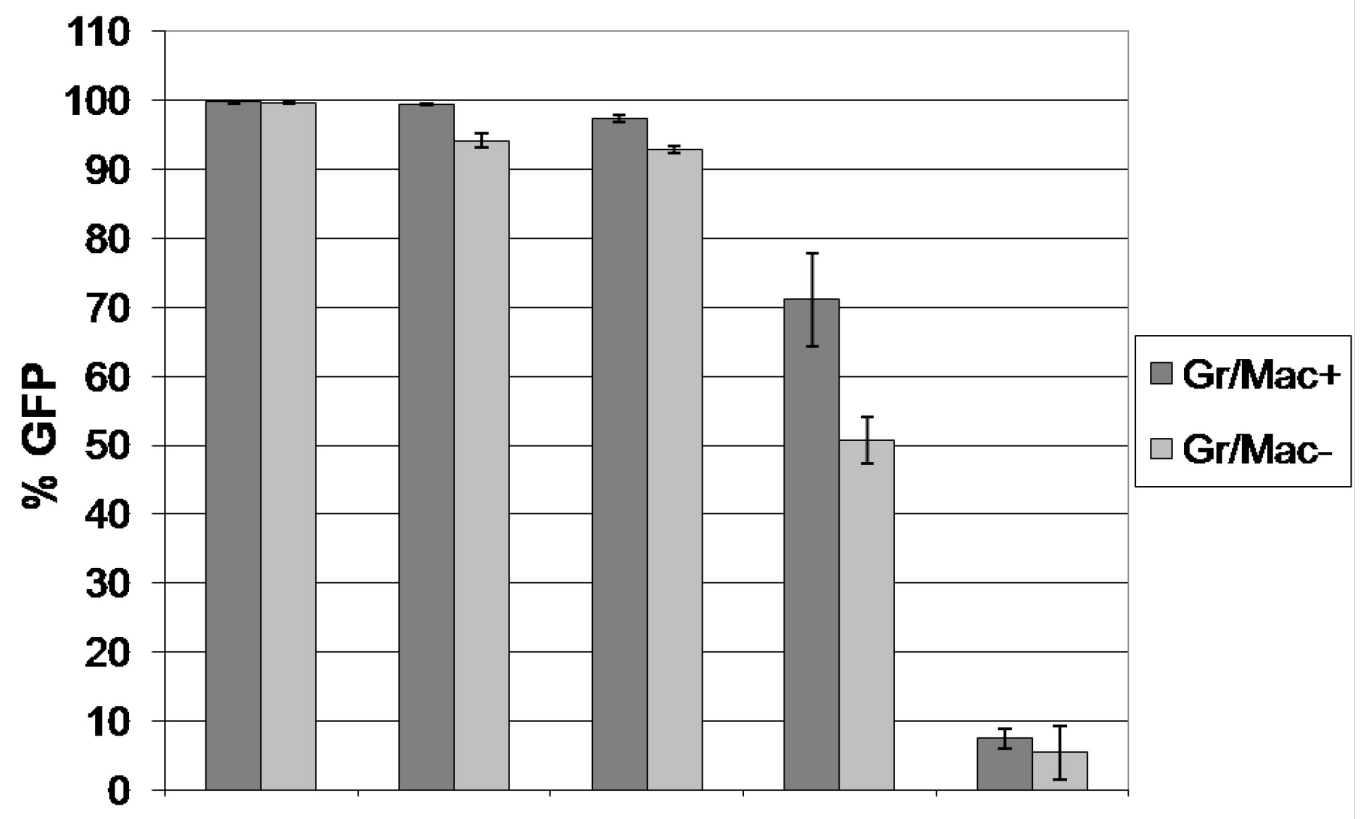

HOXA4 HOXB4 HOXC4 HOXD4 GFP

Figure 1-8. Redundancy of HOX4 paralogs in promoting hematopoietic stem and progenitor cell expansion. Flow cytometry-detected GFP percentage of the peripheral blood cells from 12 weeks-transplanted mice with bone marrow cells transduced by MSCV-HOX4-IRES-GFP or MSCV-IRES-GFP control vectors. Gr and Mac: markers for rapid turnover myeloid cells.

Source: Unpublished data receieved from Brian.P. Sorrentino's lab on October 1st, 2009. 


\subsection{Hemgn}

\subsubsection{Expression Pattern and Biochemical Characteristics of Hemgn}

In my study, I have focused on a new target gene of HOXB4 in hematopoietic cells called Hemogen (Hemgn) or EDAG. Hemgn is a recently discovered nuclear protein and expresses in testis and hematopoietic tissues; specifically, it is highly expressed in hematopoietic stem and progenitor cells and is downregulated as hematopoietic cells differentiate. ${ }^{170}$ The expression of Hemgn during embryogenesis shows a spatial and temporal pattern corresponding to the ontogeny of hematopoiesis. The expression switches from yolk sac blood islands, to fetal liver hematopoietic cells during embryogenesis to bone marrow and spleen in adult mice. ${ }^{170}$ In adult mouse tissues, Hemgn mRNA expression is restricted to spleen and bone marrow cells (Figure 1-9A). In bone marrow cells, relatively high levels of Hemgn mRNA were detected in $\mathrm{Lin}^{10} \mathrm{C}-\mathrm{kit}^{+} \mathrm{Scal} 1^{+}, \mathrm{CD} 34^{+}$, and Lin $^{-}$hematopoietic stem and progenitor cell populations (Figure 1-9B). ${ }^{170}$ In adult mouse spleen, Hemgn was expressed specifically in the erythrocytes red pulp regions but not in the white pulp which contains $B$ and $T$ lymphocytes (Figure 1-9C). ${ }^{170}$ Immunostaining studies showed that Hemgn was localized in nuclei and absent in the cytoplasm (Figure 1-9D). ${ }^{170}$ Moreover, hemgn is also expressed in testis in a variant splice form using an alternative promoter, suggesting a complex transcriptional regulation. ${ }^{171}$

A human homolog of Hemgn, HEMGN, also called EDAG, maps to chromosome 9q22, which contains a cluster of important genes involved in hematopoiesis. The expression pattern of EDAG is similar to its mouse homolog. ${ }^{172}$ Like Hemgn, EDAG exhibited specific expression in human hematopoietic cells in the adult bone marrow and fetal liver. No EDAG transcripts were detected in peripheral blood cells or other non-hematopoietic tissues, such as adult liver, heart, brain, skeletal muscle, kidney, spleen, pancreas, tonsil, and colon. ${ }^{172}$

Sequence analysis shows that Hemgn and EDAG contain a nuclear localization motif and a coiled-coil domain, but no other recognizable protein motifs. At the nucleotide level, human and mouse Hemgn homologs show $43 \%$ identity, although the sequence conservation between the nuclear localization signal and the coiled-coil domains are higher. Coiled-coil domains of a protein are protein-protein interaction domains containing multiple $\alpha$-helices twisting around each other to form a supercoil. A protein containing coiled-coil domains can usually interact with other coiled-coil proteins for protein-protein recognition and interaction.

Based on the nuclear localization of EDAG, the role of EDAG as a transcription factor has been investigated. ${ }^{173}$ Using an yeast and mammalian two-hybrid system, it was suggested that that EDAG has transactivation 
A

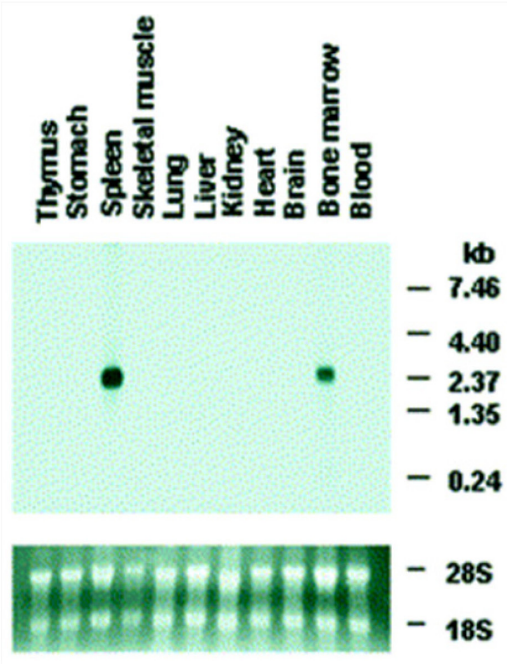

C

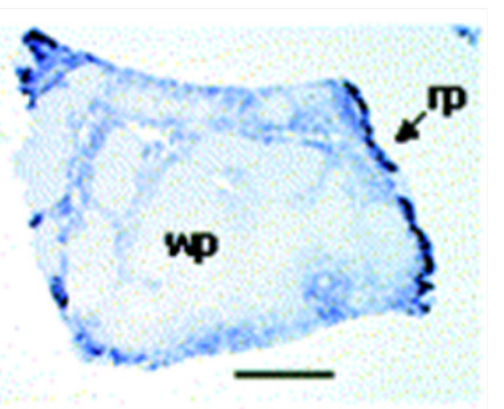

B
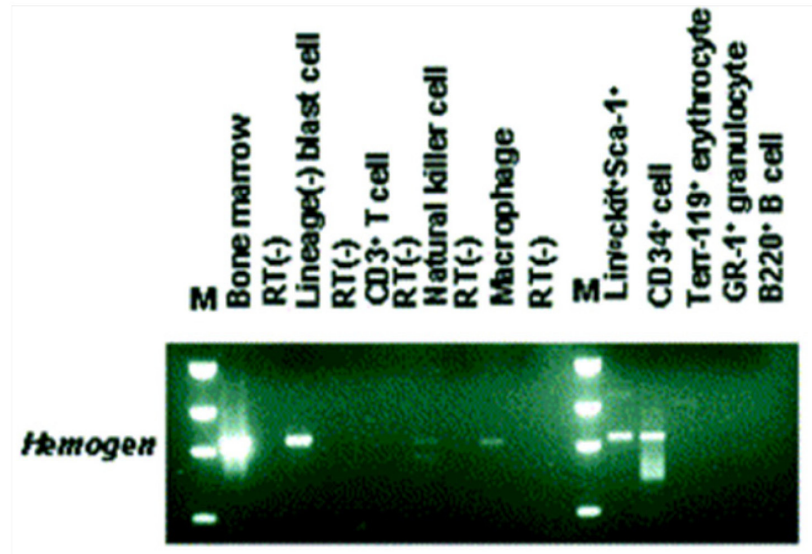

Histone

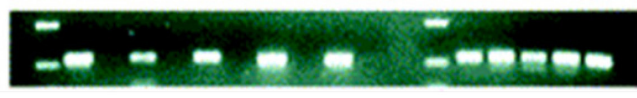

D

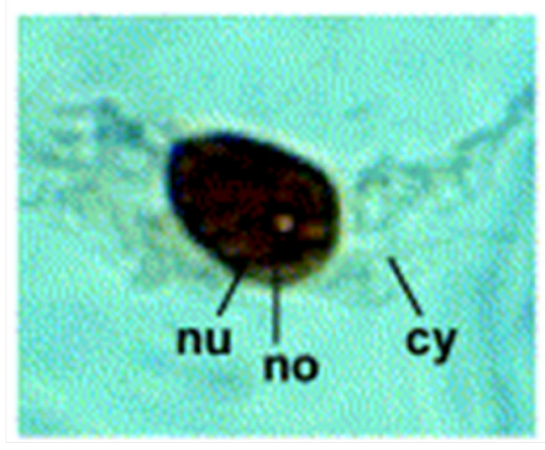

Figure 1-9. Expression and localization of Hemgn. (A) Northern blotting for mRNA of Hemgn in indicated tissue. A $2.4 \mathrm{~kb}$ message was detected in the spleen and bone marrow. (B) RT-PCR detected mRNA expression of Hemgn in indicated tissue. (C) By hybridization with antisense RNA probe, Hemgn transcripts were localized in the red pulp (rp) but not in the white pulp (wp) in mouse spleen. (D) The Hemgn-FLAG fusion protein was transfected into COS-7 cells and detected with anti-FLAG antibody. The signal was localized in the cell nuclei (nu) but not in the nucleoli (no) or cytoplasm (cy).

Reprinted with permission from Elsevier. Yang LV, Nicholson RH, Kaplan J, Galy A, Li L. Hemogen is a novel nuclear factor specifically expressed in mouse hematopoietic development and its human homologue EDAG maps to chromosome 9q22, a region containing breakpoints of hematological neoplasms. Mech.Dev. 2001;104:105-111. 
activity, indicating that EDAG could be a transcriptional regulator. ${ }^{173}$ EDAG cDNA fused with the Gal4 DNA-binding domain (DBD) was transformed into yeast cells and found to transcriptionally activate a chromosomally integrated lacZ reporter gene. This result was also confirmed in mammalian cells through luciferase assay.

\subsubsection{Functions of Hemgn}

EDAG expression is very high in leukemia cell lines such as K562, HEL and Mo7e and megakaryoblast leukemia cell lines DAMI, MEG-01. ${ }^{174}$ Moreover, overexpression of EDAG has been reported in a variety of human leukemia and lymphoma cells, ${ }^{175}$ suggesting that EDAG may play a role in hematopoietic transformation and leukemogenesis.

Enforced expression of EDAG in hematopoietic cell lines increased proliferation, enhanced survival, and inhibited differentiation. ${ }^{172}$ Overexpression of EDAG in NIH3T3 cells results in malignant transformation of cells, as characterized by cell morphology, anchorage-independent growth and tumorigenicity in nude mice. ${ }^{176}$ Overexpression of EDAG in HL-60 cells blocked monocyte and macrophage differentiation after pentahydroxytiglia myristate acetate induction. ${ }^{172}$ Overexpression of EDAG in pro-B Ba/F3 cells prolonged survival and increased the expression of $\mathrm{c}-\mathrm{Myc}, \mathrm{Bcl}-2$ and $\mathrm{Bcl}-\mathrm{xL}$ in the absence of IL-3. ${ }^{172}$ In contrast, downregulation of EDAG protein in K562 cells inhibited growth and colony formation, and enhanced sensitivity to erythroid differentiation induced by hemin. ${ }^{172}$ EDAG was shown to enhance the transcriptional activity of NF-kB (nuclear factor kappa B), and DNA-binding activity of NF-kB was sustained in $\mathrm{Ba} / \mathrm{F} 3$ cells overexpressing EDAG after IL-3 withdrawal. Inhibition of NF-kB activity resulted in promoting EDAG-induced Ba/F3 cell death. ${ }^{172}$ These results indicate that EDAG can block apoptosis, possibly through the activation of NF-kB. ${ }^{172}$ These results suggest that EDAG is an important regulator for proliferation and differentiation of hematopoietic cells, making its involvement in the HOXB4 phenotype very interesting.

An EDAG transgenic mouse was generated, using the human CD11a promoter to selectively express EDAG in hematopoietic tissues. ${ }^{173}$ EDAG overexpression in transgenic mice resulted in disrupted hematopoietic homeostasis. Myelopoiesis was enhanced with a progressive myeloid hyperplasia in the spleen and increased peripheral blood granulocytes in circulation. Lymphopoiesis was severely inhibited in the transgenic mice with $B$ lymphopoiesis blocked at the transition from pro/pre-B I to pre-B II stage in the bone marrow. Thymocyte development was also blocked at the most immature double negative stage. These data suggest that EDAG can act as a key regulator in primitive hematopoietic cells and its downregulation during maturation of blood cells may be necessary for maintaining homeostasis of the hematopoietic lineages. In the same report, EDAG was shown to be a transcriptional regulator 
which had transactivation activity and regulated the expression of several key transcription factors such as PU.1 and Pax5 in transgenic hematopoietic stem cells, ${ }^{173}$ suggesting that EDAG may positively or negatively regulate transcription of specific target genes. However, whether the effect of EDAG on these genes is direct needs further determination, since there is no evidence now that EDAG can bind DNA directly. 


\section{CHAPTER 2. IDENTIFICATION OF EARLY AND POTENTIAL DIRECT TARGET GENES OF HOXB4 USING AN INDUCIBLE HOXB4-ER ${ }^{\mathrm{T} 2}$ SYSTEM}

\subsection{Summary}

In order to understand the mechanism of HOXB4 mediated expansion of hematopoietic stem cells, we focused on identifying early and direct transcriptional targets of HOXB4 in primary murine hematopoietic progenitor cells. For this purpose, a HOXB4-ER ${ }^{T 2}$ fusion protein was created by molecular subcloning and introduced into undifferentiated bone marrow cells. Twelve hours after tamoxifen (TAM) exposure, HOXB4-ER ${ }^{\text {T2 }}$ transduced cells were functionally evaluated in myeloid progenitor expansion assays. Extracted RNA was analyzed by Affymetrix 430V2 chips, comparing expression patterns in HOXB4-ER ${ }^{\text {T2 }}$ versus GFP control vector-transduced cells. Four biologically independent experiments were performed to assess reproducibility. Stringent criteria were applied for mining microarray data using bioinformatics algorythms. Seventy seven probesets were identified that were differentially expressed $(>1.5$ fold, paired t-test $p<0.01$ ) in this early response to HOXB4 induction and were prioritized as candidate targets. Quantitative PCR was performed to validate the microarray data for specific genes of interest.

\subsection{Introduction}

Transcriptional regulation of gene expression is an essential function in all eukaryotes and is central to complex developmental control. Transcription factors are proteins that can regulate the expression of many physiologically important target genes including those that regulate proliferation and differentiation. The identification of transcription factors, transcription factor-target genes and transcriptional regulatory networks is therefore of fundamental importance in biology, because unraveling the logic of these gene regulation networks is necessary for a complete understanding how transcription factors contribute to biological phenotype. In my study, identifying molecules that promote hematopoietic stem cell expansion is a central goal, since significant expansion of hematopoietic stem cells would allow new transplant-based therapies. $3 ; 91$ However, in vitro expansion of hematopoietic stem cells has been difficult to achieve and often results in differentiation and apoptosis of developmentally primitive hematopoietic cells. Human HOXB4, a member of the homeobox transcription factor family, can promote hematopoietic stem and progenitor cell expansion both in vivo and in vitro when ectopically expressed in murine bone marrow cells. ${ }^{90 ; 95}$ HOXB4 overexpression also promotes specification of definitive hematopoietic stem cells from differentiating murine embryonic stem cells. ${ }^{146 ; 156}$ Since very little is known about the mechanisms of how HOXB4 promotes expansion of hematopoietic stem and progenitor cells, and because no specific 
direct target genes have yet been identified in hematopoietic cells, one objective of this study was to identify the downstream genetic pathways of HOXB4. In addition, direct target identification could also provide new factors for promoting hematopoietic stem and progenitor cell expansion.

There is only one published report identifying the targets of HOXB4 in hematopoietic cells. ${ }^{144}$ In this report, the downstream pathways of ectopic expressed HOXB4 were studied. Gene expression profiling assays were performed using enriched adult hematopoietic stem cells and embryonic derivatives that had been transduced with an inducible form of HOXB4. A set of overlapping gene expression change was generated from these two cellular systems and selected to represent common targets of HOXB4. ${ }^{144}$ These results showed that HOXB4 protected adult hematopoietic stem cells from the inhibitory effects mediated by the proinflammatory cytokine tumor necrosis factor alpha (TNF- $\alpha$ ), which likely inhibits the competitive repopulation advantage in the absence of HOXB4 overexpression. ${ }^{144}$ It was also demonstrated that HOXB4 activity and FGF signaling were related, since HOXB4-mediated expansion of adult and ES cell-derived hematopoietic stem cells was enhanced by inhibition of FGF receptors. ${ }^{144}$ In this report, however, no evidence was provided to show that any target genes from the generated candidate list was a direct transcriptional target of HOXB4. It was not clear if these pathway effects were primary versus secondary effects. Instead, their data suggest that multiple pathways downstream of HOXB4 could be regulated and they intertwined to modulate the self-renewal of hematopoietic stem cells promoted by HOXB4.

In my study, I utilized a traditional method for identifying the target genes of HOXB4 by an unbiased screen of its potential targets in a large scale gene expression profile analysis. Indeed, the activity of many nuclear localized proteins can be induced with hormones when these proteins are fused to hormone binding domains of steroid receptors such as estrogen receptor (ER). This allows pulsing of activity and enables study of acute effects. We established a HOXB4-ER ${ }^{T 2}$ inducible system to regulate the activity of HOXB4 in transduced hematopoietic cells by controlling the nuclear entry of HOXB4 with exogenous application of tamoxifen. When tamoxifen is absent, the HOXB4-ER ${ }^{\mathrm{T2}}$ fusion protein is sequestered by HSP90 in the cytosol. When tamoxifen is added, it diffuses into cells and binds to the ligand binding domain of ER ${ }^{\mathrm{T} 2}$ and thus releases the fusion protein from HSP90, which then can translocate into the nucleus and regulate the cis- elements of downstream genes for their activation or repression. Although the function of HOXB4 is most important in hematopoietic stem cell expansion, it also has a profound effect on hematopoietic progenitor cell expansion. It is relatively difficult to purify enough hematopoietic stem cells for microarray screening analysis, and there exists an established simple in vitro assay for evaluating HOXB4 function in hematopoietic myeloid progenitor cell expansion promptly. ${ }^{95}$ Therefore, I used this assay as a surrogate for hematopoietic stem cells in this study of HOXB4 downstream target identification. 


\subsection{Materials and Methods}

\subsubsection{Vector Construction}

Generation of MSCV-IRES-GFP vector was described previously. ${ }^{177}$ The human HOXB4 $\mathrm{cDNA}^{95}$ was kindly provided by Dr. Keith Humphries (Terry Fox Laboratory, Vancouver, Canada), and was cloned upstream of the IRES cassette into MSCV-IRES-GFP ${ }^{177 ; 178}$ backbone. For the MSCV-HOXB4-ER ${ }^{T^{2}}$-IRES-GFP vector, the mutated ligand binding domain of estrogen receptor $\left(E R^{\top 2}\right)$ was fused to the C-terminus of the HOXB4 CDNA with a linker with the following sequence: CLQNSDQRNE. The fusion HOXB4-ER ${ }^{T 2}$ gene was then cloned into the EcoRI and Xhol sites of MSCV-IRES-GFP vector.

\subsubsection{Retroviral Vector Preparation}

A polyclonal population of ecotropic retroviral producer GPE+86 cells ${ }^{179}$ was generated using a previously described approach. ${ }^{180 ; 181}$ Briefly, 2 ug pCAGGSVSVG and $4 \mu \mathrm{g}$ pEQ-PAM3(-E) helper plasmids were co-transfected with $4 \mu \mathrm{g}$ MSCV-cDNA-IRES-GFP vector into $8 \times 10^{6}$ cycling 293 T cells using FuGENE 6 reagent (Roche Applied Science, Indianapolis, IN) according to the manufacturer's instructions. Retroviral vector supernatants were harvested 48 hours post transfection, pooled, filtered through $0.45 \mu \mathrm{m}$ PVDF filter (Millipore, Billerica, MA), and used for transducing the cycling GPE+86 cells in the presence of $6 \mu \mathrm{g} / \mathrm{ml}$ polybrene. The transduced GFP positive GPE+86 cells were sorted and expanded in culture for establishing stable retroviral vector producer lines. The helper-free recombinant retroviral vector supernatants freshly collected from the GPE+86 producer cells were filtered through $0.45 \mu \mathrm{m}$ pore filter for transducing bone marrow cells.

\subsubsection{Separation, Transduction of Lin Bone Marrow Cells and Analysis}

Bone marrow cells were harvested from the tibias and femurs of 7 to 12 weeks old female C57BL/6J mice (The Jackson Laboratory, Bar Harbor, ME) as previously described, ${ }^{182}$ and were incubated with phycoerythrin (PE)-conjugated lineage antibody mixture (anti-mouse Mac-1, Gr-1, B220, CD4, CD8, Ter-119, Pharmingen, BD Biosciences, Franklin Lakes, NJ). Lin ${ }^{-}$bone marrow cells were enriched by depleting PE-labeled cells through anti-PE magnetic microbeads (Miltenyi Biotec, Auburn, CA) on autoMACS ${ }^{\text {TM }}$ separator (Miltenyi Biotec) according to the manufacturer's instructions. The Lin bone marrow cells were prestimulated for 2 days with $20 \mathrm{ng} / \mathrm{ml}$ recombinant mouse IL-3, $50 \mathrm{ng} / \mathrm{ml}$ human IL-6, and $50 \mathrm{ng} / \mathrm{ml}$ mouse SCF (R\&D Systems, Minneapolis, MN) in Dulbecco's modified Eagle medium (DMEM) supplemented with $15 \%$ fetal bovine serum (FBS), 100 units $/ \mathrm{ml}$ penicillin, $100 \mu \mathrm{g} / \mathrm{ml}$ streptomycin, $2 \mathrm{mM} \mathrm{L-glutamine} \mathrm{(R \& D}$ 
systems, Minneapolis, MN). The cells were then centrifuged at $2000 \mathrm{rpm}$ for 30 minutes at room temperature by using plates preloaded with $25 \mu \mathrm{g} / \mathrm{ml}$ RetroNectin (Takara, Madison, $\mathrm{WI}$ ) and retroviral vector supernatant generated as described above at an multiplicity of infection (MOI) of 10 to 20 . After 3 to 4 rounds of transduction in 2 consecutive days, cells were collected, washed with PBS, and then resuspended in the cytokine-supplemented medium described above. Proportions of the transduced GFP positive cells were determined by flow cytometry. Cells were cultured in the presence of TAM for additional time before plating into methylcellulose media for CFU-C assay, ${ }^{95}$ and RNA extraction for microarray.

\subsubsection{TAM Induction of HOXB4-ER ${ }^{T 2}$ and Myeloid Progenitor Assays}

$15.2 \mathrm{mg}$ of tamoxifen citrate (equivalent to $10 \mathrm{mg}$ of tamoxifen-TAM) tablet (Barr Laboratories, Pomona, NY) was dissolved in $20 \mathrm{ml} \mathrm{ddH}_{2} \mathrm{O}$ to obtain the stock $0.5 \mathrm{mg} / \mathrm{ml}$ TAM solution. The TAM solution was added directly into the bone marrow cells culture medium at the concentration $300 \mathrm{nM}$. TAM was withdrawn, and cells were plated for myeloid progenitor CFU-C assays established for HOXB4 expansion activity as previously described. ${ }^{95}$ In details, bone marrow cells were plated at 500 cells/ml in into MethoCult GF M3434 methylcellulose medium (StemCell Techologies, Vancouver, BC, Canada) containing various cytokines supporting growth of clonogenic myeloid progenitor cells. After 7 days, primary $\left(1^{\circ}\right)$ colonies were scored, collected, resuspended, and re-plated at $10^{5}$ cells $/ \mathrm{ml}$ density into the M3434 methylcellulose medium for 10 days culture. The generated secondary $\left(2^{\circ}\right)$ colonies were scored to quantitate the expansion of myeloid progenitors.

\subsubsection{Western Blotting}

Whole cell extracts were prepared from GPE +86 cells by M-PER Mammalian protein extraction reagent (Pierce, Thermo Scientific, Rockford, IL) supplemented with the protease inhibitors (Halt ${ }^{\mathrm{TM}}$ protease inhibitor cocktail kit, Thromo Scientific, Rockford, IL; Pefabloc SC Plus, Roche Applied Science, Indianapolis, IN). The proteins were then resolved on a $4-12 \%$ gradient NuPAGE Bis-Tris gel (Invitrogen, Carlsbad, California) and transferred to $0.45 \mu \mathrm{m}$ PVDF membrane. The primary monoclonal antibody (clone I12) (The Developmental Studies Hybridoma Bank, the University of lowa, lowa City, IA) was used for HOXB4 and HOXB4-ER ${ }^{\mathrm{T} 2}$ detection.

\subsubsection{Immunofluorescence Staining}

$1 \times 10^{5} \mathrm{Lin}^{-}$bone marrow cells were cytospined and fixed in $3.7 \%$ formaldehyde for 30 minutes at room temperature, washed and permeabilized in $1 \%$ Triton X- 
100 for 5 minutes at room temperature. Anti-HOXB4 antibody (clone I12) was added at $37^{\circ} \mathrm{C}$ for 1 hour, and after PBS washing, secondary antibody Alexa Fluor 594 goat anti-rat $\lg \mathrm{G}(\mathrm{H}+\mathrm{L})$ (Invitrogen, Carlsbad, California) was added to the cells for at room temperature for 1 hour. The nucleus was stained with TOTO-1 iodide (Invitrogen, Carlsbad, California). The stained slides were imaged with a Nikon TE2000-E inverted microscope equipped with a C1Si confocal system and an X40 Plan Apo objective.

\subsubsection{RNA Isolation, Affymetrix Array Hybridization and Analysis}

Total RNA was extracted from TAM-treated Lin ${ }^{-}$bone marrow cells using RNA STAT- $60^{\mathrm{TM}}$ reagent (Tel-Test, Friendswood, TX) according to the manufacturer's instructions. The RNA concentration and purity was determined by spectrophotometric analysis, integrity was confirmed by electrophoresis analysis using Agilent 2100 Bioanalyzer Lab-on-a-chip system (Agilent Technologies, Santa Clara, CA). Total RNA (100 ng) was processed in the Hartwell Center microarray core according to the Affymetrix eukaryote two-cycle target labeling protocol (http://www.affymetrix.com/support/technical/manual/expression manual.affx). Biotin-labeled cRNA $(20 \mu \mathrm{g})$ was hybridized overnight at $45^{\circ} \mathrm{C}$ to the Mouse430v2 GeneChip array. After staining and washing, arrays were scanned and expression values were summarized using the MAS5 algorithm as implemented in the GCOS v1.4 software (Affymetrix, Santa Clara, CA). Signals were normalized for each array by scaling to a $2 \%$ trimmed mean of 500 . Detection calls (Present, Absent and Marginal) were determined using the default parameters of the software. Signal values were log2-transformed prior to analysis. Differential expression between HOXB4-ER ${ }^{\top 2}$ transduced and GFP only-transduced cells was determined from four independent experiments using the paired t-test (S-Plus 6.2 software, TIBCO, Palo Alto, CA). Hierarchical clustering was performed using the Spotfire Decisionsite 9.0 software (TIBCO, Palo Alto, CA). Probeset annotations were obtained from the Affymetrix website (http://www.affymetrix.com/analysis/index.affx).

\subsubsection{Gene Expression Validation}

The RNA was first reversely transcribed with SuperScript ${ }^{\mathrm{TM}}$ III First-Strand Synthesis System for RT-PCR (Invitrogen, Carlsbad, California) by using oligodT primers. All gene-specific PCR primers were ordered from Quanti Tect Primer Assay kit (Qiagen, Valencia, CA) (Table 2-1).

The real-time PCR reactions were performed using QuantiTect ${ }^{\mathrm{TM}}$ SYBR Green PCR kit (Qiagen, Valencia, CA) following the manufacturer's instructions. The relative expression of each candidate gene was performed at least in triplicate

and calculated using the comparative quantitative comparative $\mathrm{C}_{\mathrm{T}}\left(2^{-\Delta \Delta C T}\right)$ 
Table 2-1. The ordered gene-specific PCR primers used in real-time PCR validation analysis.

\begin{tabular}{lll}
\hline $\begin{array}{l}\text { Gene } \\
\text { Symbol }\end{array}$ & QuantiTect Primers & Cat. No. \\
\hline Gapdh & Mm_Gapdh_3_SG QuantiTect Primer Assay & QT01658692 \\
Actb & Mm_Actb_2_SG Quanti Tect Primer Assay & QT01136772 \\
Thy1 & Mm_Thy1_1_SG QuantiTect Primer Assay & QT00245287 \\
Ephx2 & Mm_Ephx2_1_SG QuantiTect Primer Assay & QT00109165 \\
Slc22a3 & Mm_Slc22a3_1_SG QuantiTect Primer Assay & QT00131894 \\
Hemgn & Mm_Hemgn_1_SG QuantiTect Primer Assay & QT00106099 \\
Ms4a4c & Mm_Ms4a4c_1_SG QuantiTect Primer Assay & QT00175763 \\
PItp & Mm_PItp_2_SG_QuantiTect Primer Assay & QT01053311 \\
Mett11 & Mm_Mett1_1_SG QuantiTect Primer Assay & QT00153433 \\
Cldn11 & Mm_Cldn11_1_SG QuantiTect Primer Assay & QT00104195 \\
\hline
\end{tabular}


method with normalization to Gapdh internal control. The PCR primers of Actb were used for normalization in the real-time PCR reactions for detecting Hemgn expression in $\mathrm{Hoxb}^{-/-}$and wild type (WT) mice.

\subsection{Results}

\subsubsection{Retroviral Constructs and Expression of HOXB4-ER ${ }^{T 2}$ Fusion Protein}

To identify early and direct transcriptional targets of HOXB4 in murine bone marrow cells, a HOXB4-ER ${ }^{T 2}$ fusion gene was developed to allow temporally regulated HOXB4 activity by TAM. ${ }^{183}$ As controls, I used a retroviral vector expressing GFP protein as negative control, HOXB4 wild type gene as positive control under control of the MSCV promoter (Figure 2-1A). Expression of HOXB4 or HOXB4-ER ${ }^{\mathrm{T} 2}$ fusion protein were verified in established virus producer GPE+86 cells using western blotting analysis (Figure 2-1B).

\subsubsection{TAM Inducible Nuclear-Translocation of HOXB4-ER ${ }^{T 2}$}

To test whether HOXB4-ER ${ }^{\top 2}$ fusion protein can translocate into the nucleus after TAM treatment, TAM was added into the cultures of Lin $^{-}$bone marrow cells at a concentration $300 \mathrm{nM}$ for different time periods. TAM-induced nuclear translocation of HOXB4-ER ${ }^{\mathrm{T} 2}$ in transduced Lin bone marrow cells was confirmed by immunofluorescence staining 6 hours after TAM treatment (Figure 2-2). This experiment showed no nuclear localization of HOXB4 in the absence of TAM, implying that function would not be "leaky" in the absence of induction.

\subsubsection{TAM Inducible HOXB4-ER ${ }^{T 2}$ Activity Promoted Hematopoietic Progenitor Cell Expansion In Vitro}

An established expansion assay for myeloid progenitors was utilized for verifying TAM-dependent function. ${ }^{95}$ Lin $^{-}$bone marrow cells were purified and transduced with the HOXB4-ER ${ }^{\mathrm{T} 2}$ vector or the control GFP vector. The transduced cells were exposed to $300 \mathrm{nM}$ TAM in the culture medium for 12, 24, and 48 hours, washed, and then plated into semi-solid methylcellulose medium for myeloid progenitor colony forming unit cells (CFU-Cs) assay (Figure 2-3). TAM treatment for only 12 hours resulted in the generation of larger myeloid colonies, and more importantly, resulted in an increase in secondary colony number after re-plating (Figure 2-4A and B). Fluorescent microscope studies verified that the majority of secondary colonies were GFP positive and therefore contained and expressed the vector (Figure 2-5). These results prove that activation of HOXB4-ER ${ }^{\mathrm{T} 2}$ by TAM resulted in expansion of myeloid progenitor cells in vitro. This experiment also verified that no myeloid expansion occurred in 
A

MSCV-IRES-GFP

\begin{tabular}{|l|l|l|}
\hline MSCV LTR & IRES & GFP \\
\hline
\end{tabular}

MSCV-HOXBA-IRES-GFP

\begin{tabular}{|l|l|l|l|}
\hline MSCV LTR HOXB4 & IRES & GFP \\
\hline
\end{tabular}

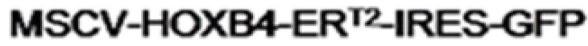

\begin{tabular}{|l|l|l|l|l|}
\hline MSCV LTR & HOXB4 & ER $^{\mathrm{T}}$ \\
\hline
\end{tabular}

HOXB4 Linker ER

\begin{tabular}{|l|l|l|}
\hline...$G P R A L Y$ & CLQNSDQRNE & MGASGD ... \\
\hline
\end{tabular}

$\mathbf{B}$

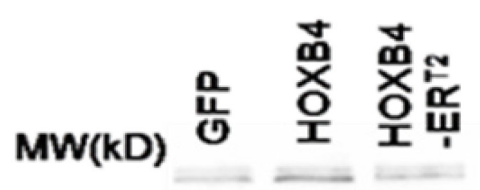

$78-$

$45.7-$

$32.5-$

Figure 2-1. Retroviral constructs and expression of HOXB4 and HOXB4$E R^{T 2}$ protein. (A) CDNA of HOXB4 and HOXB4-ER ${ }^{\top 2}$ was cloned upstream of the IRES cassette in MSCV-IRES-GFP backbone; The mutated ligand binding domain of estrogen receptor $\left(E R^{T 2}\right)$ was fused to the C-terminus of the HOXB4 CDNA with a linker CLQNSDQRNE. (B) Overexpression of HOXB4 or HOXB4$E R^{\top 2}$ was confirmed in retroviral vector producer GPE +86 cells by western blotting with a monoclonal anti-HOXB4 antibody (clone I12). 


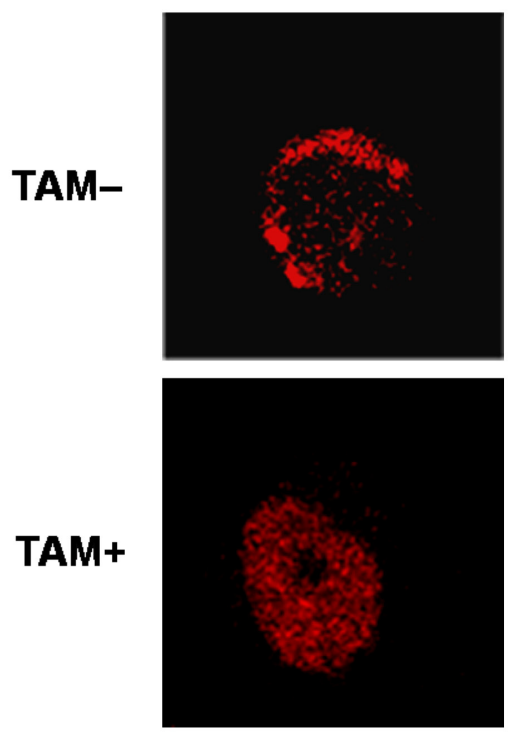

HOXB4-ERT2
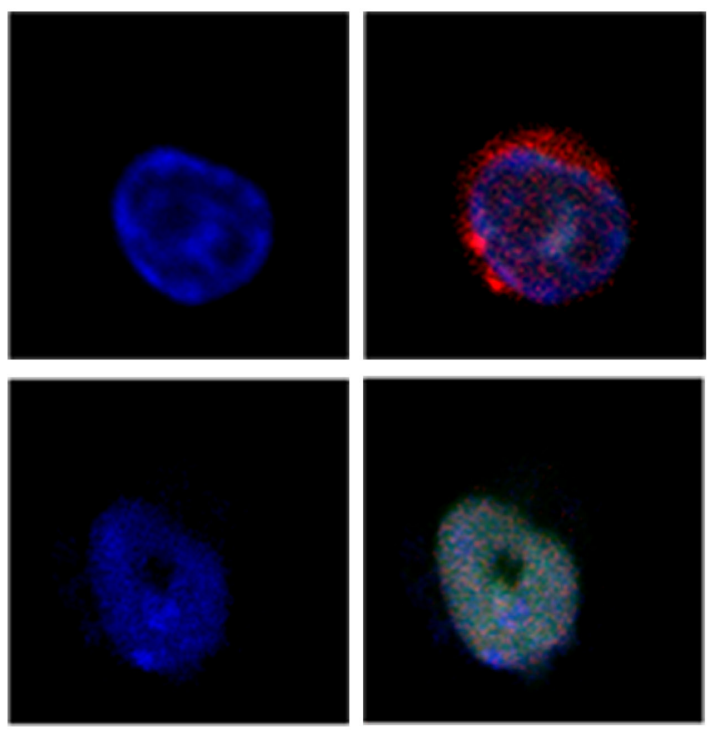

Nucleus

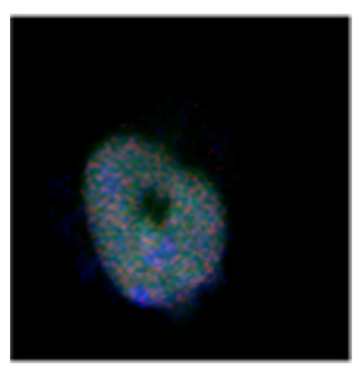

Merge

Figure 2-2. TAM-induced cytosol to nucleus translocation of HOXB4-ER ${ }^{\mathrm{T} 2}$ in Lin bone marrow cells confirmed by confocal microscopy. Red: HOXB4$\mathrm{ER}^{\mathrm{T} 2}$; Blue: nucleus dye. 

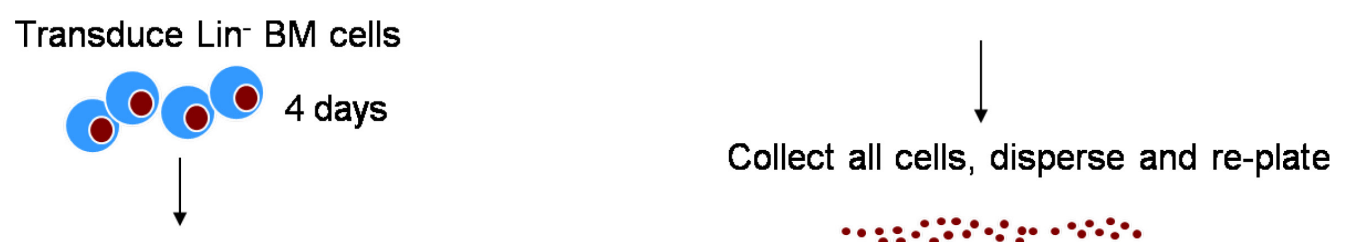

TAM for 12,24 , or $48 \mathrm{hr}$

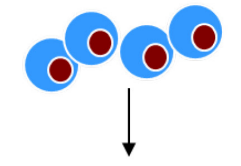

Wash out TAM

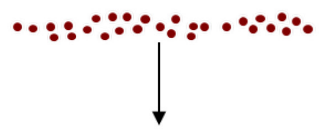

$1^{\circ}$ plating
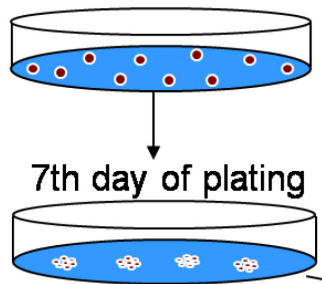

Collect all cells, disperse and re-plate
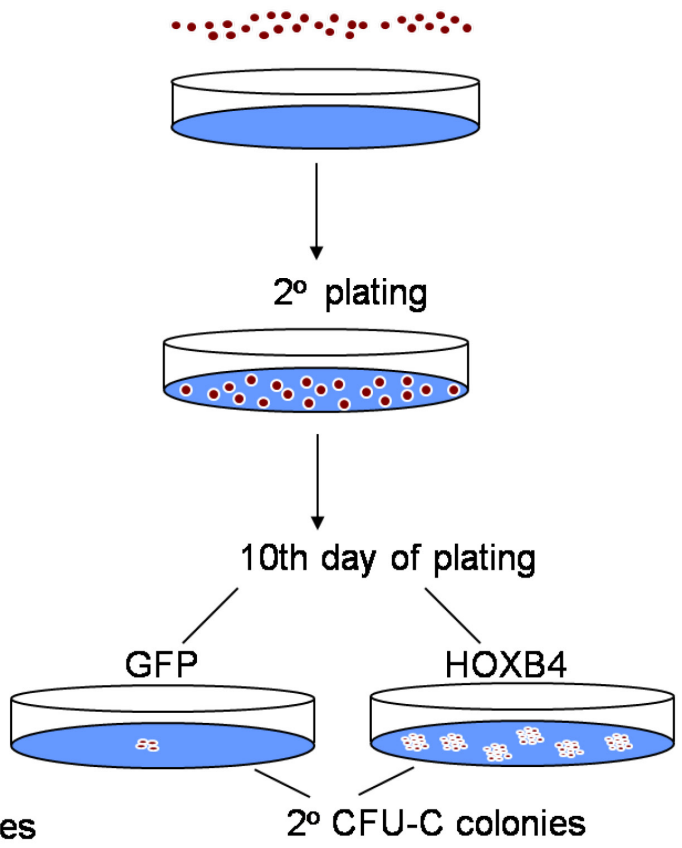

Figure 2-3. Schematic illustration of myeloid progenitor secondary CFU-C expansion assays. 
A

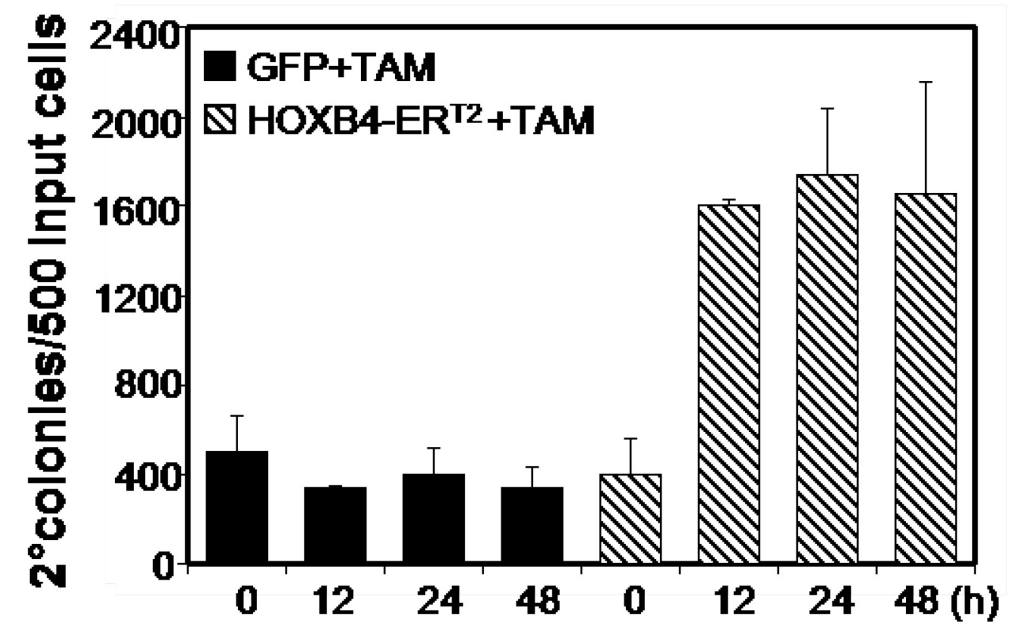

$\mathbf{B}$
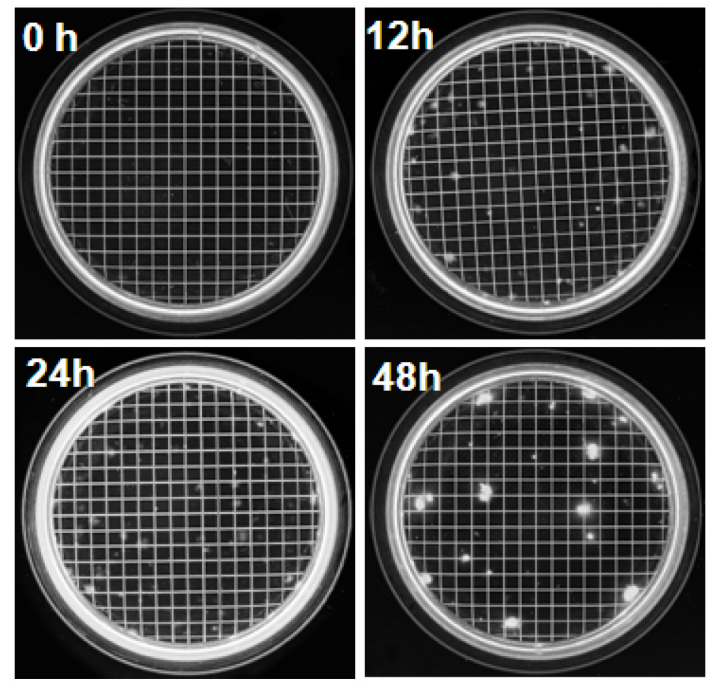

Figure 2-4. Effect of TAM-induced HOXB4-ER ${ }^{\mathrm{T} 2}$ activity in vitro. (A) TAM treatment for 12, 24 and 48 hours induced increase in secondary myeloid progenitor colony formation in HOXB4-ER ${ }^{\top 2}$-overexpressing Lin ${ }^{-}$bone marrow cells in vitro. (B) Pictures of the secondary myeloid progenitor colony plates from HOXB4-ER ${ }^{\text {T2 }}$-overexpressing cells treated with TAM for $0,12,24$ and 48 hours. 

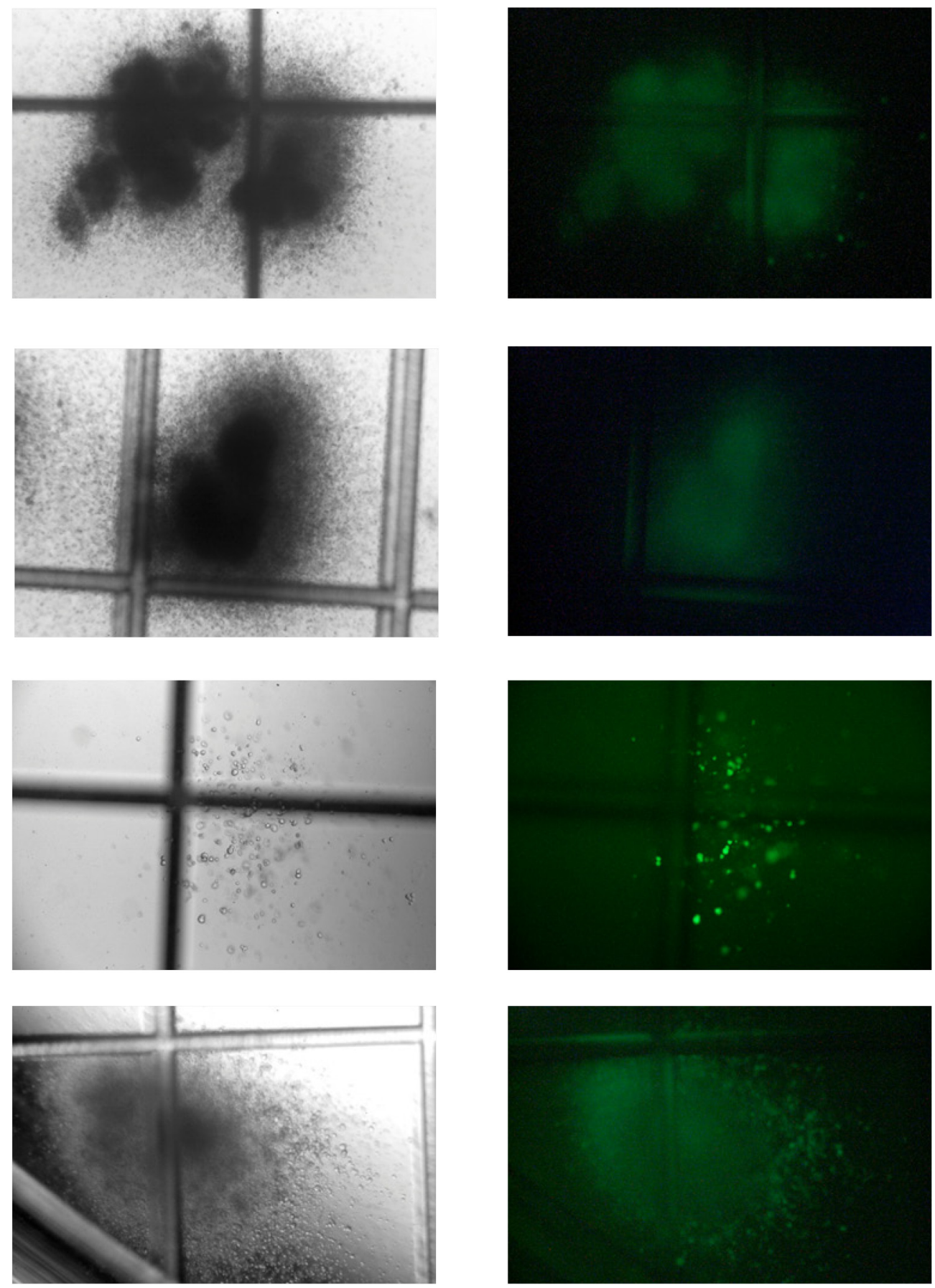

Figure 2-5. Secondary myeloid progenitor colonies from HOXB4-ER ${ }^{T^{2}}$ overexpressing Lin $^{-}$bone marrow cells were GFP positive under fluorescence microscopy. 
the absence of TAM, given that secondary CFU-C colony formation was low and equivalent to that seen with the GFP-only negative control vector.

\subsubsection{Identification of HOXB4 Early Downstream Targets by Expression Microarrays}

To identify early and direct downstream HOXB4 target genes involved in expansion of hematopoietic progenitor cells, RNA was extracted 12 hours after TAM-treatment of Lin ${ }^{-}$bone marrow cells transduced with either the HOXB4-ER ${ }^{\mathrm{T}}$ vector or with the GFP control vector, and was analyzed for differential gene expression using Affymetrix 430v2 chips (Figure 2-6). The transduction efficiency ranged from $60 \%$ to $70 \%$ in multiple experiments. Analysis of 4 independent transduction experiments showed good reproducibility in gene expression changes. The pearson correlation coefficient between all experimental samples ranged from 0.91 to 0.97 among probesets with more than one "Present" calls (Table 2-2), indicating that there was low experimental variation and that HOXB4 overexpression did not cause a change in global gene expression. First, we manually excluded those probesets showing more than 4 "Absent" calls in a total of 8 samples from the 4 experiments. A probeset was considered a potential downstream target of HOXB4 if the difference between the 2 mean expression values (HOXB4-ER ${ }^{\text {T2 }}$ vs. GFP) was at least 1.5 fold change, and the extent of the expression difference was statistically significant $(p<0.01)$ as evaluated by paired t-test method. We chose a 1.5 fold threshold due to the fact that $30 \sim 40 \%$ of the cells in a given sample were not transduced, hence the 1.5 fold change measured from the samples would underestimate what actually occurred in the transduced cells.

Seventy seven probesets were identified as being significantly changed by this analysis. Among them, 44 were upregulated (Table 2-3), and 33 were downregulated (Table 2-4) by HOXB4 induction. Hierarchical clustering analysis and heat map was generated for these 77 probesets from the 4 independent experiments (Figure 2-7 and Figure 2-8). Functional classification of those probesets by Gene Ontology annotations denoting different biological processes (Figure 2-9) showed a relatively high percentage of probesets involved in metabolism $(20,17 \%)$, signal pathways $(15,13 \%)$, and development and differentiation $(14,12 \%)$.

\subsubsection{Validation of the Selected HOXB4 Target Genes}

To validate the expression change seen in the microarray assays with HOXB4 induction, eight genes which showed a relatively large change in expression were selected for quantitative real time-PCR confirmation (Figure 2-10). 


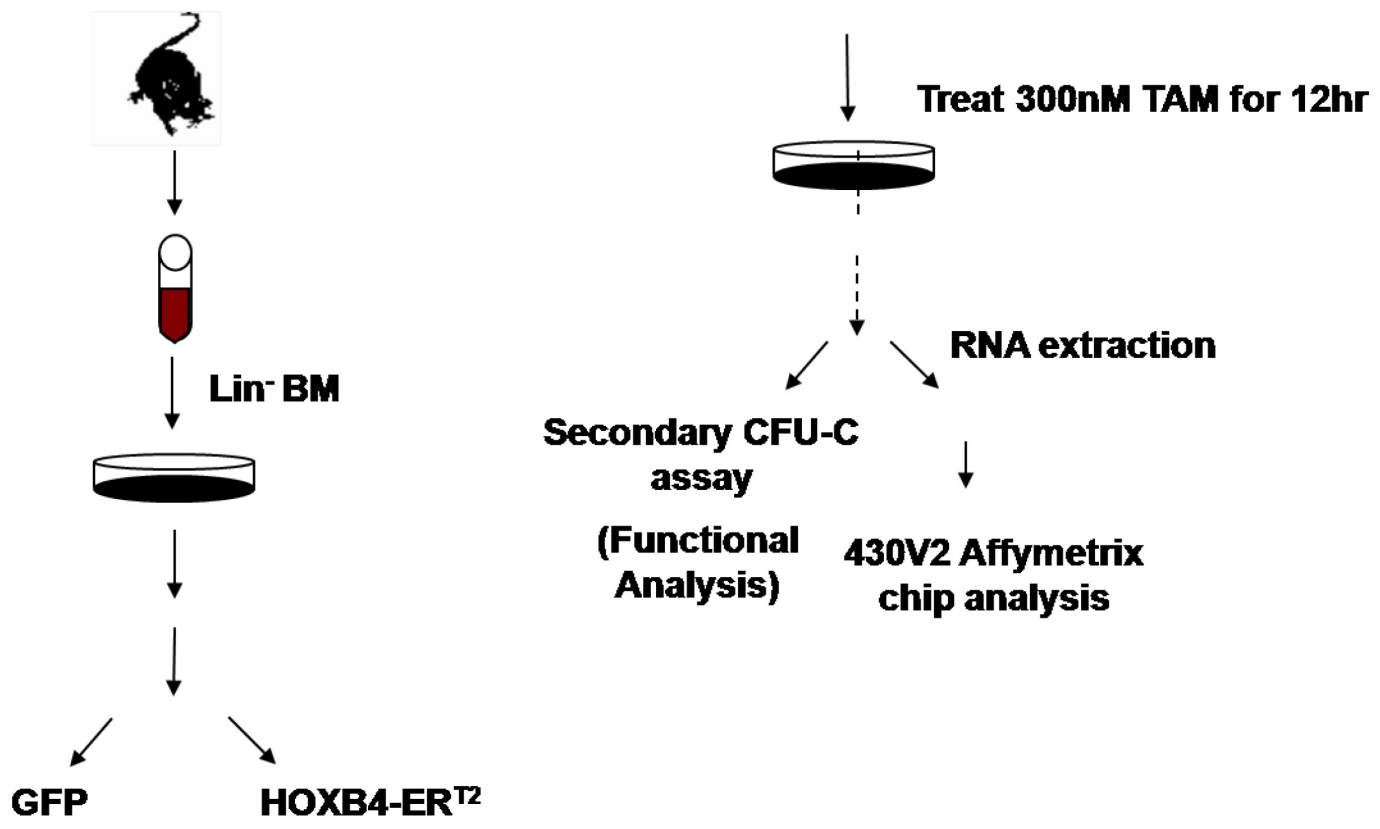

Figure 2-6. Schematic illustration of the inducible HOXB4-ER ${ }^{\top 2}$ system for target screening in Lin "bone marrow cells. 
Table 2-2. Pearson correlation coefficients between the 8 experimental samples with probesets $>1$ present call in microarray.

\begin{tabular}{|c|c|c|c|c|c|c|c|c|}
\hline Array & $\begin{array}{l}\text { Exp1 } \\
\text { GFP }\end{array}$ & $\begin{array}{c}\text { Exp1 } \\
\text { HOXB4- } \\
E^{T 2}\end{array}$ & $\begin{array}{c}\text { Exp2 } \\
\text { GFP }\end{array}$ & $\begin{array}{c}\text { Exp2 } \\
\text { HOXB4- } \\
E^{T 2}\end{array}$ & $\begin{array}{l}\text { Exp3 } \\
\text { GFP }\end{array}$ & $\begin{array}{c}\text { Exp3 } \\
\text { HOXB4- } \\
E^{T 2}\end{array}$ & $\begin{array}{c}\text { Exp4 } \\
\text { GFP }\end{array}$ & $\begin{array}{c}\text { Exp4 } \\
\text { HOXB4- } \\
E^{T 2}\end{array}$ \\
\hline $\begin{array}{c}\text { Exp1 } \\
\text { GFP } \\
\text { Exp1 }\end{array}$ & & 0.95 & 0.94 & 0.94 & 0.95 & 0.94 & 0.92 & 0.91 \\
\hline $\begin{array}{c}\text { HOXB4- } \\
E^{T 2}\end{array}$ & 0.95 & & 0.94 & 0.94 & 0.94 & 0.95 & 0.94 & 0.93 \\
\hline $\begin{array}{c}\text { Exp2 } \\
\text { GFP } \\
\text { Exp2 }\end{array}$ & 0.94 & 0.94 & & 0.94 & 0.94 & 0.94 & 0.93 & 0.92 \\
\hline $\begin{array}{c}\text { HOXB4- } \\
E R^{T 2}\end{array}$ & 0.94 & 0.94 & 0.94 & & 0.94 & 0.93 & 0.92 & 0.91 \\
\hline $\begin{array}{l}\text { Exp3 } \\
\text { GFP } \\
\text { Exp3 }\end{array}$ & 0.95 & 0.94 & 0.94 & 0.94 & & 0.95 & 0.94 & 0.93 \\
\hline $\begin{array}{c}\text { HOXB4- } \\
E R^{T 2}\end{array}$ & 0.94 & 0.95 & 0.94 & 0.93 & 0.95 & & 0.95 & 0.95 \\
\hline $\begin{array}{c}\text { Exp4 } \\
\text { GFP } \\
\text { Exp4 }\end{array}$ & 0.92 & 0.94 & 0.93 & 0.92 & 0.94 & 0.95 & & 0.95 \\
\hline $\begin{array}{c}\text { HOXB4- } \\
E^{T 2}\end{array}$ & 0.91 & 0.93 & 0.92 & 0.91 & 0.93 & 0.95 & 0.95 & \\
\hline
\end{tabular}


Table 2-3. The 44 probesets upregulated by HOXB4-ER ${ }^{\mathrm{T} 2}$.

\begin{tabular}{|c|c|c|c|c|}
\hline Probeset & $\begin{array}{l}\text { Gene } \\
\text { Symbol }\end{array}$ & $\begin{array}{l}\text { Fold } \\
\text { Chng }\end{array}$ & $\begin{array}{l}\text { Paired } t- \\
\text { Test } p \\
\text { Value }\end{array}$ & Gene Description \\
\hline$\overline{\text { 1421244_at }}$ & Esr1 & 14.42 & 5.56E-04 & estrogen receptor 1 (alpha) \\
\hline 1445285_at & $923010701 \mathrm{c}$ & 2.47 & 7.77E-03 & $\begin{array}{l}\text { Similar to histamine receptor } \mathrm{H} \\
3\end{array}$ \\
\hline 1423135_at & Thy1 & 2.34 & 8.99E-03 & thymus cell antigen 1 , theta \\
\hline 1448499_a_at & Ephx2 & 2.25 & 4.85E-03 & $\begin{array}{l}\text { epoxide hydrolase } 2 \text {, } \\
\text { cytoplasmic }\end{array}$ \\
\hline 1437260_at & Mmrn1 & 1.94 & $6.56 \mathrm{E}-03$ & multimerin 1 \\
\hline 1420444_at & Slc22a3 & 1.91 & $3.74 \mathrm{E}-03$ & $\begin{array}{l}\text { solute carrier family } 22 \text {, } \\
\text { member } 3\end{array}$ \\
\hline 1460130_at & EG4 & 1.90 & 4.95E-05 & predicted gene, EG433632 \\
\hline $141 \varepsilon$ & Her & & 8.05E-03 & hem \\
\hline 1459262_at & Mterfo & 1.80 & $9.14 \mathrm{E}-03$ & MTERF domain containing 1 \\
\hline 1425506_at & Mylk & 1.80 & 7.37E-03 & $\begin{array}{l}\text { myosin, light polypeptide } \\
\text { kinase }\end{array}$ \\
\hline 1429642_at & Anubl1 & 1.79 & 4.35E-03 & $\begin{array}{l}\text { AN1, ubiquitin-like, homolog } \\
\text { (Xenopus laevis) }\end{array}$ \\
\hline 1446745_at & $\mathrm{EG}$ & 1. & $5.59 \mathrm{E}-03$ & EG433637 \\
\hline 1419550_a_at & Stk & 1.78 & $8.12 \mathrm{E}-03$ & $\begin{array}{l}\text { serine/threonine kinase } 39 \text {, } \\
\text { STE20/SPS1 homolog (yeast) }\end{array}$ \\
\hline 143 & $\begin{array}{l}17000 \\
\text { Rik }\end{array}$ & 1 & -03 & $\begin{array}{l}\text { RIKEN cDNA 1700097N02 } \\
\text { gene }\end{array}$ \\
\hline 1446470_at & $\begin{array}{l}\text { ENSMUSG0 } \\
0000045520\end{array}$ & 1.77 & 6.65E-03 & $\begin{array}{l}\text { predicted gene, } \\
\text { ENSMUSG00000045520 }\end{array}$ \\
\hline 1441 & $\begin{array}{l}\text { A330024P } \\
\text { Rik }\end{array}$ & 76 & 5.30E-03 & $\begin{array}{l}\text { RIKEN cDNA A330024P14 } \\
\text { gene }\end{array}$ \\
\hline 1422317_a_at & $\| 1 \mathrm{rl} 1$ & 1. & 2.60E-03 & interleukin 1 receptor-like 1 \\
\hline 1421182_at & Clec1b & 1.73 & $1.38 \mathrm{E}-03$ & $\begin{array}{l}\text { C-type lectin domain family } 1 \text {, } \\
\text { member } b\end{array}$ \\
\hline 1435594_at & Arl6ip2 & 1.71 & $9.15 \mathrm{E}-03$ & $\begin{array}{l}\text { ADP-ribosylation factor-like } 6 \\
\text { interacting protein } 2\end{array}$ \\
\hline & $\mathrm{Fh}$ & & $9.35 \mathrm{E}-03$ & four and a half LIM domains 1 \\
\hline 1418892_at & Rhoj & 1.69 & $1.12 \mathrm{E}-04$ & $\begin{array}{l}\text { ras homolog gene family, } \\
\text { member J }\end{array}$ \\
\hline 1429691_at & $\begin{array}{l}5430405 N \\
\text { Rik }\end{array}$ & 1 & 1.07E-03 & $\begin{array}{l}\text { RIKEN cDNA 5430405N12 } \\
\text { gene }\end{array}$ \\
\hline
\end{tabular}


Table 2-3. Continued.

\begin{tabular}{|c|c|c|c|c|}
\hline Probeset & $\begin{array}{l}\text { Gene } \\
\text { Symbol }\end{array}$ & $\begin{array}{l}\text { Fold } \\
\text { Chng }\end{array}$ & $\begin{array}{l}\text { Paired t- } \\
\text { Test } p \\
\text { Value }\end{array}$ & Gene Description \\
\hline 1434869_at & Tdrd3 & 1.65 & $5.62 \mathrm{E}-03$ & tudor domain containing 3 \\
\hline 1458505_at & LOC552901 & 1.65 & 8.95E-03 & hypothetical LOC552901 \\
\hline 1429960_at & $\begin{array}{l}\text { 4930438A08 } \\
\text { Rik }\end{array}$ & 1.63 & $6.62 \mathrm{E}-03$ & $\begin{array}{l}\text { RIKEN cDNA 4930438A08 } \\
\text { gene }\end{array}$ \\
\hline 1418186_at & Gstt1 & 1.62 & 5.36E-03 & $\begin{array}{l}\text { glutathione S-transferase, } \\
\text { theta } 1\end{array}$ \\
\hline 1451038_at & Apln & 1.61 & $2.22 \mathrm{E}-03$ & apelin \\
\hline 1443633_at & $\begin{array}{l}1700086006 \\
\text { Rik }\end{array}$ & 1.60 & 7.26E-03 & $\begin{array}{l}\text { RIKEN cDNA } 1700086006 \\
\text { gene }\end{array}$ \\
\hline 1438575_a_at & 3974589 & 1.60 & $6.23 \mathrm{E}-03$ & Transcribed locus \\
\hline 1444869_at & $\mathrm{H} 3145 \mathrm{H} 06$ & 1.60 & 7.05E-03 & Transcribed locus \\
\hline 1422927_at & Yipf7 & 1.60 & $4.81 \mathrm{E}-03$ & Yip1 domain family, member 7 \\
\hline 1442884_at & $\mathrm{Hgf}$ & 1.58 & $5.55 \mathrm{E}-03$ & hepatocyte growth factor \\
\hline 1435710_at & Al661384 & 1.58 & $1.86 \mathrm{E}-04$ & expressed sequence Al661384 \\
\hline 1430522_a_at & Vamp5 & 1.57 & 4.61E-03 & $\begin{array}{l}\text { vesicle-associated membrane } \\
\text { protein } 5\end{array}$ \\
\hline 1440227_at & Slc5a3 & 1.57 & 9.71E-03 & $\begin{array}{l}\text { solute carrier family } 5 \text { (inositol } \\
\text { transporters), member } 3\end{array}$ \\
\hline 1439755_at & Sipa1l1 & 1.55 & $3.44 \mathrm{E}-03$ & $\begin{array}{l}\text { Signal-induced proliferation- } \\
\text { associated } 1 \text { like } 1\end{array}$ \\
\hline 1438664_at & Prkar2b & 1.54 & $2.84 \mathrm{E}-03$ & $\begin{array}{l}\text { protein kinase, cAMP } \\
\text { dependent regulatory, type II } \\
\text { beta }\end{array}$ \\
\hline 1444433_at & 30077393 & 1.53 & $9.38 \mathrm{E}-04$ & Transcribed locus \\
\hline 1420688_a_at & Sgce & 1.52 & $1.24 \mathrm{E}-03$ & sarcoglycan, epsilon \\
\hline 1457110_at & Pank1 & 1.52 & $6.25 \mathrm{E}-03$ & pantothenate kinase 1 \\
\hline 1450235_at & Fgd3 & 1.52 & $1.33 \mathrm{E}-03$ & $\begin{array}{l}\text { FYVE, RhoGEF and PH } \\
\text { domain containing } 3\end{array}$ \\
\hline 1432362_at & Cenpp & 1.52 & $1.66 \mathrm{E}-03$ & centromere protein $\mathrm{P}$ \\
\hline 1431874_at & $\begin{array}{l}\text { 4931429L15 } \\
\text { Rik }\end{array}$ & 1.51 & 4.89E-03 & $\begin{array}{l}\text { RIKEN cDNA 4931429L15 } \\
\text { gene }\end{array}$ \\
\hline 1446719_at & Atad2b & 1.51 & 7.03E-03 & $\begin{array}{l}\text { ATPase family, AAA domain } \\
\text { containing } 2 B\end{array}$ \\
\hline
\end{tabular}


Table 2-4. The 33 probesets downregulated by HOXB4-ER ${ }^{\mathrm{T} 2}$.

\begin{tabular}{|c|c|c|c|c|}
\hline Probeset & $\begin{array}{l}\text { Gene } \\
\text { Symbol }\end{array}$ & $\begin{array}{l}\text { Fold } \\
\text { Chng }\end{array}$ & $\begin{array}{l}\text { Paired t- } \\
\text { Test } p \\
\text { Value }\end{array}$ & Gene Description \\
\hline 1416003_at & Cldn11 & -3.85 & $4.11 \mathrm{E}-03$ & claudin 11 \\
\hline 1447683_x_at & Mett11 & -2.44 & $2.39 \mathrm{E}-03$ & methyltransferase-like 1 \\
\hline 1435529_at & $\begin{array}{l}\text { OTTMUSG0 } \\
0000016644\end{array}$ & -2.33 & 8.63E-03 & $\begin{array}{l}\text { predicted gene, } \\
\text { OTTMUSG00000016644 }\end{array}$ \\
\hline 1417963_at & Pltp & -2.27 & $1.92 \mathrm{E}-03$ & phospholipid transfer protein \\
\hline 1435792_at & Csprs & -2.27 & $1.90 \mathrm{E}-03$ & component of Sp100-rs \\
\hline 1420671_x_at & Ms4a4c & -2.22 & $1.55 \mathrm{E}-03$ & $\begin{array}{l}\text { membrane-spanning 4- } \\
\text { domains, subfamily A, member } \\
\text { 4C }\end{array}$ \\
\hline 1450616_at & Ear5 & -2.04 & 4.27E-03 & $\begin{array}{l}\text { eosinophil-associated, } \\
\text { ribonuclease A family, member } \\
5\end{array}$ \\
\hline 1443956_at & Zfp397 & -2.00 & $5.84 \mathrm{E}-03$ & zinc finger protein 397 \\
\hline 1416023_at & Fabp3 & -2.00 & 7.63E-03 & $\begin{array}{l}\text { fatty acid binding protein } 3 \text {, } \\
\text { muscle and heart }\end{array}$ \\
\hline 1429984_at & $\begin{array}{l}5730455013 \\
\text { Rik }\end{array}$ & -1.96 & 8.95E-03 & $\begin{array}{l}\text { RIKEN cDNA } 5730455013 \\
\text { gene }\end{array}$ \\
\hline 1417500_a_at & Tgm2 & -1.85 & $6.51 \mathrm{E}-03$ & $\begin{array}{l}\text { transglutaminase } 2, \mathrm{C} \\
\text { polypeptide }\end{array}$ \\
\hline 1423963_at & $\begin{array}{l}\text { LOC1000456 } \\
29\end{array}$ & $3-1.79$ & $1.60 \mathrm{E}-03$ & $\begin{array}{l}\text { similar to myocardial ischemic } \\
\text { preconditioning upregulated } \\
\text { protein } 2\end{array}$ \\
\hline 1452361_at & Rnf20 & -1.75 & 2.32E-04 & ring finger protein 20 \\
\hline 1430388_a_at & Sulf2 & -1.72 & $2.84 \mathrm{E}-03$ & sulfatase 2 \\
\hline 1437347_at & Ednrb & -1.72 & 9.05E-04 & endothelin receptor type B \\
\hline 1416289_at & Plod1 & -1.69 & 4.00E-04 & $\begin{array}{l}\text { procollagen-lysine, } 2 \text { - } \\
\text { oxoglutarate } 5 \text {-dioxygenase } 1\end{array}$ \\
\hline 1449195_s_at & Cxcl16 & -1.67 & $3.40 \mathrm{E}-03$ & $\begin{array}{l}\text { chemokine (C-X-C motif) ligand } \\
16\end{array}$ \\
\hline
\end{tabular}


Table 2-4. Continued.

\begin{tabular}{|c|c|c|c|c|}
\hline Probeset & $\begin{array}{l}\text { Gene } \\
\text { Symbol }\end{array}$ & $\begin{array}{l}\text { Fold } \\
\text { Chng }\end{array}$ & $\begin{array}{l}\text { Paired } t- \\
\text { Test } p \\
\text { Value }\end{array}$ & Gene Description \\
\hline 1421604_a_at & KIf3 & -1.64 & $8.60 \mathrm{E}-04$ & Kruppel-like factor 3 (basic) \\
\hline 1428535_at & $\begin{array}{l}\text { 9430020K01 } \\
\text { Rik }\end{array}$ & -1.64 & $6.12 \mathrm{E}-03$ & $\begin{array}{l}\text { RIKEN cDNA } 9430020 K 01 \\
\text { gene }\end{array}$ \\
\hline 1460648_at & $\mathrm{Nr} 2 \mathrm{f6}$ & -1.64 & $8.25 \mathrm{E}-03$ & $\begin{array}{l}\text { nuclear receptor subfamily } 2 \text {, } \\
\text { group F, member } 6\end{array}$ \\
\hline 1424986_s_at & Fbxw7 & -1.61 & $2.52 \mathrm{E}-03$ & $\begin{array}{l}\text { F-box and WD-40 domain } \\
\text { protein } 7, \text { archipelago homolog } \\
\text { (Drosophila) }\end{array}$ \\
\hline 1425658_at & Cd109 & -1.56 & $3.26 \mathrm{E}-03$ & CD109 antigen \\
\hline 1450488_at & Ccl24 & -1.56 & 8.04E-03 & $\begin{array}{l}\text { chemokine (C-C motif) ligand } \\
24\end{array}$ \\
\hline 1417865_at & Tnfaip1 & -1.54 & 5.89E-03 & $\begin{array}{l}\text { tumor necrosis factor, alpha- } \\
\text { induced protein } 1 \text { (endothelial) }\end{array}$ \\
\hline 1424498_at & $\begin{array}{l}\text { 5730596K20 } \\
\text { Rik }\end{array}$ & -1.54 & 8.06E-03 & $\begin{array}{l}\text { RIKEN cDNA 5730596K20 } \\
\text { gene }\end{array}$ \\
\hline 1454676_s_at & Ticam1 & -1.54 & $9.53 \mathrm{E}-03$ & $\begin{array}{l}\text { toll-like receptor adaptor } \\
\text { molecule } 1\end{array}$ \\
\hline 1446528_at & Scyl2 & -1.52 & 8.18E-03 & SCY1-like 2 (S. cerevisiae) \\
\hline 1452014_a_at & $\operatorname{lgf} 1$ & -1.52 & 8.07E-03 & insulin-like growth factor 1 \\
\hline 1422054_a_at & Skil & -1.52 & $9.93 \mathrm{E}-03$ & SKI-like \\
\hline 1416654_at & Slc31a2 & -1.52 & 3.57E-03 & $\begin{array}{l}\text { solute carrier family } 31 \text {, } \\
\text { member } 2\end{array}$ \\
\hline 1426847_at & Sirt4 & -1.52 & 1.38E-03 & $\begin{array}{l}\text { sirtuin } 4 \text { (silent mating type } \\
\text { information regulation } 2 \\
\text { homolog) } 4 \text { (S. cerevisiae) }\end{array}$ \\
\hline 1439348_at & $\mathrm{S} 100 \mathrm{a} 10$ & -1.52 & 7.14E-03 & $\begin{array}{l}\text { S100 calcium binding protein } \\
\text { A10 (calpactin) }\end{array}$ \\
\hline 1416044_at & Flii & -1.52 & $1.40 \mathrm{E}-03$ & $\begin{array}{l}\text { flightless I homolog } \\
\text { (Drosophila) }\end{array}$ \\
\hline
\end{tabular}




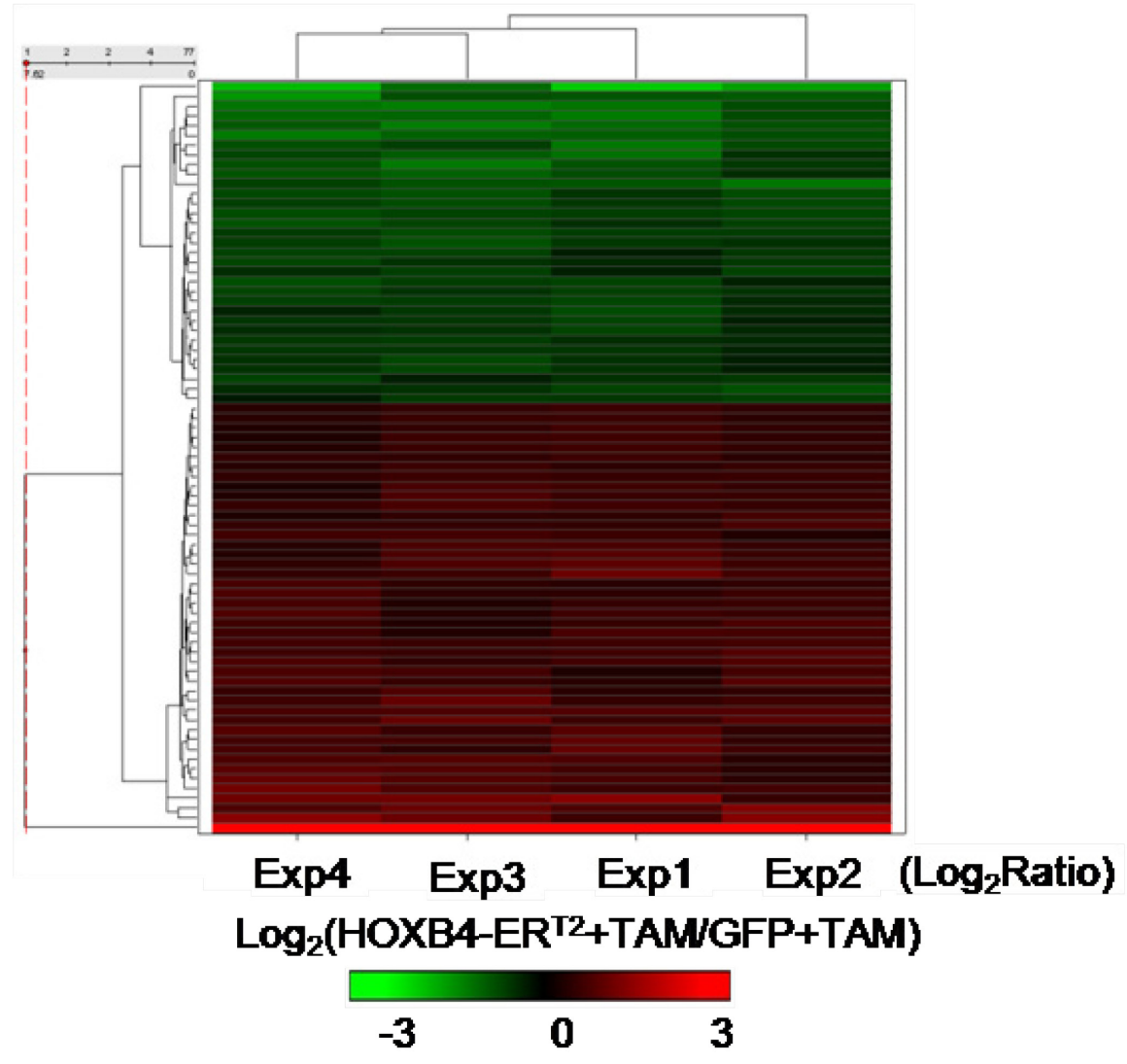

Figure 2-7. Hierarchical cluster diagram and heat map of the 77 candidate probesets selected from 4 biologically-independent experiments using the $\log _{2}$-ratio of TAM-induced HOXB4-ER ${ }^{\text {T2 }}$ vs. TAM-induced GFP expression values observed within each of the 4 experiments. 


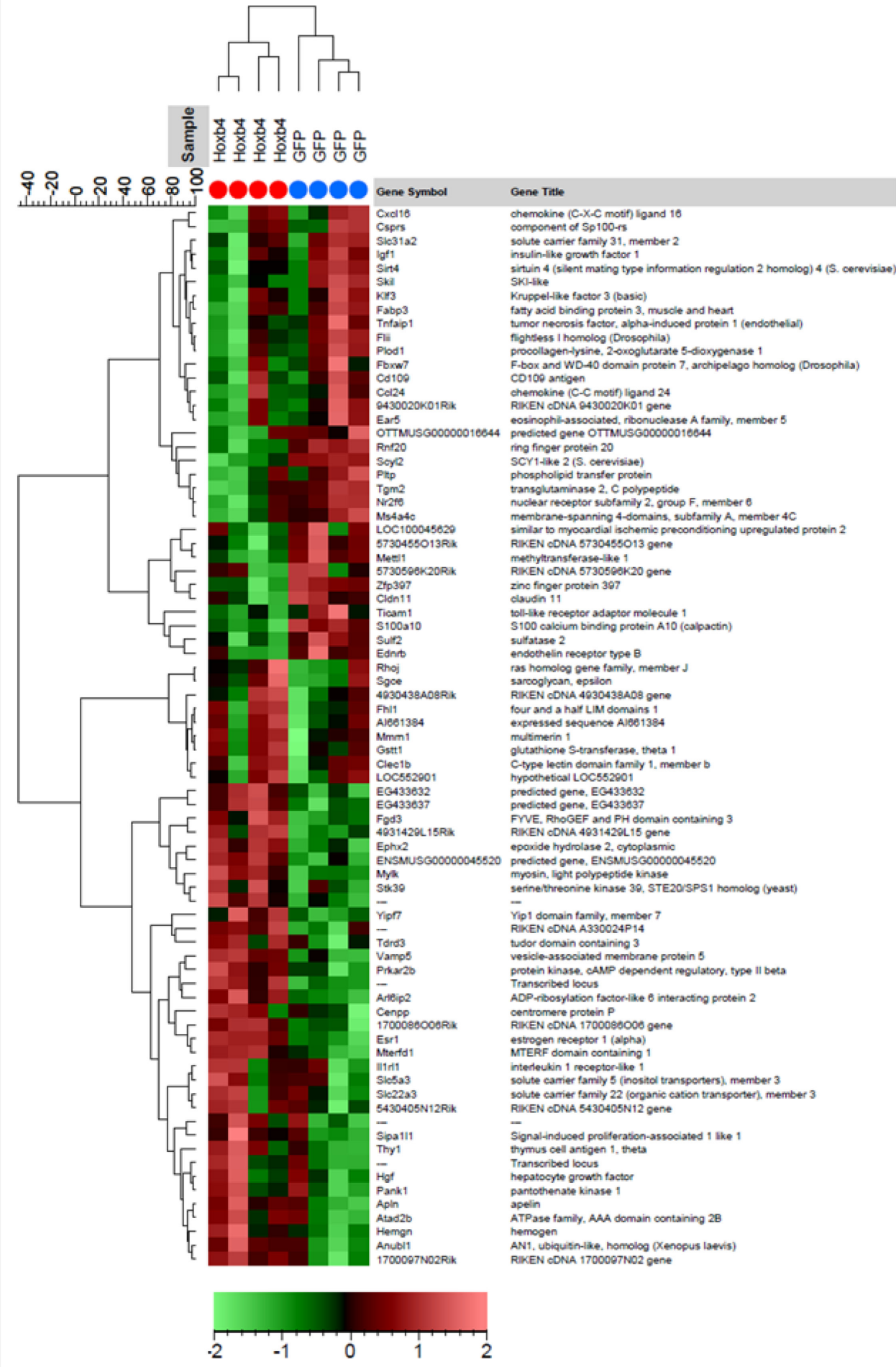

Figure 2-8. Hierarchical cluster diagram and heat map of the 77 candidate probesets selected from 4 biologically-independent experiments using the normalized z-score. 


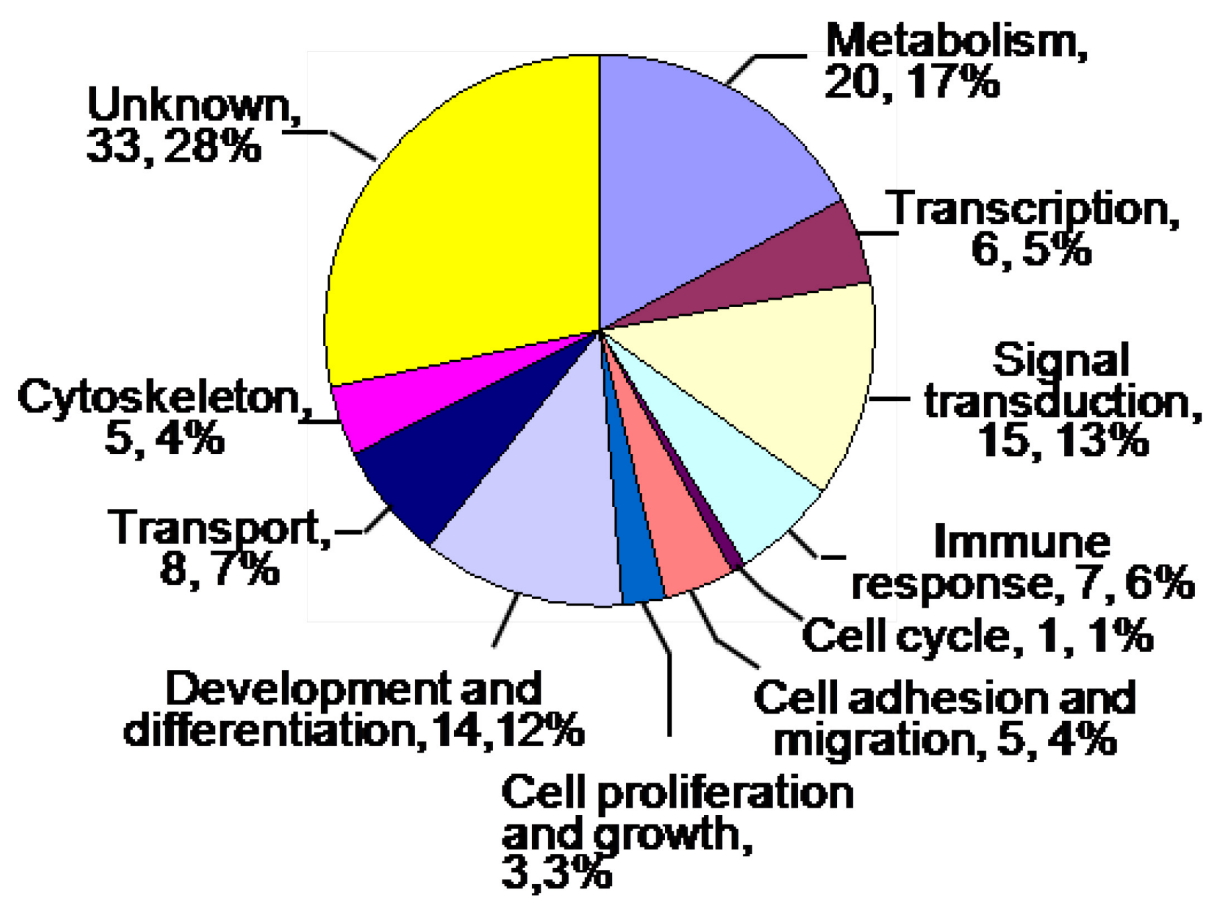

Figure 2-9. Functional classification of the 77 probesets by using gene ontology annotations. Some probesets were classified into more than one category, thus the sum of all 11 groups is more than 77 . 


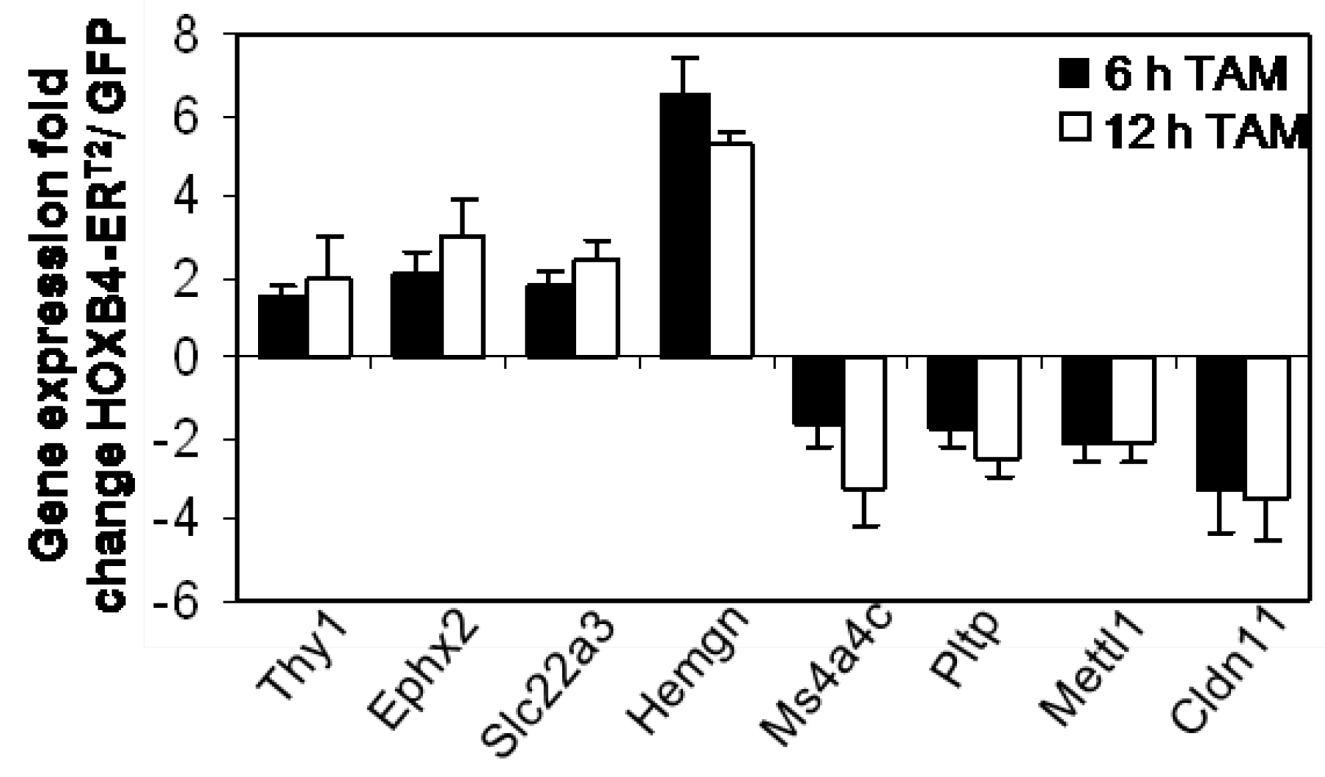

Figure 2-10. Quantitative real-time PCR confirmation of the expression change of the selected 8 candidate target genes from independent experiments with TAM treatment for 6 hours or 12 hours in HOXB4-ER ${ }^{\text {T2 }}$ vector-transduced and GFP control vector-transduced Lin ${ }^{-}$bone marrow cells. 
$\Delta \Delta \mathrm{C}_{\mathrm{T}}$ method was used for quantification of relative gene expression levels. The housekeeping gene Gapdh was used as an internal control for gene expression normalization. The plot of template cDNA dilution vs. $\Delta \mathrm{C}_{\mathrm{T}}$ was close to zero, verifying the approximately equal PCR amplification effiencies of the tested genes and the endogenous Gapdh control. Then the $\mathrm{C}_{\mathrm{T}}$ values of the tested target gene and Gapdh control in HOXB4-ER ${ }^{T 2}$ sample were compared to the $\mathrm{C}_{\mathrm{T}}$ values of them in the GFP sample to generate a relative expression change of this specific target gene between the 2 samples.

The quantitative PCR results correlated fairly well with the microarray data and also showed that changes in the gene expression present in 12 hours TAMtreated samples were also present at 6 hours after TAM treatment, consistent with an immediate transcriptional effect.

\subsubsection{Hemgn mRNA Expression Level in Bone Marrow and c-kit ${ }^{+}$Scal-1 ${ }^{+}$Lin $^{-}$ Cells from Hoxb4 ${ }^{-1-}$ Mice}

Hemgn was one most interesting gene that was upregulated at a relatively high level compared to other genes and appeared to be an interesting gene for further study. Hemgn is a nuclear protein specifically expressed in hematopoietic stem and progenitor cells that appears to protect cells from apoptosis and differentiation. ${ }^{170 ; 172}$ We obtained whole bone marrow and c-kit ${ }^{+}$Scal- $1^{+}$Lin $^{-}$bone marrow cells from Hoxb4 $4^{--}$mice and showed by real-time PCR that Hemgn mRNA was significantly decreased relative to that seen in control cells from wild type mice (Figure 2-11), indicating that Hemgn may also be regulated by endogenous Hoxb4. The $\Delta \Delta \mathrm{C}_{\mathrm{T}}$ method was used for quantification of the relative gene expression level.

\subsection{Discussion}

The function of transcription factors may be confounded by factors such as redundancy, pleiotropic overlap, sequential downstream pathway activation due to the ectopic expression. The use of inducible expression cassettes or inducible nuclear targeting of transcription factors may overcome these limitations, and such systems have been widely used for direct target identification of transcription factors. In our study, an inducible HOXB4-ER ${ }^{\mathrm{T} 2}$ system was generated and proved to work well in vitro, recapitulating the functional phenotype caused by HOXB4 overexpression. Most importantly, the inducible construct did not cause any detectable leakiness in the absence of TAM treatment. Our use of the inducible HOXB4-ER ${ }^{\mathrm{T} 2}$ system allowed the identification of very early and potential direct downstream effectors of HOXB4, with the controlled application of TAM. A significant advantage of this system is that it ensures the detected gene expression changes are based on biological coherence instead of random events, since the myeloid progenitor CFU-C 


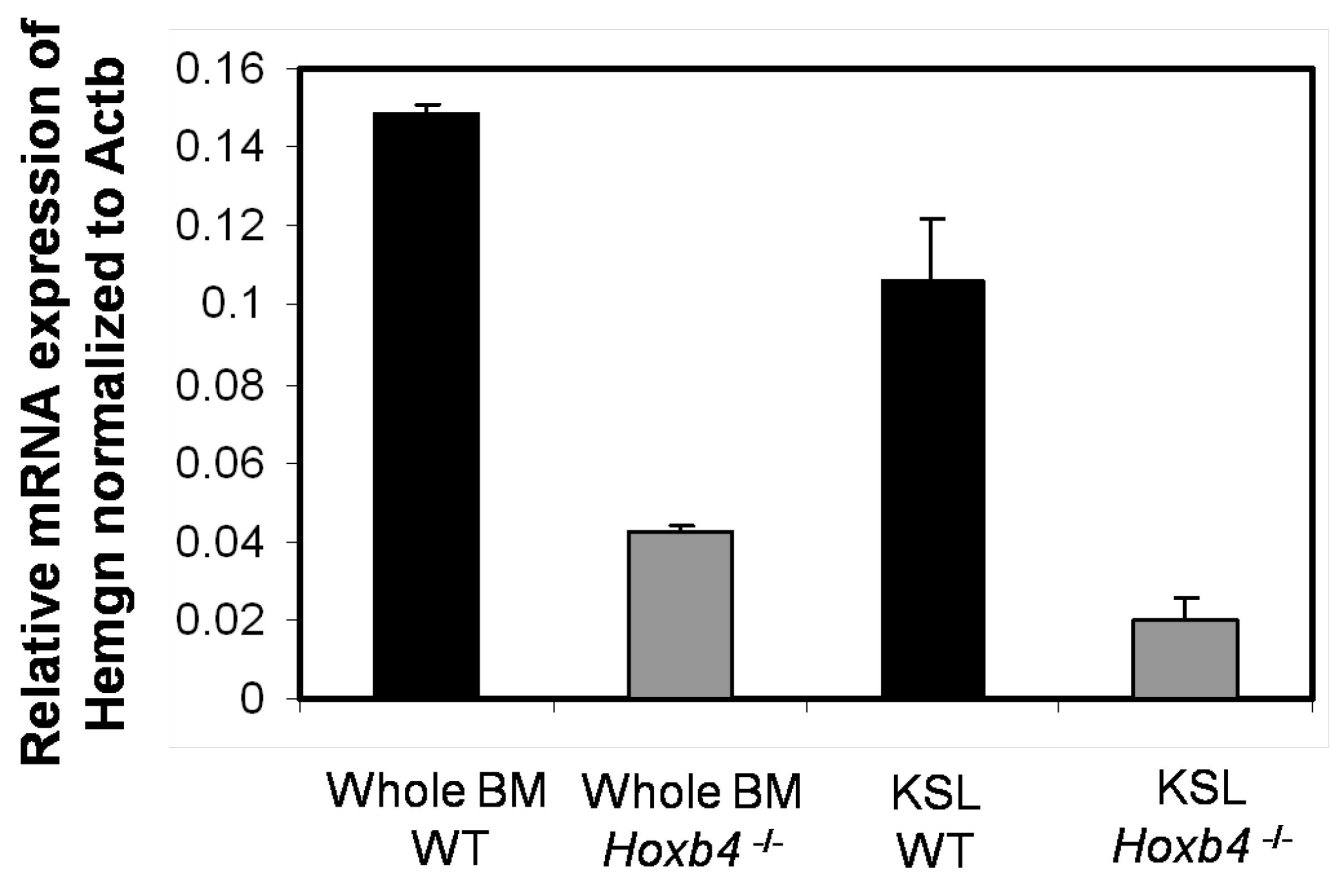

Figure 2-11. Quantitative real-time PCR detection of relative expression level of Hemgn mRNA in whole bone marrow or c-kit ${ }^{+}$Scal-1 ${ }^{+} \mathrm{Lin}^{-}(\mathrm{KSL})$ cells from $\mathrm{Hoxb}^{-\%}$ mice compared to wild type (WT) mice. The calculation is based on the quantitative comparative $\mathrm{C}_{\mathrm{T}}\left(2^{-\Delta \Delta \mathrm{CT}}\right)$ method with normalization to Actb internal control. Four biological independent experiments were applied in this assay. 
assays were validated in advance to reflect the function of induced HOXB4 in promoting hematopoietic progenitor cell expansion.

The gene profiling assay for the expression change between the HOXB4-ER ${ }^{T 2}$ plus TAM samples versus the GFP plus TAM samples is a reasonable comparison method to exclude the effect caused by TAM itself. The criteria for screening the targets of HOXB4 were stringent in our study. A 1.5 fold threshold for gene differential change was selected since only 60 to $70 \%$ of the cells in a given sample were transduced, hence the 1.5 fold change measured from the samples would underestimate what actually occurred in the transduced cells. I tried once to sort transduced bone marrow cells for the gene screening assay, but due to the variance generated from the sorting process to the cells, I decided to use non-sorted cells in this study. We found HOXB4 activation did not induce a global gene expression change, as the gene expression showed high reproducibility between and within each experiment, indicated by the fact that the pearson correlation coefficients between the 8 experimental samples ranged from 0.91 to 0.97 . This data indicate that the chromatin structure was not altered to initiate a global change in gene expression, and only a small subset of specific genes were impacted by HOXB4 activation. Hierarchical clustering analysis and heat map generated from the identified 77 probesets from the 4 independent experiments further validated the high reproducibility between experiments. In this small target gene list, we found that 44 genes were reproducibly upregulated and 33 of them were downregulated by HOXB4, indicating that HOXB4 can both activate and repress downstream gene expression, which is consistent with previous results. ${ }^{140}$ The identified target genes were shown to be involved in a variety of cellular processes, of which, the ones classified in signaling pathways, development and differentiation, cell proliferation and growth might be the most significant targets for further focus.

Further PCR confirmation of the selected genes with highest fold change between HOXB4-ER ${ }^{\mathrm{T} 2}$ and GFP samples validated the quality of the data generated from microarray screening. As 12 hours TAM treatment was used in microarray assays, to discern whether the detected gene expression change also exists at an earlier time point, samples from 6 hours of TAM treatment were also included and showed that most of the studied genes were also altered at 6 hours.

A gene called Hemgn was pursued further based on its relative high degree of expression change. After reviewing the literature, we found that relatively little is known about Hemgn, which was cloned in $2001 .^{170}$ Its expression is restricted to testis and hematopoietic tissues. In this regard, its spatial and temporal expression pattern is similar to endogenous Hoxb4. Overexpression of human HEMGN in transgenic mice caused disruption of the balance of myelopoiesis and lymphopoiesis, ${ }^{173}$ suggesting an important role in hematopoiesis. This promoted us to study Hemgn further as an important HOXB4 target gene. 
We recognize that other potential HOXB4 target genes identified from microarray screening are also likely to contribute to the downstream signaling network of HOXB4 in conveying its function. This 77 candidate list can serve as a resource for further research on HOXB4-promoted expansion effect. We did select several genes with relative high degrees of changes in expression between HOXB4-ER ${ }^{\top 2}$ and GFP samples based on microarray and real-time PCR results. I cloned their cDNA upstream of the IRES cassette in the MSCVIRES-GFP retroviral vector for overexpression study. Stable GPE+86 vector producer cell lines overexpressing those individual target genes were generated to provide vector supernatant for bone marrow transduction. Bone marrow cells overexpressing individual target genes were tested for myeloid progenitor expansion ability in the secondary CFU-C assay as a prompt in vitro evaluation method for their function. In this assay, however, only Hemgn showed ability to promote myeloid progenitor expansion in vitro. Two of the upregulated genes by HOXB4: Thy1 or Ephx2, when overexpressed, did not cause expansion of myeloid progenitor cells. In contrast, one of the downregulated genes by HOXB4: Ms4a4c, inhibited expansion of myeloid progenitor cells when it was overexpressed. This suggests that downregulation of Ms4a4c could be a mechanism of HOXB4's expansion ability. In general, the data provided us an additional practical reason to focus on investigating Hemgn instead of other candidates in our study.

Interestingly, the mRNA expression of Hemgn also showed correlation with endogenous Hoxb4 expression. A 5 -fold decrease in Hemgn mRNA was found in Hoxb4-deficient c-kit ${ }^{+}$Scal- $1^{+} \mathrm{Lin}^{-}$bone marrow cells compared to wild type cells. This data further strengthens the hypothesis that Hemgn is regulated by HOXB4. Because the expression of Hemgn can still be detected in the absence of Hoxb4, the residual amount of Hemgn expression possibly still effectively maintains the normal hematopoietic stem and progenitor cell expansion. Since Hox4 paralog members show redundancy in their functions, ${ }^{167}$ investigation of whether Hemgn could be further downregulated in Hoxa4;b4;c4;d4 quadruplex knockout mice would be interesting. 


\section{CHAPTER 3. DEMONSTRATION THAT HEMGN IS A DIRECT TRANSCRIPTIONAL TARGET OF HOXB4}

\subsection{Summary}

Among the 77 candidate target genes identified by microarray screening using the HOXB4-ER ${ }^{\top 2}$ inducible system, Hemogen (Hemgn), a newly discovered nuclear protein specifically expressed in hematopoietic cells with reported functions in blocking apoptosis, inhibiting differentiation, and promoting survival, is a particularly interesting one for us to pursue further. Here we show that Hemgn is indeed the first identified direct transcriptional target of HOXB4 in primary bone marrow cells. Promoter activity of Hemgn was increased by HOXB4 in MEL cells in transient co-activation assays, implying direct transactivation of Hemgn by HOXB4. Furthermore, we show HOXB4 directly bound to the promoter fragment of Hemgn in electrophoresis mobility shift assay (EMSA). Most importantly, we validated that HOXB4 was recruited in vivo to the Hemgn promoter region in primary 5-FU treated hematopoietic progenitor cellenriched bone marrow cells, through chromatin immunoprecipitation (ChIP) assay.

\subsection{Introduction}

Traditionally, experimental approaches have sought to identify target genes of transcription factors by measuring gene expression in loss- or gain-of-function assays, while transcription factor-binding to transcriptional regulatory regions has been evaluated using EMSA, co-transfection or ChIP assays. Classic microarray for gene expression profiling or genome-wide location data for transcription factor binding from ChIP-chip experiments does not provide definitive evidence of target regulation. These screening methods should always be combined with functional biological experiments for targets confirmation.

Among the potential candidates from the microarray screening, we decided to focus on Hemgn, based on its relatively high degree of upregulated expression induced by HOXB4, its known hematopoietic expression pattern, and given the fact that relatively little is known about this gene and its potential function in hematopoiesis. Hemgn is a recently discovered nuclear protein that is restrictively expressed in testis and hematopoietic tissues. ${ }^{170 ; 171}$ It shows a spatial and temporal expression pattern in hematopoietic sites during embryogenesis, and is expressed in the blood islands of yolk sac, then in fetal liver, and finally in the adult spleen and bone marrow. ${ }^{170}$ In adult bone marrow, Hemgn is predominantly expressed in primitive progenitor and stem cell populations, but not in terminally differentiated mature blood cells. ${ }^{170}$ Human HEMGN, also called EDAG, shows a similar expression pattern in bone marrow cells and interestingly, maps to chromosome 9q22 which contains well known 
leukemic breakpoints. ${ }^{170}$ Relatively high levels of EDAG expression have been observed in human acute myeloid leukemia bone marrow cells. ${ }^{175}$ In transgenic mice in which EDAG was driven by human CD11a promoter, enhancement of myelopoiesis and suppression of lymphopoiesis was found, suggesting its regulatory role in hematopoiesis. ${ }^{1 / 3}$ To our knowledge, Hemgn has not been knocked out in a mouse genetic model so that the null phenotype is unknown.

The activity of the Hemgn promoter containing 2975 bp of 5 ' flanking sequence and $196 \mathrm{bp}$ of $5^{\prime}$ UTR was tested in a luciferase assay and was reported to be active in a hematopoietic cell line K562, but not in nonhematopoietic cell lines. ${ }^{184}$ This promoter region was also able to drive the transcription of a lac $Z$ transgene in hematopoietic tissues in transgenic mice. ${ }^{184}$ GATA1 was demonstrated to bind to 2 GATA binding sites in this promoter region of Hemgn in the K562 cells and to transactivate it. EMSA showed direct binding of GATA1 to the Hemgn promoter in MEL cells. ChIP assay showed the recruitment of GATA1 to Hemgn promoter in Meg-01 AML cell line. This study suggests that the Hemgn promoter contains critical regulatory elements for its transcription in hematopoietic tissues and Hemgn is a direct target of GATA1 in leukemia cell line K562. ${ }^{184}$ Except for the data from this report, there is no other literature on the transcriptional regulation of Hemgn and no information exists for its regulation in primary hematopoietic cells.

In this study, after using the traditional method for identifying the target genes in a gain-of-function model of HOXB4, we then focused on validating a specific target, Hemgn, with supplemental co-transfection, EMSA, and ChIP assays.

\subsection{Materials and Methods}

\subsubsection{Transient Cotransfection Luciferase Assay}

In the promoter region of Hemgn, $-1887 \mathrm{bp} \sim+213 \mathrm{bp}$ region containing the 4 putative HOXB4 binding sites based on sequence analysis. The transcription start site (TSS) was cloned upstream of a luciferase reporter gene in pGL4.12Luc2CP vector (Promega, Madison, WI). $1 \mu \mathrm{g}$ pGL4.12-Luc2CP vector and 0.5 ng renilla control $p G L 4.75-R / u c / C M V$ vector were transiently transfected with either $1 \mu \mathrm{g}$ MSCV-IRES-GFP control or $1 \mu \mathrm{g}$ MSCV-HOXB4-IRES-GFP vector into $2 \times 10^{5} \mathrm{MEL}$ cells with $4 \mu \mathrm{l}$ Fugene 6 reagent (Roche Applied Science, Indianapolis, IN). Luciferase activity was measured by dual-luciferase reporter assay system (Promega, Madison, WI) 48 hours after transfection and was normalized to renilla activity. 


\subsubsection{Electrophoretic Mobility Shift Assay (EMSA)}

A GST-tag was fused to the N-terminus of HOXB4 protein and the GSTHOXB4 fusion protein was expressed and purified through pET-GST Fusion System 42 (Novagen, EMD Chemicals Inc, San Diego, CA). Synthetic single strand DNA oligos were biotin-labeled with Biotin 3' END DNA labeling kit (Pierce, Holmdel, NJ) and annealed. The reactions were processed using LightShift Chemiluminescent EMSA Kit (Pierce, Holmdel, NJ) according to the manufacturer's instructions. Briefly, $20 \mathrm{fmol}$ biotin-labeled probes were incubated with $5 \mu \mathrm{g}$ GST or GST-HOXB4 protein, together with $2 \mu \mathrm{l} 10 \mathrm{x}$ binding buffer, $1 \mu \mathrm{g}$ poly(dl.dC), HBC loading buffer (20\% Glycerol, 20 mM HEPES (PH7.8), $0.2 \mathrm{mM}$ EDTA, $1 \mathrm{mM}$ DTT) and ultrapure water to a final $20 \mu \mathrm{l}$ reaction volumn. For the cold probe competition reactions, 4 pmol unlabelled target oligos were additionally added in the reactions. For supershift analysis, $1 \mu \mathrm{g}$ Anti-HOXB4 antibody (clone 112) (The Developmental Studies Hybridoma Bank, the University of lowa, lowa City, IA) was incubated with $5 \mu \mathrm{g}$ GST or GST-HOXB4 for 30 minutes on ice preceding the DNA binding reaction. The DNA-protein complexes were separated by $6 \%$ TBE DNA retardation gel (Invitrogen, Carlsbad, California) and transferred to Biodyne B Nylon Membrane (Pierce, Rockford, IL). The probe containing complex in the crosslinked membrane was recognized by streptavidinHRP and incubated with the substrate. The luminescent signal was captured by $\mathrm{X}$-ray film. The sequence of the WT probe for the promoter of Hemgn is: 5' ACACTCTGCTAATTACAGCCTTT 3' (-1562bp -1540bp); and sequence of the mutant probe is: 5' ACACTCTGCAGCATACAGCCTTT 3'.

\subsubsection{Separation and Transduction of 5-FU Treated Bone Marrow Cells}

Bone marrow cells were harvested 4 days after intraperitoneal injection of mice with $150 \mathrm{mg} / \mathrm{kg} 5-\mathrm{FU}^{185 ; 186}$ from the tibias and femurs of 7 to 12 weeks old female C57BL/6J mice (The Jackson Laboratory, Bar Harbor, ME) as previously described. ${ }^{182}$ Red blood cells were lysed with BD pharm Lyse ${ }^{T M}$ lysing buffer (BD Biosciences, San Jose, CA). The 5-FU treated bone marrow cells were prestimulated for 2 days with the cytokine-supplemented DMEM medium described in Chapter 2. The method for transduction of the cells with retroviral vector supernatant collected from the GPE +86 producer cells were described in Chapter 2. After transduction, cells were collected for ChIP assay.

\subsubsection{Chromatin Immunoprecipitation (ChIP) Assay}

ChIP assay was performed using ChIP-IT TM Express kit (Active Motif, Carlsbad, CA) following the manufacturer's instructions. Briefly, the transduced 5 -FU treated bone marrow cells were fixed with $1 \%$ formaldehyde for 10 minutes at room temperature, and the chromatin from $2 \times 10^{7}$ cells was sonicated into fragments $(\sim 500 \mathrm{bp})$. The chromatin-protein complex derived from $2.5 \times 10^{6}$ cells 
was immunoprecipitated with either $1 \mu \mathrm{g}$ anti-HOXB4 antibody (I12) or $1 \mu \mathrm{g}$ IgG2A isotype control antibody (R\&D Systems, Minneapolis, MN). The semiquantitative PCR (Tag PCR core kit, Qiagen, Valencia, CA) was set as follow: $94^{\circ} \mathrm{C} 5$ minutes; 35 cycles alternating between $94^{\circ} \mathrm{C}$ for 1 minute, $60^{\circ} \mathrm{C}$ for 1 minute and $72^{\circ} \mathrm{C}$ for 1 minute; $72^{\circ} \mathrm{C} 10$ minutes. The quantitative real-time PCR was performed with SYBR Green DNA incorporation method by using QuantiTect ${ }^{\mathrm{TM}}$ SBBR Green PCR kit (Qiagen) and was carried out on StepOnePlus ${ }^{\mathrm{TM}}$ Real-Time PCR System (Applied Biosystems, Foster City, CA), and was analyzed with quantitative comparative $C_{T}\left(2^{-\Delta \Lambda C T}\right)$ method normalized to input control samples using StepOne Software v2.0 (Applied Biosystem, Foster City, CA). The PCR reaction program was set up as follow: $95^{\circ} \mathrm{C} 10 \mathrm{~min} ; 40$ cycles alternating between $95^{\circ} \mathrm{C} 15$ seconds and $60^{\circ} \mathrm{C} 1$ minute. The melting curves were generated by the program to validate one single PCR product for each reaction. The results were analyzed in quantitative comparative $C_{T}\left(2^{-\Delta \Delta C T}\right)$ method normalized to input control samples using StepOne Software v2.0 (Applied Biosystem, Foster City, CA).

The PCR primers are listed below:

1. Semi-quantitative and quantitative real time PCR primers for amplifying $-54 \mathrm{bp}$ $\sim+89 \mathrm{bp}$ in the promoter of Actb:

Forward: 5' CGCGAGCACAGCTTCTTTG 3'

Reverse: 5' GGTGTCCGTTCTGAGTGATCC 3'

2. Semi-quantitative PCR primers for amplifying regions in Hemgn promoter:

(1). -1568bp -1447bp region:

Forward: 5' GCCACCACACTCTGCTAATTAC 3'

Reverse: 5' CGGAGAGGAAACTAGGAAAG 3'

(Also used in quantitative real time PCR)

(2). $-543 b p \sim-228 b p$ region:

Forward: 5' GGTGAGCAGTGGAGATTAGC 3'

Reverse: 5' AGGGAGATCCCAGGGTTTAG 3'

3. Quantitative real-time PCR primers for amplifying regions in Hemgn promoter:

(1). $-1731 b p \sim-1542 b p$ region

Forward: 5' AGGATGGTGAGTTGAGGTCGGC 3'

Reverse: 5' AGGCTGTAATTAGCAGAGTGTGGTGG 3'

(2). - -1485bp -1336bp region

Forward: 5' ACATTTCAAATGCTAGCCCCTTTCC 3'

Reverse: 5' CACCTTCTCTCTCTTTATGCACACATGT 3' 
(3). $-436 b p \sim-332 b p$ region

Forward: 5' GTGAAAACATAGCCACACCTAGGAAGAT 3'

Reverse: 5' CCACTCTTGCCTGTTTCATACACTTCTT 3'

\subsection{Results}

\subsubsection{The Promoter of Hemgn Contains Potential HOXB4 Binding Motifs}

Inspection of the promoter region (-1887bp +213bp) of Hemgn revealed 4 potential HOXB4 binding sites with a "TAAT" core and preferred flanking nucleotides (Figure 3-1). We know that the "TAAT" core sequence is relatively common and could appear in any potential cis-regulatory elements, which could be in the promoter region 5' to Hemgn, or in an intron, or in the 3' untranslated region of Hemgn. Here we only examined the $\sim 2 \mathrm{~kb}$ promoter region of Hemgn, and all the following functional evaluation were based on this region. The existence of multiple "TAAT" sites in this $\sim 2 \mathrm{~kb}$ fragment suggests a potential increased abundance of HOXB4 on this promoter region due to the widespread binding sites. The specificity of potential HOXB4 binding is possible based on preferred flanking nucleotides of the 4 "TAAT".

\subsubsection{The Promoter of Hemgn Was Transcriptionally Activated by HOXB4}

The $-1887 \mathrm{bp} \sim+213 \mathrm{bp}$ region containing the 4 potential HOXB4 sites and the transcription start site (TSS) was cloned upstream of a luciferase reporter gene and this construct was tested in a transient transfection assay in mouse erythroleukemia (MEL) cells. When this construct was co-transfected with an internal renilla control vector and an MSCV vector expressing either HOXB4 or a GFP control protein, HOXB4 significantly increased the luciferase activity in MEL cells (Figure 3-2), indicating that the promoter of Hemgn was directly activated by HOXB4. However, the limitation of this assay is that first, the Hemgn promoter fragment used in the transfected luciferase reporter plasmid may not mimic the true transcriptional regulation of the endogenous gene. Second, both plasmid DNA and HOXB4 protein expression are introduced transiently and at high episomal copy numbers; far beyond the physiological level, potentially forcing non-physiologic the DNA-protein interactions. Third, a cell line instead of primary cells in this assay based on transfection efficiency considerations. Therefore, we sought independent confirmation of this putative interaction.

\subsubsection{HOXB4 Directly Bound to the Promoter Fragment of Hemgn In Vitro}

Among the 4 putative HOXB4 binding sites (Figure 3-1), site 1 (-1555bp $1548 \mathrm{bp}$ ) contains the most favored flanking nucleotides for high affinity HOXB4 


\begin{tabular}{|c|c|c|c|c|}
\hline Site 1 & Site 2 & Site 3 & Site 4 & \\
\hline $\begin{array}{ll}5^{\prime} & -1555 \sim \\
& -1548 b p\end{array}$ & $\begin{array}{l}-1519 \sim \\
-1512 \mathrm{pp}\end{array}$ & $\begin{array}{l}-1424 \sim \\
-1417 b p\end{array}$ & $\begin{array}{l}-385 \\
-378 b p\end{array}$ & \\
\hline _ GCTAATTA & _CTAATTA & CAATTACA & AGTAATCA & TSS \\
\hline CGATTAAT & AGATTAAT & GTTAATGT & TCATTAGT & \\
\hline
\end{tabular}

Figure 3-1. Schematic illustration of the multiple putative HOXB4 binding sites on the promoter region of Hemgn (site 1, 2, 3 and 4).

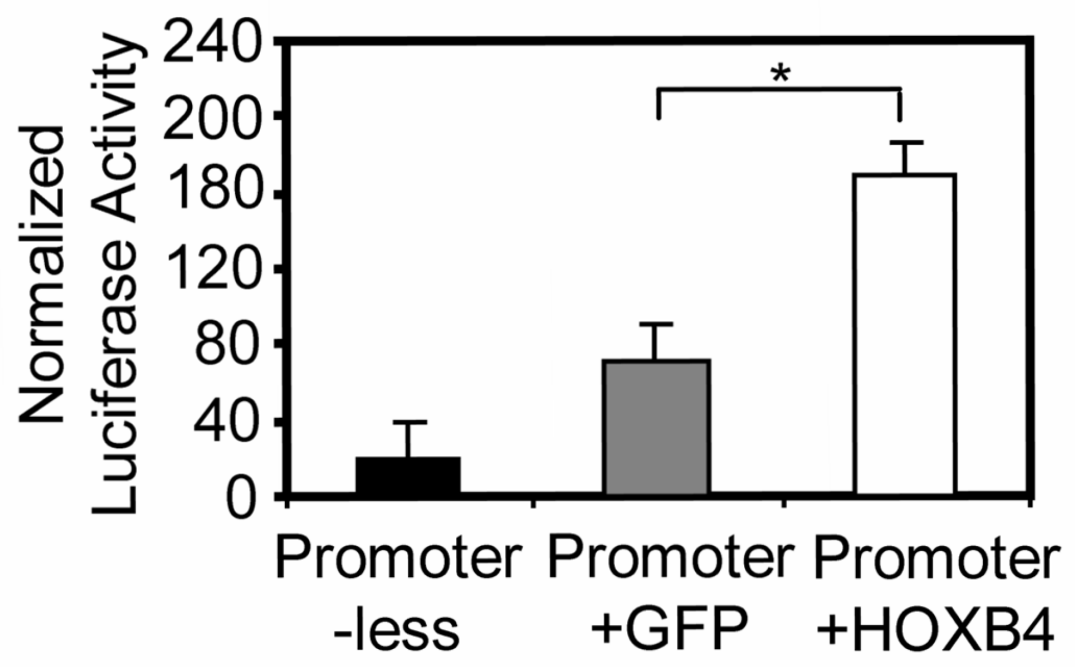

Figure 3-2. HOXB4-dependent increase in promoter activity of Hemgn in MEL cells. * $p<0.05$ 
binding. ${ }^{129}$ To directly test whether HOXB4 can bind to this site, we designed DNA oligonucleotide probes spanning this specific site $(-1562 b p \sim-1540 b p)$ to examine the binding of HOXB4 by using EMSA. Indeed, HOXB4 binding caused a band shift that was detected when the biotin-labeled wild type (WT) probe was incubated with purified GST-HOXB4 protein (Figure 3-3, lane 2). No band shiftwas detected in the reactions with a competitive unlabeled probe (lane 4), or a probe containing mutated "TAAT" core sequence (lane 6), or with control GST protein (lane 1, 3, 5, and 7). Addition of a HOXB4 antibody resulted in supershifting of the specifically retarded fragment (lane 8 ). These results demonstrate that HOXB4 can directly bind to site 1 in the promoter region of Hemgn in vitro. The limitation of this assay lies in the fact that excessive probes and purified HOXB4 protein were incubated together in vitro in reaction tube, and therefore do not directly test for in vivo occupancy of sites.

\subsubsection{HOXB4 Was Recruited to the Promoter Region of Hemgn In Vivo}

To discern whether HOXB4 can be recruited to the promoter region of Hemgn in bone marrow cells in vivo, we developed the first reported ChIP assay for HOXB4 binding in transduced bone marrow cells by using an anti-HOXB4 antibody (clone I12) that we first showed can be used to efficiently immunoprecipitate HOXB4 from a HOXB4-overexpressing cell line (Figure 3-4). The advantage of the ChIP assay is that the detected DNA-protein interaction occurs in the living cells in vivo instead of in artificial established in vitro system, faithfully reflecting the true biological process in a physiological microenvironment. Primary bone marrow cells were used here in which enforced expression of HOXB4 was shown to induce expansion of myeloid progenitor cells. PCR primers were designed to span several regions in the cis-regulatory elements of Hemgn that cantain each of the four putative HOXB4 binding sites.

Cellular extracts from transduced bone marrow cells were immunoprecipitated with the I12 antibody and DNA fragments of an average 500bp in size were released for PCR detection (Figure 3-5). We designed semi-quantitative PCR primer pairs to span the $-1568 \mathrm{bp} \sim-1447 \mathrm{bp}$ region containing 2 putative HOXB4 binding sites (site 1 and site 2, Figure 3-1) or to span the -543bp $\sim-228 b p$ region which contains 1 putative site (site 4, Figure 3-1). These two regions were specifically enriched by the I12 anti-HOXB4 antibody in the HOXB4-transduced bone marrow cells (Figure 3-6). In contrast, neither of these two regions was enriched in cells transduced with the GFP control vector, or in HOXB4 vectortransduced cells immunoprecipitated using an isotype control antibody. PCR primers amplifying an irrelevant region in Actb promoter containing no "TAAT" sequence showed no detectable enrichment with either antibody (Figure 3-6). These results verify that HOXB4 bound to the promoter of Hemgn in vivo in primary bone marrow cells that overexpressed HOXB4. 


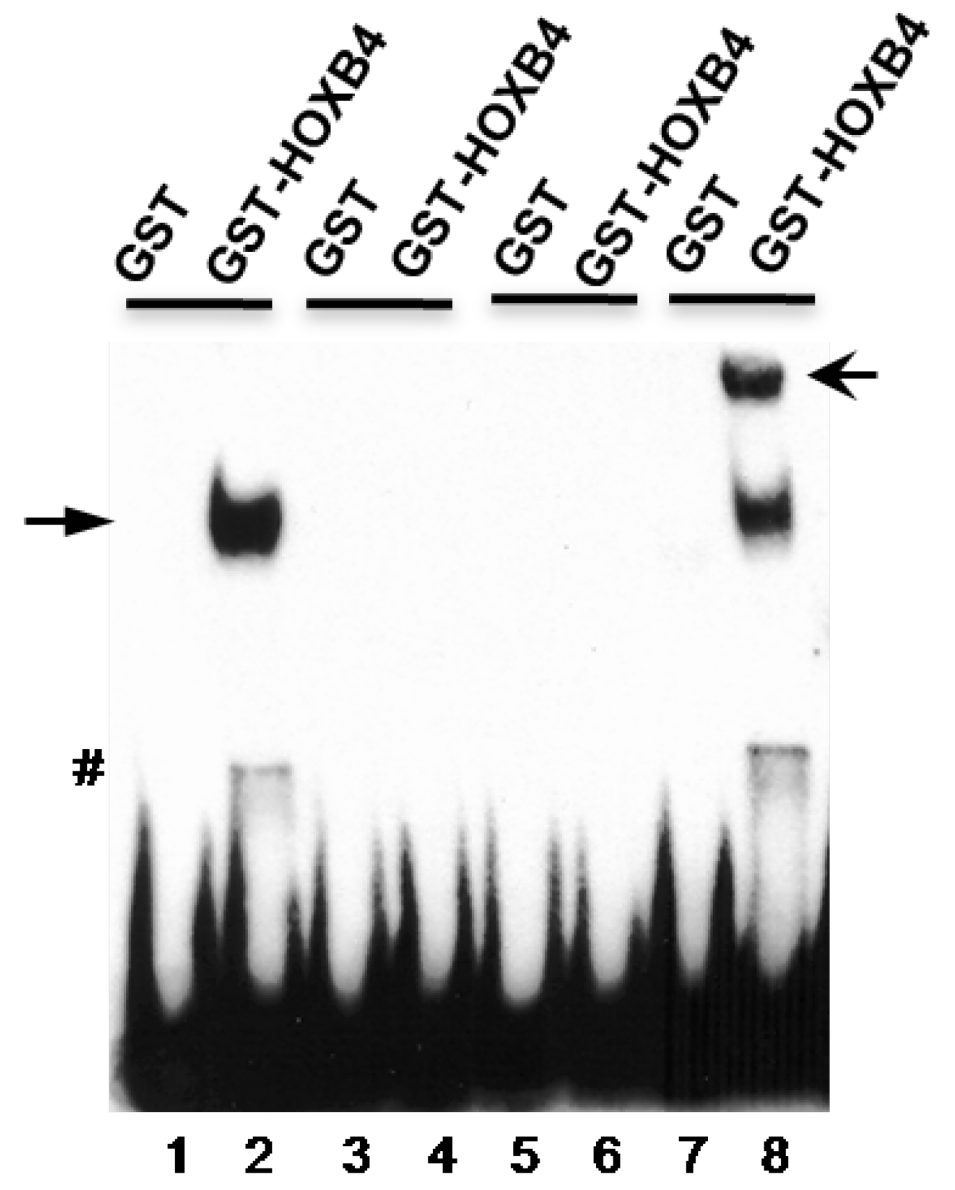

Figure 3-3. HOXB4-specific binding caused band shift of probes containing the putative HOXB4 binding site (site 1) in EMSA. 1-2: biotin-labeled WT probe (-1562 -1540bp of Hemgn promoter); 3-4: labeled WT probe: competitive unlabeled probe=1:200; 5-6: probe with "TAAT" core sequence mutated to "AGCA"; 7-8: labeled WT probe + anti HOXB4 antibody (I12); solid arrow: band shift by HOXB4; open arrow: supershift by I12; \#: degraded GST-HOXB4 caused band shift. 


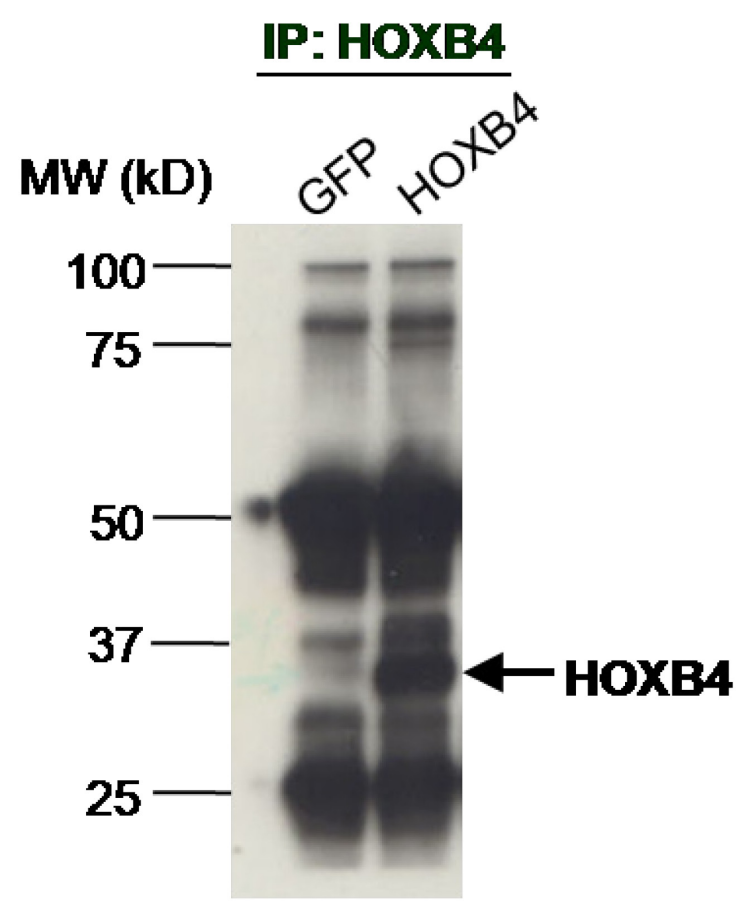

WB:HOXB4

Figure 3-4. Immunoprecipitation and western blotting detection of HOXB4 protein from GPE+86 cells overexpressed with GFP control or HOXB4 with monoclonal anti-HOXB4 antibody (I12). 


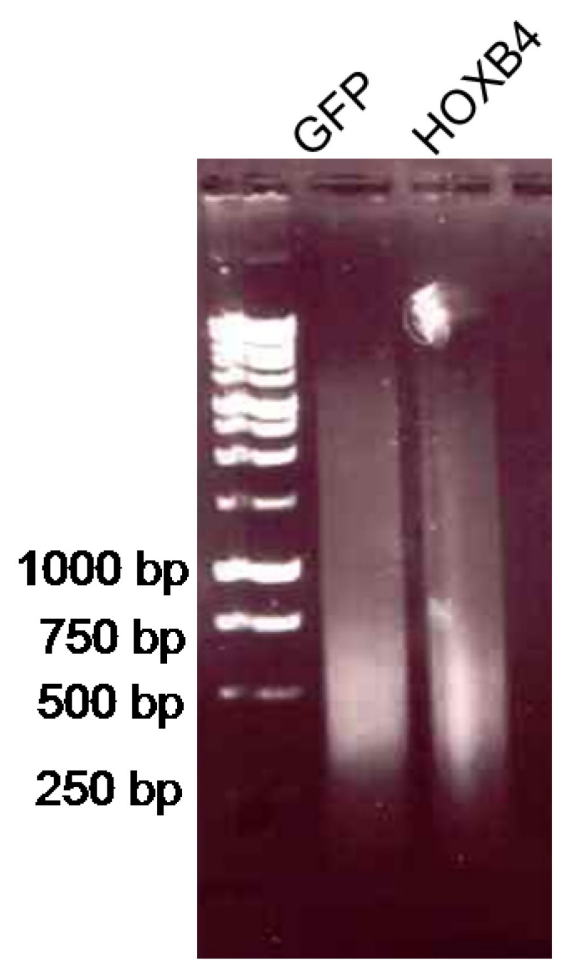

Figure 3-5. Sonicated chromatin fragments from GFP or HOXB4-transduced bone marrow cells for ChIP assay. 


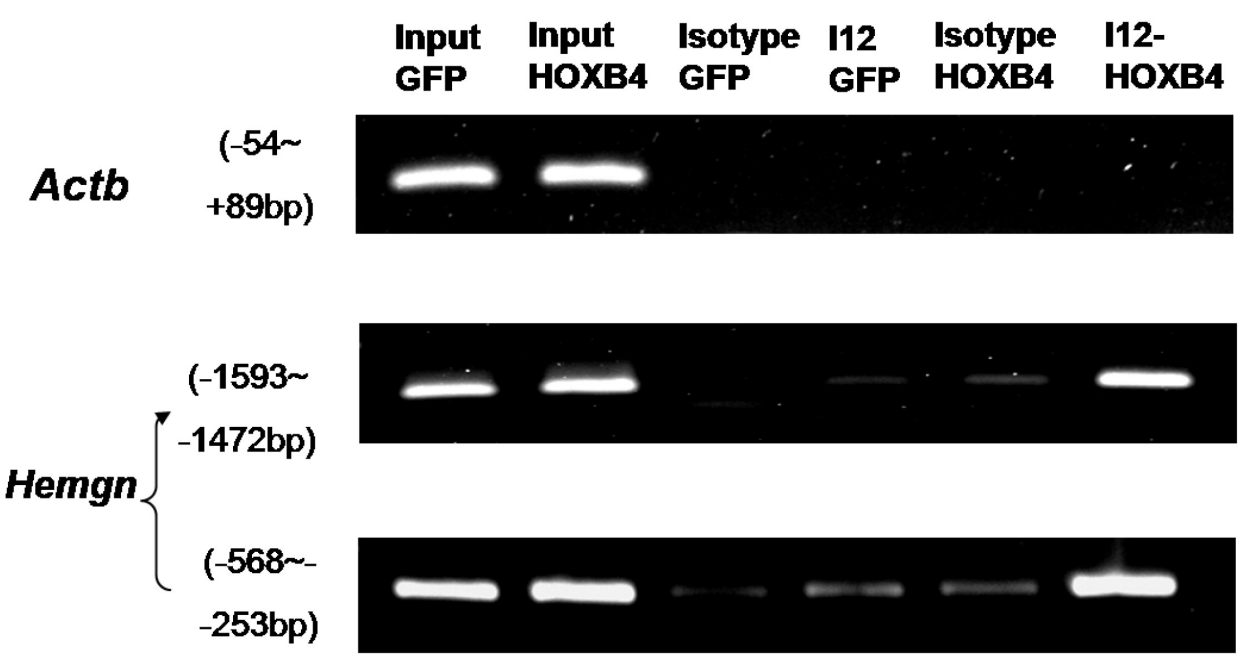

Figure 3-6. Semi-quantitative PCR detected enrichment of the promoter fragments of Hemgn by HOXB4 specific binding following ChIP assay in transduced bone marrow cells. 
To further quantify the fold enrichment of HOXB4 on the individual "TAAT" site, quantitative real-time PCR primers were designed to amplify the individual putative HOXB4 binding site in a 5' to 3' order in the promoter of Hemgn (Figure 3-7). The -1731bp -1542bp (site 1, Figure 3-1) and the -436bp -332bp (site 4, Figure 3-1) regions were enriched at relatively higher levels ( 16.1 fold and 34.8 fold). As sites 1, 2 and 3 are very close to each other within $500 \mathrm{bp}$, and due to the sonicated chromatin being around $500 \mathrm{bp}$, probably all the 4 sites contributed to direct binding by HOXB4.

\subsection{Discussion}

Classic microarray analysis for gene expression profiling does not provide definitive evidence of target gene relationships and needs to be combined with DNA binding data in order to identify direct target genes. In our study, the binding of the HOXB4 transcription factor to a target promoter has been measured using transient co-activation assays, EMSA, and ChIP assays. We selected a specific candidate target gene, called Hemgn, for these studies for the reasons discussed in Chapter 2, but this could in principle be done with any of the genes in our list.

Multiple "TAAT" core sequences with HOXB4-preferred flanking nucleotides were found within the $2 \mathrm{~kb}$ promoter region of Hemgn. As mentioned previously in Chapter 1, Hox regulation of downstream target genes occurs through binding to specific conserved DNA sequences in their regulatory elements. However, other Hox family members in addition to HOXB4 may also bind to the same "TAAT" core sequence through the homeodomain. Discrepancy exists between the relatively promiscuous binding of Hox monomers to DNA and the high specificity of Hox protein functions. It is hypothesized that the specificity of the downstream target regulation of Hox strongly depends on the flanking nucleotides surrounding the core sequence and on the cofactors which interact with Hox proteins to further increase the selective binding of Hox and cofactor heterdimers to DNA. However, prior studies have shown that one cofactor of HOXB4, Pbx, is not required, and even limits, HOXB4-induced expansion of hematopoietic stem and progenitor cells in vitro and in vivo. ${ }^{140 ; 168 ; 169}$ Thus, the binding specificity of HOXB4 to regulatory elements in its target genes are more likely dependent on the flanking DNA sequence surrounding the "TAAT" core. The binding affinity possibly could be enhanced with existence of multiple "TAAT" core sequence other than single one in the regulatory elements.

We proved that at least some of the "TAAT" containing promoter sequences of Hemgn can be transcriptionally activated by HOXB4 in vitro by luciferase reporter assays, implying that this promoter is regulated by HOXB4 in vivo. Furthermore, direct binding of purified HOXB4 to a promoter fragment of Hemgn was confirmed in vitro by EMSA. This finding suggests that the protein-DNA complex can be formed with purified GST-HOXB4 and that other cofactors of HOXB4 such as $\mathrm{Pbx}$ are not required for the direct binding of HOXB4 to these sequences. 


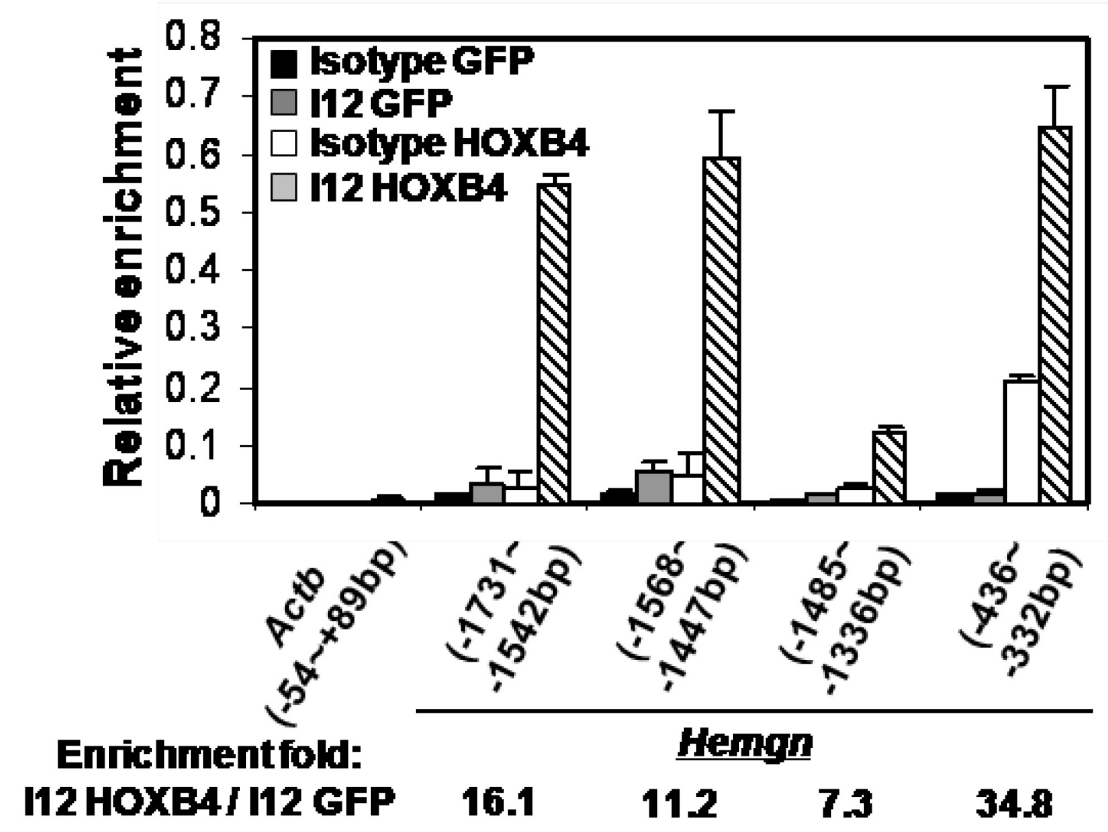

Figure 3-7. Quantitative real-time PCR detected relative enrichment of individual regions in Hemgn promoter by HOXB4 following ChIP assay in transduced bone marrow cells. All values were normalized to the corresponding input control sample. 
For the first time, we have established a ChIP assay for HOXB4 binding and show that Hemgn is indeed a direct target gene of HOXB4 in vivo. This validated in vivo HOXB4 binding region encompasses multiple "TAAT" sites identified in the promoter region of Hemgn. Intriguingly, each of the four "TAAT" sites in the promoter region of Hemgn was enriched to a similar extent in immunoprecipitated samples. This is possibly due to the fact that those "TAAT" sites are close in location, thus multiple "TAAT" sites within the 500bp sonicated chromatin fragments were immunoprecipitated and detected by PCR. It is necessary to mention again that when we mutated the "TAAT" core on site 1 which contains the most favored flanking nucleotides of HOXB4, the GST-HOXB4-DNA interaction was disrupted in vitro in EMSA. However, to determine exactly which site contributes most to the binding and downstream functional effects, site-mutagenesis assays could be performed in future studies.

In ChIP assays, we used constitutively overexpressed HOXB4 instead of inducible HOXB4-ER ${ }^{\top 2}$, based on the fact that although Hemgn is upregulated by HOXB4-ER ${ }^{\top 2}$ as early as 6 hours after TAM treatment, expression of Hemgn is also maintained at a high level in constitutively overexpressed HOXB4 samples by microarray (Brian P. Sorrentino's lab, data not shown). These data indicate that the direct regulation of Hemgn by HOXB4 is persistent after the initial binding and activation. 


\section{CHAPTER 4. EXPRESSION OF HEMGN EXPANDS BONE MARROW AND HEMATOPOIETIC PROGENITOR CELLS AND PROTECTS AGAINST APOPTOSIS}

\subsection{Summary}

To discern whether Hemgn is a downstream functional target of HOXB4, we constructed a MSCV retroviral vector for Hemgn overexpression. When overexpressed in 5-FU treated bone marrow cells, Hemgn promoted expansion of bone marrow cells and myeloid progenitor cells in in vitro liquid cultures and in secondary colony forming unit-culture (CFU-C) assays, recapitulating the effect of overexpressed HOXB4. Furthermore, both Hemgn and HOXB4 were antiapoptotic in transduced 5-FU treated bone marrow cells following cytokine withdrawal, suggesting a possible cellular mechanism for understanding the role of Hemgn in promoting expansion of primitive hematopoietic cells.

\subsection{Introduction}

Hemgn is a recently discovered nuclear protein that is expressed in testis and hematopoietic tissues. ${ }^{170 ; 171}$ In bone marrow, Hemgn is predominantly expressed in primitive progenitor and stem cell populations, but not in terminal differentiated mature blood cells. ${ }^{170}$ In transgenic mice in which EDAG, the human homolog of Hemgn, was driven by human CD11a promoter, enhancement of myelopoiesis and suppression of lymphopoiesis was found, suggesting a regulatory role in hematopoiesis. ${ }^{173}$ Since HOXB4 overexpression can cause bone marrow cell expansion during extended culture with myeloid cytokines, ${ }^{95}$ and because Hemgn is a direct transcriptional target of HOXB4 as discussed in Chapter 3, it is necessary to study the functional effects of Hemgn and whether Hemgn overexpression could recapitulate the expansion phenotype of HOXB4.

\subsection{Materials and Methods}

\subsubsection{Vector Construction}

The murine Hemgn cDNA (NCBI Reference Sequence: NM_053149.2) was cloned into Xhol sites in MSCV-IRES-GFP backbone including the following sequences from the 5' UTR:

5' AAGCTTTTAGGCTGCTTGTGAAGTCAAAGCCAATACCAAGAAGGCATCGT GGCAAG 3' and the 3' UTR: 5' CAGTGCTCAGT 3' (Figure 4-1). A polyclonal population of ecotropic retroviral producer GPE+86 cells ${ }^{179}$ was generated using a previously described approach. ${ }^{180 ; 181}$ Briefly, $2 \mu \mathrm{g}$ pCAGGS-VSVG and $4 \mu \mathrm{g}$ 
A

\begin{tabular}{lll|l|l|l|l|l|l|l|}
\hline MSCV LTR & HEMGN & MRES & GFP LTR \\
\hline
\end{tabular}

B

SUTR (56bp)

ORF:

AAGCTIITAGGCTGCTTGTGAAGTCAAAGCCAATACCAAGAAGGCATCGTGGCAAG ATGG CAGTGCTCAGT

Figure 4-1. Hemgn-expressing retroviral vector. (A) MSCV-retroviral vector construct with cDNA of Hemgn inserted upstream IRES cassette. (B) DNA sequence of the cloned 5'UTR together with 3'UTR of the CDNA of Hemgn in the MSCV vector. 
pEQ-PAM3(-E) helper plasmids were co-transfected with $4 \mu \mathrm{g}$ MSCV-cDNAIRES-GFP vector into $8 \times 10^{6}$ cycling 293T cells using FuGENE 6 reagent (Roche applied Science, Indianapolis, IN) according to the manufacturer's instructions. Retroviral vector supernatant were harvested 48 hours post transfection, pooled, filtered through $0.45 \mu \mathrm{m}$ PVDF filter (Millipore, Billerica, MA), and used for transducing the cycling GPE+86 cells in the present of $6 \mu \mathrm{g} / \mathrm{ml}$ polybrene. The transduced GFP positive GPE +86 cells were sorted and expanded in culture for establishing stable retroviral vector producer lines. The helper-free recombinant retroviral vector supernatants freshly collected from the GPE+86 producer cells were filtered through $0.45 \mu \mathrm{m}$ pore filter for transduction of bone marrow cells.

\subsubsection{Southern Blotting}

GPE+86 retroviral vector producer cells transduced with MSCV-IRES-GFP, MSCV-HOXB4-IRES-GFP, or MSCV-Hemgn-IRES-GFP vector were used in these assays. The genomic DNA extracted from the cells was digested with Sacl to release the cDNA-IRES-GFP fragment between the 2 LTRs of the MSCV-cDNA-IRES-GFP vectors. A probe targeting GFP was used for detecting the liberated fragment.

\subsubsection{Western Blotting}

Whole cell extracts were prepared from GPE +86 cells by M-PER Mammalian protein extraction reagent (Pierce, Thermo Scientific, Rockford, IL) supplemented with the protease inhibitors (Halt ${ }^{\mathrm{TM}}$ protease inhibitor cocktail kit, Thromo Scientific, Rockford, IL; Pefabloc SC Plus, Roche Applied Science, Indianapolis, IN). The proteins were then resolved on a $4-12 \%$ gradient NuPAGE Bis-Tris gel (Invitrogen) and transferred to $0.45 \mu \mathrm{m}$ PVDF membranes. The primary rabbit polyclonal IgG antibody for detecting Hemgn of mouse origin (M-180, sc-68360, Santa Cruz Biotechnology, INC, Santa Cruz, CA) was used for Hemgn detection.

\subsubsection{Transduction of 5-FU Treated Bone Marrow Cells}

The 5-FU treated bone marrow cells were prepared as described in Chapter 3. Briefly, bone marrow cells were harvested 4 days after intraperitoneal injection of mice with $150 \mathrm{mg} / \mathrm{kg} 5$-fluorouracil (5-FU) ${ }^{185 ; 186}$ from the tibias and femurs of 7 to 12 weeks old female $\mathrm{C} 57 \mathrm{BL}$ and6J mice (The Jackson Laboratory) as previously described. ${ }^{182}$ Red blood cells were lysed with BD pharm Lyse ${ }^{\mathrm{TM}}$ lysing buffer (BD Biosciences, San Jose, CA). The 5-FU treated bone marrow cells were prestimulated for 2 days with the cytokine-supplemented DMEM medium as described in previous Chapters. The cells were then spin-infected at $2000 \mathrm{rpm}$ for 30 minutes at room temperature by using plates preloaded with $25 \mu \mathrm{g} / \mathrm{ml}$ RetroNectin (Takara, Madison, $\mathrm{WI}$ ) and retroviral vector supernatant generated 
as described above at an multiplicity of infection (MOI) of 10 to 20 . After 3 to 4 rounds of transduction in 2 consecutive days, cells were collected, washed with PBS, and then resuspended in the cytokine-supplemented medium. Proportions of the transduced GFP positive cells were determined by flow cytometry.

\subsubsection{In Vitro Expansion Assays}

After retroviral vector transduction, the transduction efficiencies obtained were typically $30 \%$ for the GFP control and HOXB4 vectors, but around $13 \%$ for the Hemgn vector. For liquid culture and CFU-C assays, cells were mixed with nontransduced cells to have the same GFP percentage (13\%), and then equal numbers of normalized cells were cultured in cytokine-supplemented medium or M3434 methylcellulose medium as described previously. GFP percentage was determined by flow cytometry during the culture for evaluating growth advantage.

\subsubsection{Apoptosis and Cell Cycle Assays}

The transduced 5-FU treated bone marrow cells were cultured in the cytokinesupplemented Dulbecco's modified Eagle medium (DMEM) containing $20 \mathrm{ng} / \mathrm{ml}$ recombinant mouse IL-3, $50 \mathrm{ng} / \mathrm{ml}$ human IL-6, $50 \mathrm{ng} / \mathrm{ml}$ mouse SCF (R\&D Systems, Minneapolis, MN), 15\% FBS, 100 units $/ \mathrm{ml}$ penicillin, $100 \mu \mathrm{g} / \mathrm{ml}$ streptomycin and $2 \mathrm{mM}$ L-glutamine (R\&D Systems, Minneapolis, MN). After 6 days, cells were washed with PBS and cultured in the medium containing $1 \%$ FBS and no cytokines. Sixteen hours later, $3 \times 10^{5}$ cells were stained with Annexin $\mathrm{V}$-allophycocyanin (APC) (BD Biosciences, Franklin Lakes, NJ). Flow cytometry was used to detect GFP and APC percentage. For cell cycle analysis, the transduced cells were cultured for 6 and 16 days in the cytokine-supplemented medium and then were sorted to separate GFP positive and negative population, which were respectively stained with PI for DNA content analysis. The ModFit program (Verity Software House, Topsham, MI) was used to determine the percentage of cells at the $\mathrm{G}_{1}, \mathrm{~S}$, and $\mathrm{G}_{2}-\mathrm{M}$ phases of the cell cycle.

\subsection{Results}

\subsubsection{Expression of Hemgn in Transduced Cells}

In order to answer the question whether overexpression of Hemgn could contribute to the expansion phenotype of HOXB4, a full length cDNA of murine Hemgn ordered from ATCC was cloned into the MSCV-IRES-GFP retroviral vector. Integration and protein expression of Hemgn were confirmed in GPE+86 vector producer cells by southern and western blotting (Figure 4-2A and B). However, the GFP expression from the MSCV-Hemgn-IRES-GFP vector was 


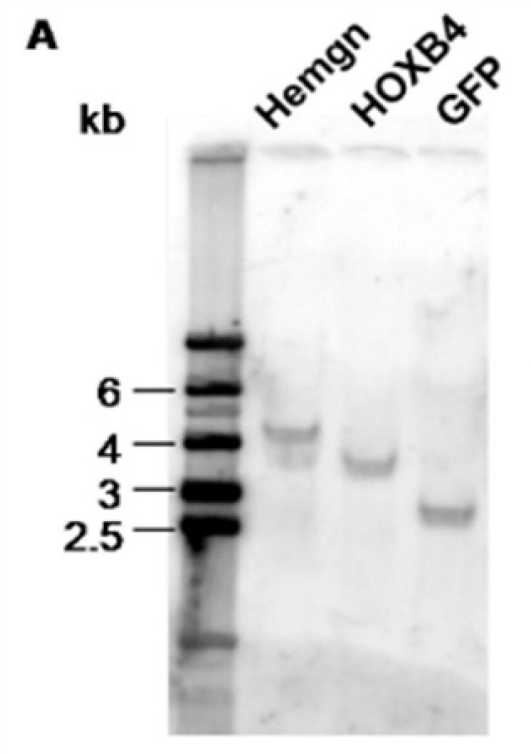

\section{B}

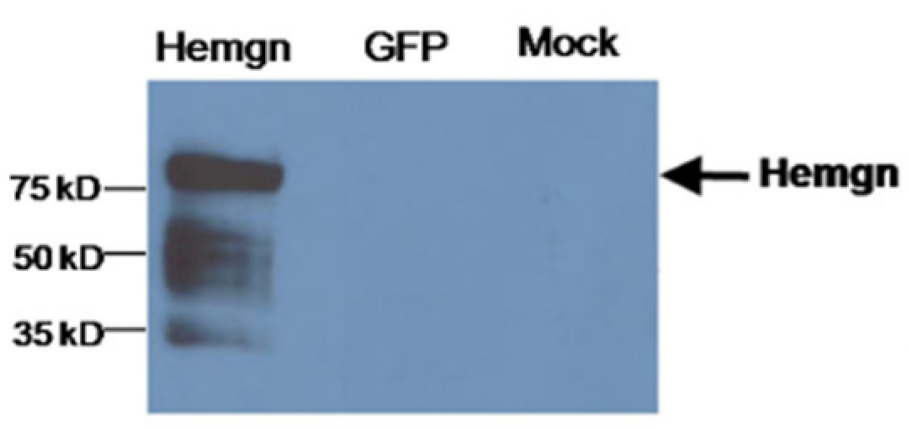

C

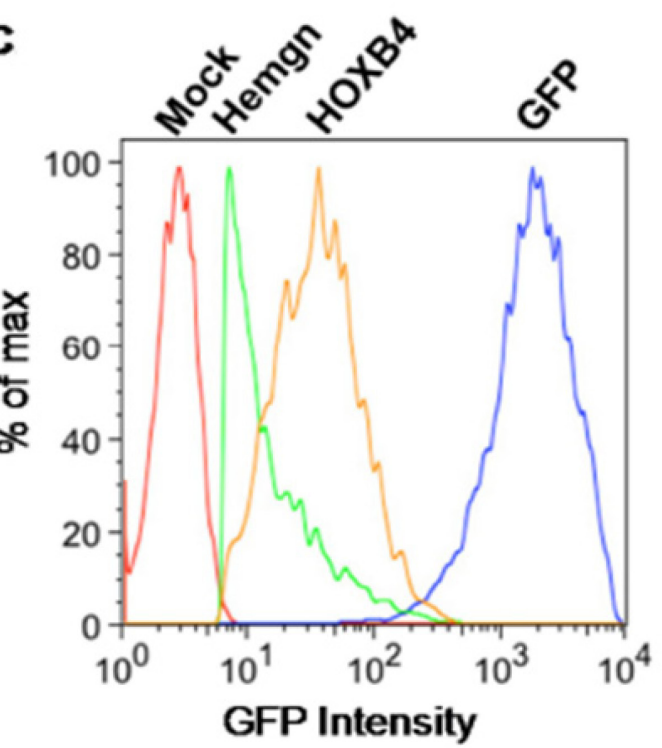

D

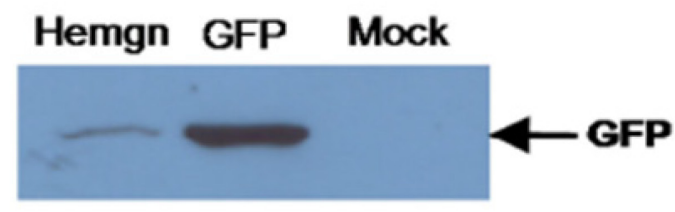

Figure 4-2. Integration, and protein expression of Hemgn and its GFP reporter in GPE+86 vector producer and bone marrow cells. (A) Southern blot detected the expected fragment size of integrated MSCV-cDNA-IRES-GFP vector: Hemgn vector-4547bp; HOXB4 vector-3576bp; GFP-only vector-2881bp. Enzyme: Sacl; Probe: GFP. (B) Western blot with polyclonal anti-Hemgn antibody (M-180) for Hemgn protein detection in GFP-only or Hemgn vectortransduced GPE+86 cells. (C) FACS analysis plot for GFP signal in bone marrow cells transduced with GFP control, Hemgn or HOXB4 vector. (D) Western blot detected GFP protein expression in GFP-only or Hemgn vector-transduced GPE +86 cells. Smear bands around 50kd and 35kd: degraded Hemgn. 
relatively low compared to control vectors as indicated by the mean fluorescence intensity for GFP signal in the transduced bone marrow cells (Figure 4-2C) and by the western blotting for GFP in the GPE+86 vector producer cells (Figure 4-2D). Furthermore, the titer of the MSCV-Hemgn-IRES-GFP vector was relatively low $\left(\sim 1 \times 10^{5}\right.$ virus $/ \mathrm{ml}$ compared to $\sim 1 \times 10^{6}$ virus $/ \mathrm{ml}$ from HOXB4 producer cells). These problems with the Hemgn vector might be due to instability of the vector coded mRNA or the complex structure of the inserted cDNA of Hemgn. This deficiency resulted in relatively low transduction efficiency when bone marrow cells were transduced with this vector supernatant.

\subsubsection{Hemgn Promoted Hematopoietic Cell Expansion in Liquid Culture}

Despite the problems with the MSCV-Hemgn-IRES-GFP vector, I next tested the effects of enforced overexpression of Hemgn in primary hematopoietic cells. 5 -FU was used to generate a hematopoietic progenitor cell-enriched population for vector transduction. Because the transduction efficiency of MSCV-HemgnIRES-GFP vector was $13 \%$, we diluted the GFP control and HOXB4overexpressing bone marrow cells with mock cells to the same proportion to examine whether there will be a growth advantage in subsequent liquid culture and CFU-C experiments (Figure 4-3).

We found that cells transduced with MSCV-Hemgn-IRES-GFP vector had a significant growth advantage over non-transduced cells in liquid culture, similar to the results seen with the HOXB4 vector (Figure 4-4). This was demonstrated by the increased GFP percentage of Hemgn-overexpressing and HOXB4overexpressing cells at day 6 (Hemgn: 31\%; HOXB4: 44\%), day 11 (Hemgn: 50\%; HOXB4: 78\%) and day 16 (Hemgn: 77\%; HOXB4: $91 \%$ ) from 3 biological independent experiments (Figure 4-4). In contrast, cells transduced with GFP control vector did not show any growth advantage during this time. These results show that, Hemgn and HOXB4 promote expansion of hematopoietic cells in liquid culture.

\subsubsection{Hemgn Promoted Myeloid Progenitor Cell Expansion In Vitro}

As HOXB4 overexpression can also cause dramatic expansion of myeloid

progenitor cells, ${ }^{95}$ we examined whether Hemgn has similar effect in CFU-C assays. The $13 \%$ cell mixtures were plated in semi solid medium and re-plated 7 days later to generate secondary CFU-C colonies. This assay allowed quantitation of myeloid progenitor expansion. We found that Hemgnoverexpressing bone marrow cells generated significiantly more secondary CFUC colonies than mock (4.9 fold) and the GFP control cells (6.1 fold) (Figure 4-5). As a positive control, the HOXB4-overexpressing bone marrow cells generated 7.9 fold and 9.9 fold more secondary colonies than mock and GFP control cells, respectively. To confirm that the detected increase of secondary CFU-C colonies 
7-12 weeks old C57BL/6 mice

5-FU 4 days
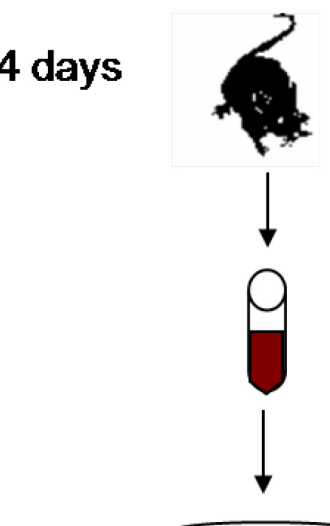

RBC lysed BM

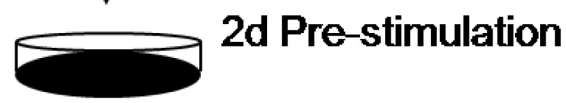

2d Transduction :

$\downarrow \quad$ Virus from GPE+86 producer

cells supernatant with

$\downarrow \mathrm{MOI}=10 \sim 20$

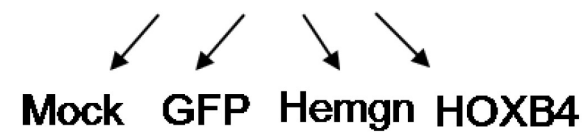

$\downarrow$

FACS: GFP analysis

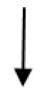

$13 \%$ GFP normalization with competitive mock cells

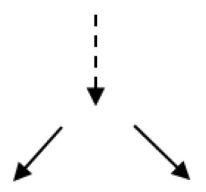

Competitive Liquid

Competitive $2^{\circ}$

Culture Assay

CFU assay

Figure 4-3. Experimental design of in vitro expansion assays. 


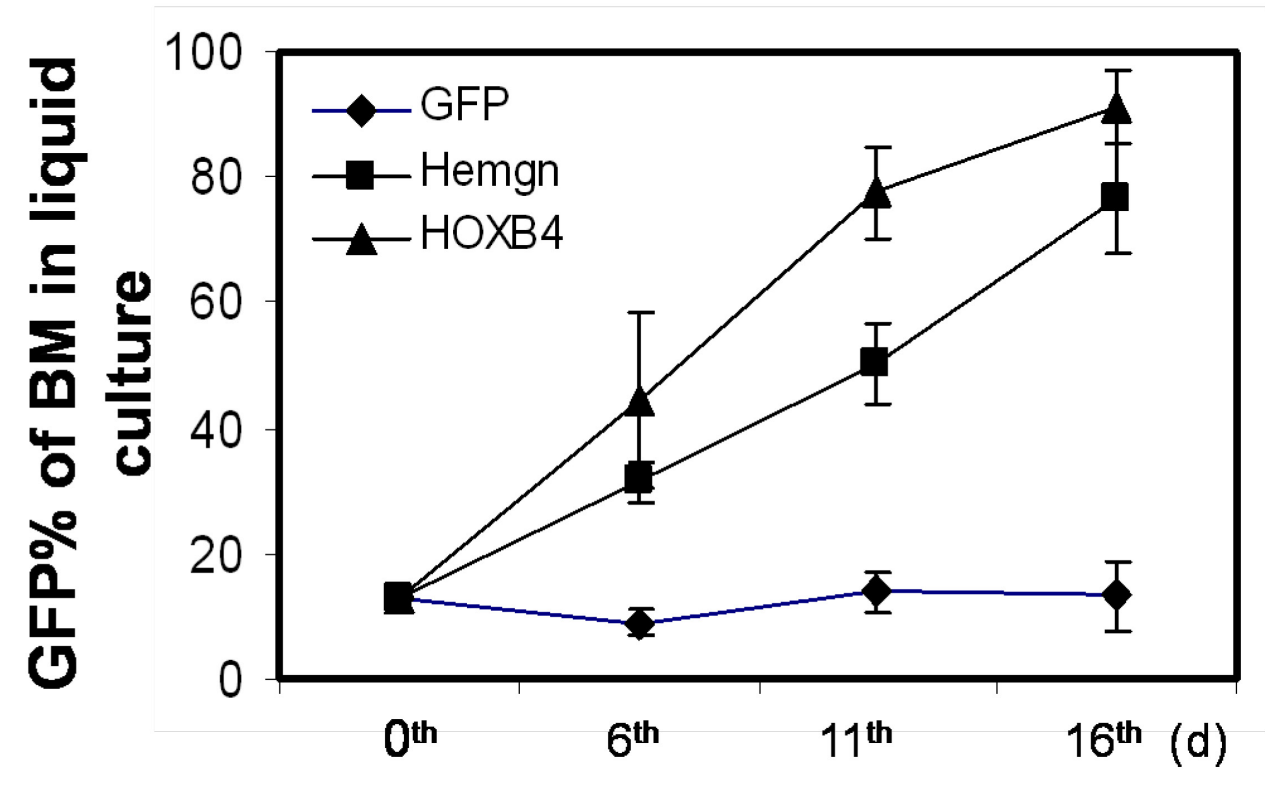

Figure 4-4. Growth advantage of Hemgn-overexpressing bone marrow cells in liquid culture. Flow cytometry-detected GFP percentage of the transduced bone marrow cells at $6^{\text {th }}, 11^{\text {th }}, 16^{\text {th }}$ day in liquid culture post-transduction. $0^{\text {th }}$ day: the day virus transduction was finished, when GFP percentage of each sample was normalized to $13 \%$ with mock cells. Three biological independent experiments were applied for statistics. 


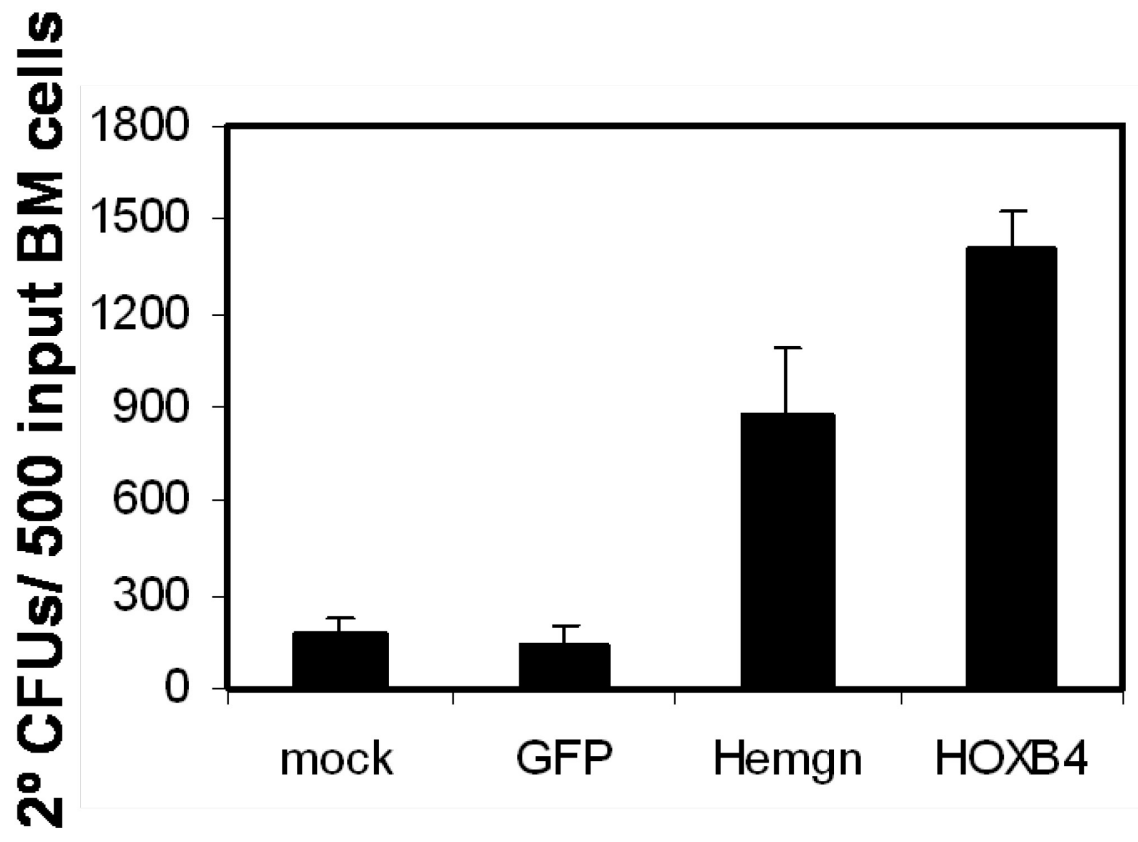

Figure 4-5. Growth advantage of Hemgn-overexpressing hematopoietic progenitor cells in methylcellulose culture. Secondary CFU-C colony number generated from the $13 \%$ GFP positive cell mixture. Three biological independent experiments were applied for statistics. 
were indeed caused by expansion of the initial sub-population of Hemgn or HOXB4-transduced bone marrow cells, the proportion of GFP positive cells were scored by flow cytometry both from primary and secondary CFU-C dishes (Figure 4-6). We found that in Hemgn-overexpressing cells, the GFP percentage increased from the pre-plating $13 \%$ to $32 \%$ in cells collected from primary colonies and to $69 \%$ in cells collected from secondary colonies, respectively (Figure 4-6). As a positive control, GFP percentage in HOXB4-overexpressing cells increased from the pre-plating $13 \%$ to $58 \%$ in primary colony cells and to $91 \%$ in secondary colony cells, respectively. GFP control bone marrow cells did not show any expansion advantage. This result reveals that, Hemgn and HOXB4 transduced cells had a selective advantage during growth both in primary and in secondary semi-solid CFU-C assays.

\subsubsection{Hemgn Protected Bone Marrow Cells from Apoptosis}

To explore the potential cellular mechanism of how Hemgn promotes expansion of bone marrow cells in vitro, we first examined apoptosis phenotypes in Hemgn-overexpressing bone marrow cells. Transduced bone marrow cells were cultured for 6 days in cytokine-supplemented DMEM medium containing 20

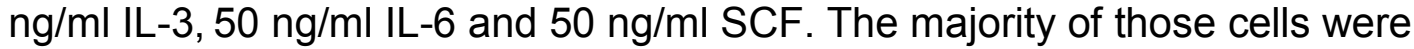
viable (89\%), and only a very small population was Annexin V+/ Propidium lodide(PI)- $(7 \%)$. The principle of this assay is based on the fact that the phosphotidylserine components of the phospholipid bilayers are externalized during the apoptotic process and can be detected by fluorescence labeled Annexin $\mathrm{V}$, which is a member of the annexin family of calcium-dependent phospholipid-binding proteins. Under these conditions in liquid culture, Hemgn and HOXB4 decreased the proportion of Annexin V+/PI- cells from $7 \%$ to $2 \%$ and $1 \%$ respectively (data not shown). In order to induce more cells to undergo apoptosis, we then transferred these cells into DMEM medium containing $1 \%$ FBS and no cytokines. Sixteen hours later, cells were stained for Annexin $V$ and PI. Flow cytometry was used to gate on the GFP positive and negative fractions for apoptosis detection.

A representative experiment (Figure 4-7) shows that both Hemgn and HOXB4 transduction resulted in a much smaller fraction of Annexin $\mathrm{V}+/ \mathrm{PI}$ - cells than seen in non-transduced cells or GFP control-transduced cells. Statistic analysis from 3 biologically-independent experiments (Figure 4-8) demonstrated that GFP control-transduced cells had a similar percentage of Annexin $\mathrm{V}+/ \mathrm{PI}$ - cells (45\%) as non-transduced cells $(43 \%)$ as expected. In contrast, HOXB4 transduction protected cells from apoptosis, demonstrated by the decrease of the percentage of Annexin V+/PI- cells in the transduced GFP positive population (3\%). Hemgn transduction also resulted in an anti-apoptosis effect as seen by gating on transduced cells ( $17 \%$ Annexin $\mathrm{V}+/ \mathrm{PI}-)$. This was significantly different than that seen with non-transduced GFP negative cells (53\% Annexin V+/PI-) (Figure 4-8). 


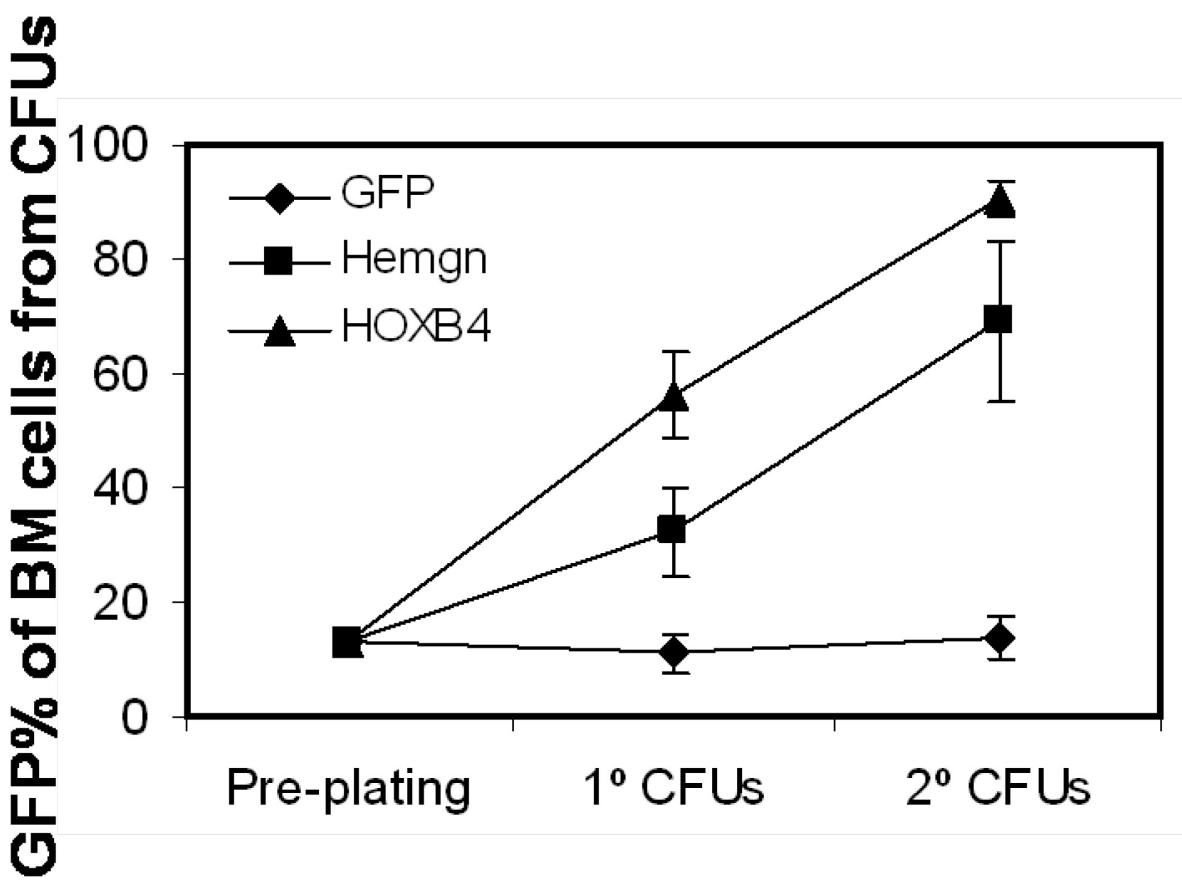

Figure 4-6. Flow cytometry-detected GFP percentage of bone marrow cells collected from primary and secondary CFU-C colonies. Three biological independent experiments were applied for statistics. 

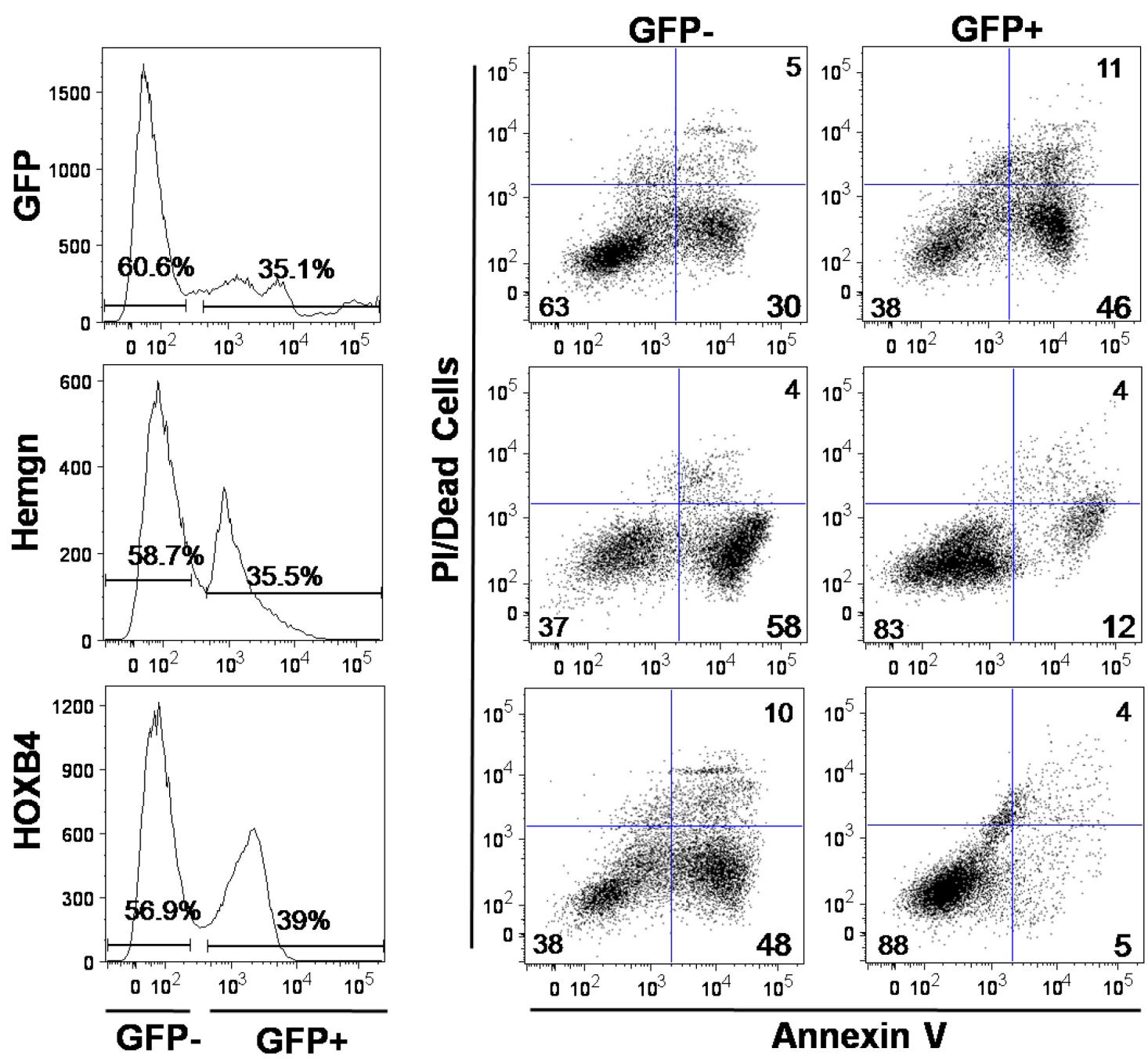

Annexin V

Figure 4-7. Flow cytometry analysis for apoptosis in GFP positive vs. negative population of transduced bone marrow cells in cytokine-depleted and serum-reduced medium. 


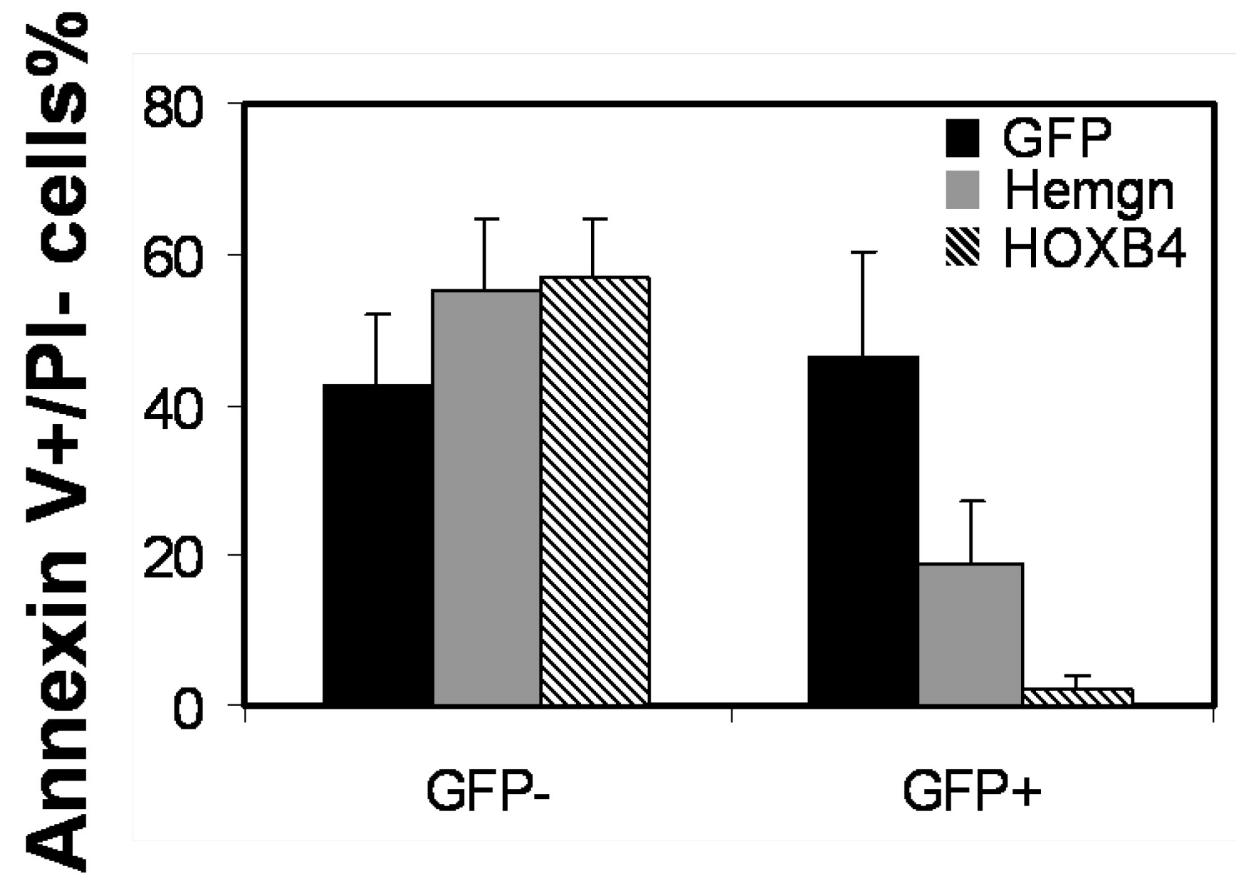

Figure 4-8. Quantification and statistics of three independent experiments for apoptosis in GFP positive vs. negative transduced bone marrow cells in cytokine-depleted and serum-reduced medium. 


\subsubsection{Hemgn Overexpression Has No Direct Effects on Cell Cycle in Hematopoietic Cells}

We have already shown that Hemgn can protect transduced cells from apoptosis, which could be one potential cellular mechanism of the way Hemgn promotes expansion of bone marrow cells in vitro. To explore another potential cellular mechanism, that is, whether Hemgn increases cell proliferation by altering cell division rates, cell cycle analyses were performed. For this assay, the transduced cells were cultured for 6 days in cytokine-supplemented medium and then were sorted to separate GFP positive and negative populations, which were then stained with PI for DNA content analysis. The percentage of cells at the $G_{1}, S$, and $G_{2}-M$ phases of the cell cycle was determined (Figure 4-9). We found that the percentage of cells in S phase was similar in Hemgn-transduced cells versus cells transduced with the control vector ( $38 \%$ and $42 \%$ respectively), indicating that the cell proliferation rate was not altered in Hemgn-overexpressed cells. The percentage of cells in $\mathrm{G}_{1}$ and $\mathrm{G}_{2}-\mathrm{M}$ phases were also not changed in Hemgn samples. The percentage of cells in S phase in the HOXB4overexpressing sample was slightly higher than the control $(49 \%$ and $42 \%$ respectively) (Figure 4-9), suggesting that HOXB4 could promote some cells to undergo division.

\subsection{Discussion}

Our results in overexpression studies show that Hemgn recapitulates the phenotype of HOXB4 in promoting expansion of bone marrow cells and myeloid progenitor cells in vitro. These results indicate that increased Hemgn expression may play a direct role in the mechanism by which HOXB4 induces myeloid progenitor expansion. Our results also suggest that Hemgn may play a role in hematopoietic stem cell self-renewal; however, transplant experiments will be required to prove this hypothesis.

Self-renewal that results in expansion of hematopoietic stem and progenitor cells requires the combination of both survival and proliferation signals together with the inhibition of apoptosis and differentiation. A large body of evidence suggests that suppression of apoptosis is required for hematopoietic stem cell survival and thus self-renewal. ${ }^{21 ; 22 ; 187 ; 188}$ EDAG has been reported to enhance survival of $\mathrm{Ba} / \mathrm{F} 3$ cells by protecting them from apoptosis following IL-3 withdrawal. ${ }^{172}$ HOXB4 has also been shown to protect bone marrow cells from the apoptosis-inducing effects of the TNF-a pathway. ${ }^{144}$ In this work, we have found that both Hemgn and HOXB4 can protect bone marrow cells from apoptosis in liquid culture, particularly following cytokine withdrawal, suggesting an anti-apoptotic role of Hemgn and HOXB4 that may be important for selfrenewal of myeloid progenitors and/or hematopoietic stem cells. 


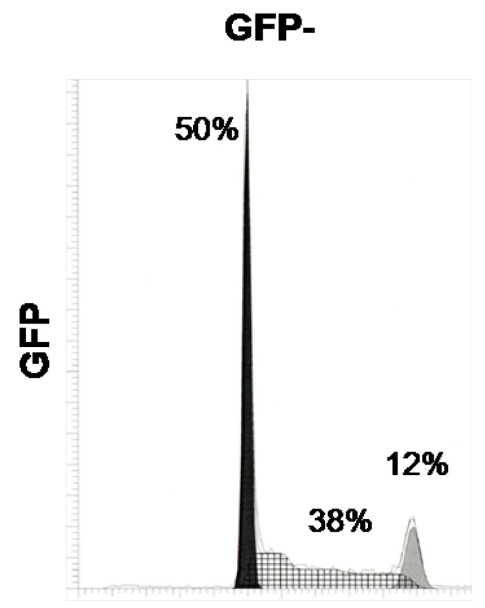

GFP+
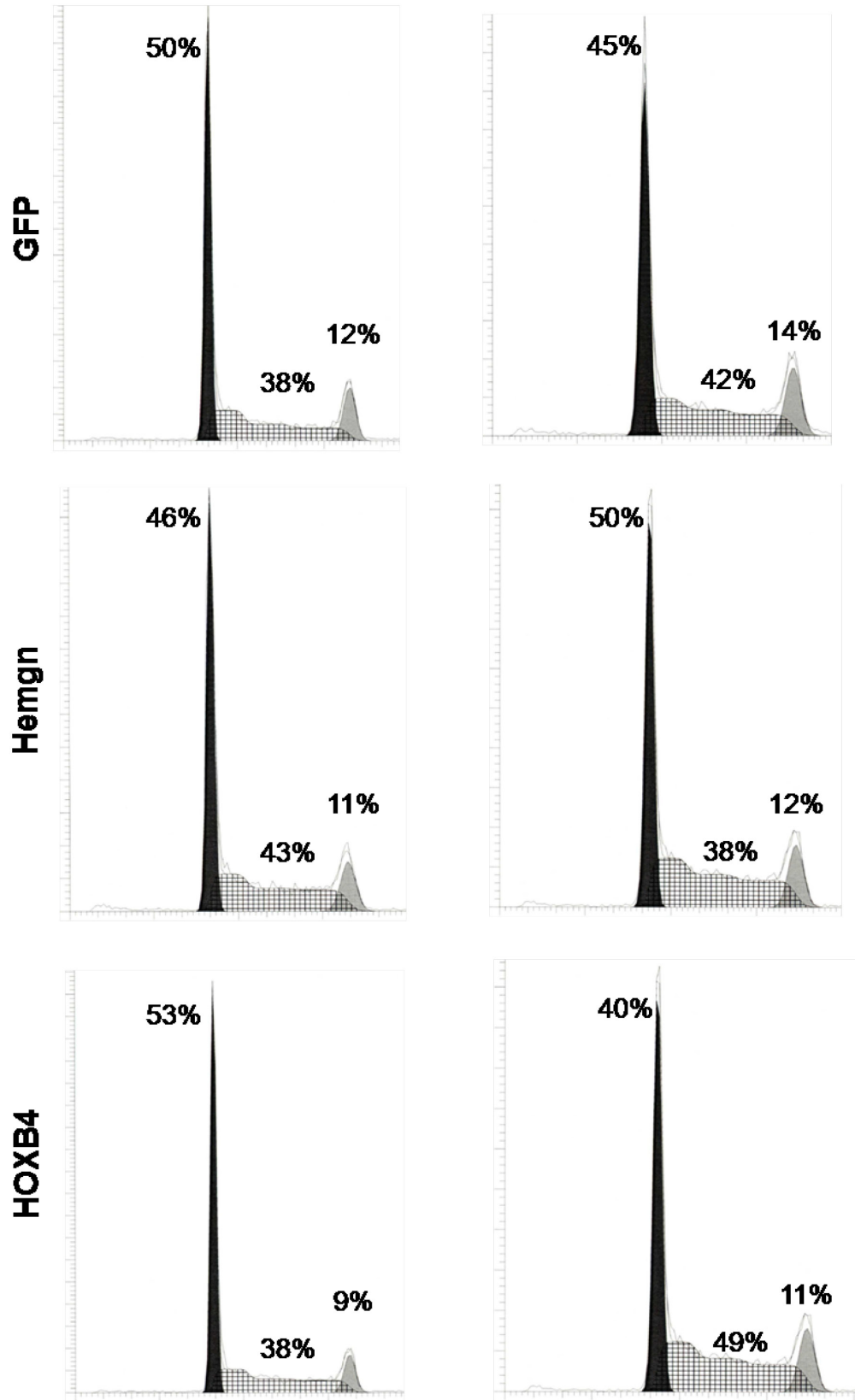

Figure 4-9. DNA content of vector-transduced bone marrow cells cultured for 6 days post transduction. DNA content was represented as the percentage of cells in $\mathrm{G}_{1}, \mathrm{~S}$ or $\mathrm{G}_{2}$ and $M$ phases. Left panel: non-transduced population; Right panel: transduced population; Black: $\mathrm{G}_{1}$ phase; Grid: $\mathrm{S}$ phase; $\mathrm{Grey}: \mathrm{G}_{2}$ and $\mathrm{M}$ phase. 
The cell cycle was not altered in Hemgn overexpressed cells, but was altered in HOXB4 overexpressed cells 6 days after transduction. These results suggest that the enhanced cell proliferation in HOXB4 samples was probably due to downstream events independent of Hemgn induction. We did observe an increased cell number in Hemgn and HOXB4 bone marrow samples compared to mock and vector control samples in the liquid culture assays. This is consistent with the result that the proportion of cells in S phase in Hemgn and HOXB4 samples was higher than GFP or mock control samples 16 days after transduction. As day 16 is a very late stage in the liquid culture, the observed consequence could be due to multiple causes. For example, Hemgn could block differentiation of cells in culture, resulting in enhanced cell number, a possibility suggested by the observation that EDAG overexpression can block the differentiation of HL-60 cells. ${ }^{172}$ There is also some evidence that ectopic HOXB4 expression can cause a delay in myeloid differentiation. ${ }^{154 ; 189}$

These in vitro results suggest further investigations to study whether Hemgn can cause expansion of hematopoietic stem cells in in vivo transplantation assays. We did transplant lethally irradiated recipient mice with donor-derived bone marrow cells transduced with Hemgn vector containing the GFP reporter gene. The reconstitution of GFP positive cells was poor in recipient mice 6 weeks post transplantation. The low titer and modest expression of the retroviral MSCV-Hemgn-IRES-GFP vector limited the ability to perform transplant assays. Further work is required to generate new lentiviral vectors for high and stable protein expression of Hemgn for use in transplant assays and to solve these technical issues. 


\section{CHAPTER 5. DISCUSSION}

\subsection{General Summary and Discussion}

\subsubsection{Target Genes Downstream of HOXB4}

By using the inducible HOXB4-ER ${ }^{\mathrm{T} 2}$ system, screening by expression microarray analysis identified a relatively small subset of potential downstream target genes $(n=77)$ of HOXB4. The high correlation of gene expression patterns obtained with the GFP control samples and the HOXB4-ER ${ }^{T 2}$ samples demonstrates no global change of gene expression following HOXB4 activation, thereby indicating that only a few key mediators exist downstream of HOXB4 and function in hematopoietic cell expansion. In eukaryotes, activation of gene expression involves an ordered assembly of transcriptional regulators, chromatinmodifying factors, RNA polymerase II, and associated general transcription factors onto cis-regulatory elements embedded in chromatin. Chromatinremodeling factors play an integral role in gene activation by perturbing chromatin structure and making specific loci permissive for transcription. Change of chromatin-remodeling factors usually causes the following global gene expression change. For example, knockdown of Bmi-1 and Mel-18, which belong to Polycomb group of transcriptional regulators, causes global changes in gene expression. ${ }^{190 ; 191}$ Our data indicates that epigenetic alterations and chromatin reorganization are not induced by HOXB4, at least at a global level. Thus, chromatin-remodeling factors are probably not direct targets of HOXB4. This inference is consistent with a recent study when HOXB4 was overexpressed. ${ }^{144}$

Of the 77 candidate target genes, 44 were upregulated and 33 were downregulated by HOXB4. This suggests that either gene activation or repression can be mediated by HOXB4, which is consistent with previous reports showing that HOX family members can activate or repress gene expression. ${ }^{141}$ The choice between activation and repression of target genes by HOXB4 is not well-understood and is probably context specific. To distinguish the transactivation activity of HOXB4 from its repressive function, an engrailed repression domain-fused HOXB4 was introduced into hematopoietic stem and progenitor cells and was shown to completely block their expansion. ${ }^{95}$ In contrast, a VP16 activation domain-HOXB4 fusion protein was fully active in conferring expansion, suggesting the requirement of trans-activation function, but the dispensability of repressive function, of HOXB4 in hematopoietic stem and progenitor cell expansion. Therefore, the genes that were downregulated by HOXB4 in our microarray analysis may be less functionally relevant to the biological activity of HOXB4 in stem cell expansion. On the other hand, it is still possible that some of the repressive events are important. For example, Ms4a4c (membrane-spanning 4-domains, subfamily A, member $4 \mathrm{C}$ ), a trans-membrane protein that is expressed in hematopoietic stem and progenitor cells with unknown function, 
was downregulated following HOXB4 activation. When it was overexpressed in bone marrow cells through retroviral vector-mediated gene transfer, Ms4a4c robustly suppressed secondary CFU-C colony formation compared to the mock and the empty vector controls (Jie Jiang, unpublished data). These data indicate that downregulation of Ms4a4c could be involved in HOXB4-mediated expansion of hematopoietic progenitor cells. To test this hypothesis, one could knockdown Ms4a4c expression in the hematopoietic stem and progenitor cell through shRNA to investigate its effect on hematopoietic stem and progenitor cell expansion.

\subsubsection{The Mechanism of Hemgn as a Functional Direct Target of HOXB4}

We show that when overexpressed, Hemgn can partially recapitulate the phenotype of HOXB4 in promoting the expansion of bone marrow cells and myeloid progenitor cells in in vitro assays. These results suggest that Hemgn can potentially play a mechanistic role in HOXB4-induced myeloid progenitor expansion. The mechanism of Hemgn as a functional target of HOXB4 is most likely due to the fact that Hemgn can protect cells from apoptosis. This antiapoptotic effect was most apparent when the three cytokines: SCF, IL-3 and IL-6 were withdrawn and serum was reduced from culture medium, which is a common assay for inducing apoptosis in hematopoietic cells. However, we did note that the Hemgn overexpression phenotype only partially recapitulated the HOXB4 overexpression phenotype. Here I propose several explanations for this discrepancy.

First, retroviral vector-mediated expression of Hemgn usually results in high level expression in bone marrow cells due to strong retroviral promoter. In contrast, the mRNA level of Hemgn was increased merely 2 to 5 fold by HOXB4 overexpression. It is possible that different levels of transgene expression can result in quantitative or even qualitative differences in phenotype. For instance, the enforced expression of Hemgn may cause cytotoxicity which diminishes myeloid expansion and therefore results in a net effect that only partially resembles HOXB4. I hypothesize that a threshold of Hemgn expression level exists for optimal expansion of hematopoietic progenitor cells, like the case of HOXB4 itself. ${ }^{153}$ When Hemgn is expressed at a high level, the toxic effects of Hemgn may inhibit further expansion of hematopoietic progenitor cells. To test this hypothesis, overexpressing Hemgn in different doses using retroviral vectors or generation of a TAT-Hemgn fusion protein could be applied to detect such a threshold.

The second explanation of the functional difference between HOXB4 and Hemgn is that other target genes regulated by HOXB4 are likely to be corequired for the maximal effect of HOXB4. In support of this notion, we found that although Hemgn can protect bone marrow cells from apoptosis like HOXB4, it cannot promote $S$ phase entry and proliferation compared to the cells transduced by HOXB4. Thus, there probably are other target genes of HOXB4 that contribute 
to the expansion phenotype by promoting cell division rate. To test this hypothesis, one could overexpress Hemgn together with other direct targets of HOXB4 in bone marrow cells. In detail, one could transduce bone marrow cells with both MSCV-Hemgn-IRES-GFP and MSCV-new target cDNA-IRES-RFP retroviral vectors. The GFP and RFP double positive cells could then be sorted as a source of cells transduced with both genes for futher functional studies to test whether overexpression of both genes can fully recapitulate the phenotype of HOXB4 overexpression, particularly on its effects in both anti-apoptosis and cell cycle progression.

\subsubsection{Relationship between Hemgn Expression and Endogenous Hoxb4}

Our results showed that the endogenous Hemgn mRNA was increased about 2 to 5 fold following HOXB4 activation in expanded Lin ${ }^{-}$bone marrow cells. Furthermore, the exogenous expression of Hemgn induces expansion of murine bone marrow and myeloid progenitor cells in vitro. One important question suggested by my results is "What is the normal function of Hemgn?". The expression of Hemgn is restricted to the testis and hematopoietic tissues. ${ }^{170 ; 171}$ This pattern of expression is similar to Hoxb4 in hematopoietic tissues, with a spatial and temporal pattern in hematopoietic sites during embryogenesis. Hemgn is first expressed in blood islands of yolk sac, then in fetal liver, and finally in the adult spleen and bone marrow. ${ }^{170}$ In adult bone marrow, Hemgn is predominantly expressed in developmentally primitive stem and progenitor populations, and its expression decreases during differentiation. ${ }^{170}$ These facts are consistent with the hypothesis that endogenous Hemgn may play a role in regulating normal hematopoiesis. To address this question, we are generating mice in which both alleles of Hemgn are disrupted to test its role in development, particularly hematopoiesis.

To understand whether the expression of Hemgn can be regulated by endogenous Hoxb4, we performed quantitative real-time PCR assay in Hoxb4deficient bone marrow cells ${ }^{158}$ and detected a 5 -fold decrease in Hemgn mRNA level in c-kit ${ }^{+}$Scal- $1^{+} \mathrm{Lin}^{-}$cells when compared to wild type cells. These results suggest that Hemgn may also be regulated by endogenous Hoxb4 as opposed to overexpression constructs. However, the detectable expression of Hemgn in the absence of Hoxb4 indicates the presence of some degree of Hoxb4-independent regulation of Hemgn expression. One possibility is that other HOX4 paralog members could provide redundant function through upregulating Hemgn expression.

It is important to emphasize that, in this study, we were interested in defining the targets in the context of overexpressing an ectopic HOXB4 gene in mice, given that this is the biological setting in which expansion of hematopoietic progenitor cells have been noted. We recognize that these data may not necessarily represent target genes of endogenous Hoxb4 that is expressed at 
significantly lower levels. To further explore the genes regulated by endogenous Hoxb4, we could use microarray analysis to compare the gene expression difference in c-kit ${ }^{+}$Scall $-1^{+}$Lin $^{-}$cells between wild type and Hoxb ${ }^{-1-}$ mice. The disadvantage of this system is that the activity of Hoxb4 could not be temporally induced and thus the candidate list may not represent immediate early response genes. To overcome this disadvantage, one could replace the endogenous Hoxb4 gene with Hoxb4-ER ${ }^{T 2}$ fusion gene. Hoxb4-ER ${ }^{T 2}$ is inactive unless the mice are treated with tamoxifen, which can temporally induce Hoxb4-ER ${ }^{T 2}$ activation. Microarray analysis can be applied to compare the gene expression profile in c-kit ${ }^{+}$Scal- $1^{+}{ }^{+}$in ${ }^{-}$cells generated from tamoxifen treated Hoxb4-ER ${ }^{\top 2}$ mice and from non-treated Hoxb4-ER ${ }^{T 2}$ mice to identify the early response genes regulated by Hoxb4.

\subsubsection{Upstream Regulation of Hemgn: Other Mechanisms}

Although HOXB4 was proved to be recruited to its consensus binding motifs in Hemgn promoter by ChIP assay in hematopoietic progenitor cells in our study, it does not exclude the possibility of involvement of other regulatory elements of Hemgn. HOXB4 could regulate Hemgn expression by recruiting other hematopoietic regulators that bind directly or indirectly to the Hemgn promoter. Alternatively, the recruitment of other transcription factors could be HOXB4independent but could be necessary for maximal HOXB4 activity. The Hemgn promoter contains binding sites of multiple transcription factors in addition to HOXB4, including GATA-1, AML-1, Evi-1 and ETS. ${ }^{184}$ HOXB4 may directly interact with AML-1, Evi-1 or ETS proteins. To test this hypothesis, one could perform co-immunoprecipitation assay using HOXB4 antibodies. If a positive interaction partner can be found, one can map the interaction domain and test its function in HOXB4-mediated hematopoietic cell expansion.

GATA-1, a member of the GATA family of transcription factors, is expressed in primitive and definitive erythroid cells, megakaryocytes, eosinophils, and mast cells. It is essential for normal erythroid and megakaryocytic development. GATA1 was shown to be recruited to the promoter of Hemgn in an AML cell line, and to transactivate HEMGN in K562 cells. ${ }^{184}$ Gata $1^{-/}$mice die during early embryogenesis due to severe anemia with erythroid differentiation block at the proerythroblast stage and rapid apoptosis of those cells. Intriguingly, human HEMGN protects K562 cells from apoptosis and prevents their differentiation. Therefore, Hemgn may play a positive role in cell survival and a negative role in erythroid terminal differentiation as a consequence of GATA-1 upregulation in erythroid cells.

AML1 (Acute myeloid leukemia 1) is a transcription factor essential for hematopoiesis. AML1 gene is the most common target of chromosomal translocations in human leukemias. AML1-Evi-1 fusion protein can transform hematopoietic stem cells. ${ }^{192} \mathrm{AML} 1$ binds to sequences present in the regulatory 
regions of a number of hematopoiesis-specific genes, including certain cytokines such as GM-CSF (granulocyte macrophage colony-stimulating factor). ${ }^{193}$ Evi-1 has been recognized as a dominant oncogene associated with murine and human myeloid leukemia. It is a common and essential regulator for proliferation of embryonic and adult hematopoietic stem cells and transformed leukemic cells. ${ }^{194}$ Members of the ETS family have been implicated in a wide variety of functions including the regulation of cellular differentiation, cell cycle control, cell migration, cell proliferation, apoptosis and angiogenesis. Multiple ETS factors have been found to be associated with cancer through gene fusion. These transcription factors which show potential binding sites in the promoter of Hemgn possibly provide other upstream signals in regulating Hemgn expression.

\subsection{Short-Term Future Directions}

Although overexpression of Hemgn partially recapitulated the effect of HOXB4 in mediating hematopoietic progenitor cell expansion in vitro, it is unknown whether Hemgn upregulation by HOXB4 is required for the observed hematopoietic stem and progenitor cell expansion. To complement our studies, we will use short hairpin RNA (shRNA) to knockdown endogenous Hemgn in cells overexpressing HOXB4 to determine if this will lead to reduced hematopoietic stem and progenitor cell expansion. We have designed a set of different shRNA sequences targeting different regions of Hemgn, including the cDNA coding region and 3' UTR. Those shRNA nucleotides were individually cloned into a lentiviral vector containing the MSCV promoter. Lentiviral vector supernatant will be collected from transiently transfected 293T cells and used to transduce GPE +86 cells that constitutively overexpress exogenous Hemgn. The knockdown efficiency of different shRNAs will be tested by western blotting using an anti-Hemgn antibody. Preliminary experiments have identified at least two of the short hairpin RNAs that efficiently knockdown the protein level of exogenous Hemgn. We will transduce bone marrow cells with both the Hemgn-shRNA lentiviral vector and the MSCV-HOXB4-IRES-YFP vector. The cells successfully transduced by both vectors can be sorted for functional assays to evaluate the effects of Hemgn deficiency on HOXB4-induced hematopoietic progenitor cell expansion.

The disadvantages of any knockdown strategy are the potential off-target effects of the short hairpins and the inability to fully suppress target protein expression. To circumvent these drawbacks, generation of Hemgn-null mice by targeted gene disruption would be the optimal strategy and is ongoing. To study the physiological role of Hemgn in hematopoietic tissues, and to avoid the potential failure of germline transmission due to the lack of Hemgn, we have generated a conditional allele of Hemgn flanked by loxP sites, which can be removed through a hematopoietic tissue specific Cre (MMTV-Cre) during development or an inducible Cre (Mx1-Cre) in adult mice. The effects of Hemgn deficiency on hematopoiesis, particularly on hematopoietic stem and progenitor 
cells expansion, will be carefully investigated. We will perform liquid culture and CFU-C assays in vitro and competitive bone marrow transplantation in vivo. If the endogenous Hemgn is critical to hematopoietic stem cell function, I might expect to see a defect in self-renewal of Hemgn-deficient hematopoietic stem and progenitor cells. To further explore whether Hemgn is a key target of HOXB4, we can overexpress HOXB4 in Hemgn-deficient cells and assess its biological consequence similarly as I mentioned before.

\subsection{Long-Term Future Directions}

\subsubsection{Biochemical and Functional Characterization of Hemgn}

Hemgn is a newly identified protein and its biochemical and functional characterization is far from well-understood. A nuclear-localization signal together with a coiled-coil domain, but no other recognizable motifs, is present in the sequence of Hemgn. ${ }^{170}$ Careful characterization of this protein at biochemical level will be important for future studies. The lack of a DNA-binding domain reasonably lowers the possibility that Hemgn is a transcription factor. We will fuse Hemgn with a known DNA-binding motif and test its ability to activate or repress reporter gene expression. If we see no activity of this fusion protein in regulating gene expression, it would suggest that Hemgn has no transactivation domain by itself. Otherwise, if we see some activity of this fusion protein in regulating reporter gene expression, it would suggest that Hemgn may function as a cofactor that can recruit other transcriptional regulators through direct interaction. If the second situation occurs, it will be interesting to identify the interaction partners of Hemgn. Since coiled-coil proteins usually interact with other coiled-coil proteins for protein-protein recognitions and interactions, the present coiled-coil domain in Hemgn should play an essential role in interacting with other proteins for conveying the function of Hemgn. We can first make a deletion mutation in the coiled-coil domain of Hemgn. I expect to see a loss of Hemgn function in promoting hematopoietic progenitor cell expansion and in protecting cells from apoptosis when its coiled-coil domain is deleted. If this is the case, I will immuoprecipitate Hemgn in cells overexpressing wild type Hemgn or mutant Hemgn with an anti-Hemgn antibody and then perform proteomics analysis in order to identify its interaction partners. Since human HEMGN was shown to regulate the expression of some key genes in hematopoietic stem cell function, ${ }^{173}$ mouse Hemgn probably also acts as a transcription regulator through interaction with other transcription factors. Thus, microarray analysis can be conducted using wild type Hemgn and mutant Hemgn-overexpressing cells to identify differentially expressed genes. For example, I expect to see expression changes of genes that are involved in apoptotic pathways due to the fact that Hemgn shows profound effect in protecting bone marrow cells from apoptosis. 


\subsubsection{Potential Clinical Use of Hemgn}

Successful ex vivo hematopoietic stem cell expansion has been a long-sought goal in hematopoietic stem cell transplantation and hematopoietic stem cell gene therapy. Hemgn could potentially be used to achieve this goal. Since constitutive expression of Hemgn by retroviral vectors may have unforeseen side effects, such as insertional mutagenesis, transient exposure of hematopoietic stem cells to Hemgn may offer a better approach. Recently, progress has been made to directly deliver proteins into cells by fusing the protein with plasma membrane penetrating peptide sequences. Several proteins and peptides are able to pass through plasma membranes independent of classical receptor- or endocytosismediated process. An example is human immunodeficiency virus (HIV)-1 transactivator of transcription (TAT) protein. The small protein transduction domain of TAT was fused to HOXB4 and was used to deliver TAT-HOXB4 protein into bone marrow cells. ${ }^{195}$ The TAT-HOXB4 protein is fully active in supporting hematopoietic stem and progenitor cells expansion when added into the culture medium. ${ }^{195}$ This process of TAT-based transportation is dependent on heparan sulfate proteoglycan on the cell surface. The advantage of using this strategy is to avoid conventional gene transfer approaches, which could be tumorigenic due to gene integration into the genome. Furthermore, the TATbased protein transportation process is rapid, efficient and the dose is easy to control. Since Hemgn could have dose-dependent effects or side effects due to continuous constitutive expression as I discussed above, using a TAT-Hemgn fusion protein would be a promising approach to determine the optimal dose of TAT-Hemgn to mediate hematopoietic cell expansion in vitro. However, the biological activity of TAT-Hemgn should be determined first because TAT-Hemgn could potentially be inactivated when penetrating the plasma membrane, a phenomena that has been seen in some TAT-fusion proteins.

\subsubsection{Identification of Other Direct Target Genes of HOXB4}

The partial recapitulation of HOXB4 mediated hematopoietic stem and progenitor cell expansion by overexpression of Hemgn may suggest either that some other targets we identified using our gene expression profile approach, or some yet to be identified new targets are necessary for fully executing the HOXB4 downstream activity. In addition to studying other candidate genes in the generated 77 target list using the similar methods as we used before, other strategies such as ChIP-seq (Chromatin immunoprecipitation followed by sequencing) assay could be used to complement our microarray data. By using ChIP-seq, we can obtain genome-wide profiling of HOXB4 binding sequences. ChIP-seq offers higher resolution, less noise and greater coverage than its array-based predecessor ChIP-chip, which necessarily introduces some bias, as an array is restricted to a fixed number of probes. ${ }^{196}$ Another important advantage of ChIP-seq over the expression array is that only a small amount of DNA (10-50 ng) is required for generating reliable signal, ${ }^{196}$ which would allow us 
to use a purified pool of hematopoietic stem cells, instead of a mixed population of hematopoietic progenitor cells to obtain enough DNA sample.

\subsection{Detailed Consideration of These Results and Their Impact on Understanding the Molecular Mechanisms of Hematopoietic Stem Cell Expansion}

\subsubsection{Hematopoietic Stem Cell Self-Renewal and the Role of HOXB4}

Self-renewal or expansion of hematopoietic stem and progenitor cells requires the coordinated proliferation signals together with inhibition of apoptosis and differentiation programs.

\subsubsection{Survival Signals}

Inhibition of apoptosis is essential for hematopoietic stem and progenitor cell survival. ${ }^{197 ; 198}$ Quiescent hematopoietic stem cells are resistant to cell cycle checkpoint-induced apoptosis. In stress conditions, such as during in vitro expansion cultures, apoptosis plays a major role in hematopoietic stem and progenitor cell loss. Intrinsic apoptotic and extrinsic apoptotic pathways may both be important for hematopoietic stem cell function.

The intrinsic apoptotic pathway is initiated from an intracellular signal, such as DNA damage, defective cell cycle, loss of cell survival factors, or other types of intracellular stress. This pathway involves the activation of pro-apoptotic Bcl-2 family members, Bax or Bak in the outer mitochondrial membrane, which eventually causes cytochrome $c$ release from the inter-mitochondrial membrane space into cytosol. Cytochrome $c$ in turn binds to the adaptor apoptotic protease activating factor-1, forming the apoptosome, which is able to activate the initiator caspase-9. Capase- 9 can activate the downstream effector capases $-3,-6$ or -7 to cleave a variety of cellular substrates for executing apoptotic response. The anti-apoptotic Bcl-2 proteins, including Bcl-2, Bcl-xL and Mcl-1, act to prevent the mitochondrial outer membrane permeabilization (MOMP) through inhibiting the action of the pro-apoptotic $\mathrm{Bcl}-2$ family proteins. The anti-apoptotic Bcl2 family members are involved in maintaining the function of hematopoietic stem cells. Mice lacking the anti-apoptotic $\mathrm{Bcl}-2$ gene show massive cell death of lymphoid cells. ${ }^{199}$ The absence of Bcl-xL causes embryonic lethality; and homozygous null mutant mice die at E13.0 with massive cell death in neurons and immature hematopoietic cells. ${ }^{200} \mathrm{Bcl}-\mathrm{xL}$ is also indispensable for the late maturation of primitive and definitive erythroid progenitors. ${ }^{200}$ Inducible deletion of $\mathrm{Mcl}-1$ gene in mice results in the loss of early bone marrow progenitor populations including hematopoietic stem cells. ${ }^{70}$ Overexpression of Bcl-2 in HSCs in vivo in a transgenic mouse model results in apoptotic protection, and a moderate two fold expansion of HSCs. ${ }^{22 ; 201}$ Those hematopoietic stem cells overexpressing Bcl-2 
can outcompete wild type cells following reconstitution..$^{22}$ Based on this information, the intrinsic apoptotic pathway is important in hematopoietic stem and progenitor cell expansion.

On the other hand, the extrinsic apoptotic pathway also shows its role in hematopoietic cell expansion. The extrinsic apoptotic pathway starts with an extracellular signal, such as Fas ligand and TNF- $\alpha$, which activates specific death receptors on the cell surface. In this case, the ligand binding induces receptor clustering and recruitment of the adaptor protein Fas-associated death domain (FADD) and the initiator caspases-8 or -10, forming a death-inducing signaling complex (DISC). The initiator caspases then can be processed into their active form and be released into the cytoplasm, where they activate effector caspases $-3,-6$, or -7 . For example, TNF- $\alpha$ is able to inhibit SCF-induced expansion of hematopoietic progenitor cells in vitro. ${ }^{202}$

Our data proves that one mechanism of HOXB4 in promoting hematopoietic stem and progenitor cell self-renewal is through protecting cells from apoptosis by activating its direct downstream target gene-Hemgn. Human HEMGN was shown to act as a transcriptional regulator to upregulate the expression of $\mathrm{Bcl}-2$ and $\mathrm{Bcl}-\mathrm{xL} .{ }^{173}$ Due to their high homology, mouse Hemgn probably has the same ability to regulate the anti-apoptotic $\mathrm{Bcl}-2$ family members to perturb the intrinsic apoptotic pathway. On the other hand, human HEMGN can activate NF-kB, which in turn inhibits TNF- $\alpha$ receptor signaling induced apoptosis. ${ }^{172}$ It is possible that mouse Hemgn can activate NF- $\mathrm{KB}$ to inhibit apoptosis. Intriguingly, HOXB4 was shown to inhibit TNF- $\alpha$ pathway and thereby to protect cells from TNF- $\alpha$ induced apoptosis. ${ }^{144}$ Based on this information, the mechanism of Hemgn in protecting HOXB4-overexpressing cells from apoptosis could be that Hemgn can either inhibit intrinsic apoptotic pathways by upregulating of anti-apoptotic Bcl-2 family members, or inhibit extrinsic apoptotic pathways by interfering with TNF- $\alpha$ receptor signaling. To test these possibilities, examination of both intrinsic and extrinsic apoptotic pathways would be necessary in Hemgn-overexpressed cells. For instance, the expression level of $\mathrm{Bcl}-2, \mathrm{Bcl}-\mathrm{xL}$ and $\mathrm{Mcl}-1$ in Hemgnoverexpressing or -deficient cells could be examined, and inhibition of the NF-kB pathway could be used to discern whether the effects of Hemgn can be overcome.

\subsubsection{Proliferation Signals}

Apoptosis may not be the only mechanism of how HOXB4 functions in promoting hematopoietic stem and progenitor cell self-renewal. Indeed, the fact that HOXB4 can promote 40 fold expansion of hematopoietic stem cells in vitro is not likely to be explained merely by its anti-apoptotic effect. For instance, Bcl-2 overexpression adequately provides a signal for hematopoietic stem cell survival but merely causes a two fold expansion of hematopoietic stem cells because of the inability to maintain those cells in the undifferentiated status. ${ }^{21}$ Other signals, 
presumably those regulating proliferation and differentiation, may also contribute to the dramatic effect mediated by HOXB4.

Cyclins and cyclin-dependent kinases (CDKs) are critical components controlling the cell cycle progression. When activated by a particular cyclin, CDK can activate or inactivate target proteins to orchestrate entry into the next phase of the cell cycle. Upon receiving mitotic signal, $\mathrm{G}_{1}$ cyclin-CDK complexes become active to prepare the cell for $S$ phase entry, through promoting the expression of transcription factors that in turn induce the expression of $S$ cyclins and enzymes required for DNA replication, or through inducing the degradation of factors which function as $S$ phase inhibitors. For example, cyclin A-CDK2 complex plays a key role in $S$ phase entry through the phosphorylation of proteins including cdh1, Rb, p21 and p27. Accelerated S phase entry caused by HOXB4 may significantly contribute to the induced dramatic expansion of hematopoietic stem cells. The lack of alteration in cell cycle distribution in Hemgn-overexpressing bone marrow cells implies the existence of Hemgn-independent proliferative effects of HOXB4. Although it is unknown which other target genes of HOXB4 regulate proliferation, I hypothesize that cell cycle-related genes will be candidates. p21 contributes to the quiescent state maintenance of hematopoietic stem cells. ${ }^{68}$ It has been demonstrated that hematopoietic stem cell pool size is regulated by p21, whereas proliferation of hematopoietic progenitor cells is regulated by p27. ${ }^{203 ; 204}$ Deletion of $p 18$ results in enhanced self-renewal of the primitive hematopoietic cells and improved long-term engraftment. ${ }^{205}$ It is possible that HOXB4 can downregulate the expression of those $\mathrm{CDK}$ inhibitors to facilitate more rapid $\mathrm{S}$ phase entry. Correlatively, when HOXB4 was overexpressed in hematopoietic cells from $p 21^{-/}$mice, the primary CFU-C colony number and the repopulating ability were significantly increased compared to that seen in the wild type bone marrow cells. ${ }^{167}$ These findings suggest that HOXB4 may affect the cell cycle process to promote hematopoietic stem cell self-renewal. However, the fact that we did not detect any differential expression change of cell cycle regulators including cyclins, CDKs and CDK inhibitors in our microarray data suggests that HOXB4 may indirectly regulate the activity of these cell cycle regulators.

\subsubsection{Anti-Differentiation Signals}

Differentiation is the transition process of a less specialized cell into a more specialized cell type. One cycling hematopoietic stem cell may generate two daughter cells with identical biological properties as itself in the process of selfrenewal, or it may also divide to produce more differentiated progenies. During the differentiation, hematopoietic stem cell gradually loses its totipotency, but the generated hematopoietic progenitor cells may still retain a high degree of multipotency.

HOXB4-overexpressed bone marrow cells showed a more homogenous morphology and contained increased number of hematopoietic progenitor cells 
capable of generating primitive colonies in vitro. ${ }^{150}$ In contrast, control-transduced bone marrow cells in long-term cultures showed hallmarks of myeloid differentiation and reduced secondary colony forming activity. ${ }^{150}$ It is reasonable to raise the possibility that HOXB4 has anti-differentiation function. Indeed, evidence shows that ectopic expression of HOXB4 can cause delay or impairment of myeloid differentiation. ${ }^{154 ; 189}$ Similarly, there is also some evidence that Hemgn can interfere with differentiation. Human HEMGN blocks the differentiation of hematopoietic cell lines. ${ }^{172}$ Transgenic expression of human HEMGN under the cd11a promoter in mice causes massive myelopoiesis and repressed lymphlopoiesis. ${ }^{173}$ However, in contrast to these reports, we found that either HOXB4 overexpression or Hemgn overexpression in bone marrow cells did not cause blockage of myeloid differentiation based on the observation of the secondary CFU-C assays in vitro. Furthermore, after the HOXB4 or Hemgn-transduced donor bone marrow cells were transplanted into lethally irradiated recipient mice, peripheral blood from the reconstituted recipient mice were examined to confirm that no hematopoietic lineage was blocked due to the overexpression of HOXB4 or Hemgn. These evidences indicate that HOXB4 or Hemgn may not irreversibly block differentiation of hematopoietic stem and progenitor cells, and thus suggest that HOXB4 or Hemgn could be good candidates for therapeutic application since they are not potentially tumorigenic.

\subsubsection{Cytokines, HOXB4 and Hemgn}

It is known that in hematopoietic tissues, Hoxb4 expression can be upregulated by cytokine-stimulation. TPO, Flt-3 or SCF were reported to induce Hoxb4 expression up to 10 fold through activating its direct trimeric transcriptional activator complex USF1 (upstream stimulating factor 1)/USF2/NF$\mathrm{Y}$ (nuclear factor $\mathrm{Y}$ ). ${ }^{206-208}$ Cytokine receptor signals, such as TPOR, FIt3R and c$\mathrm{KIT}$, are normally required for hematopoietic stem cell survival during in vitro expansion. Now we provide potentially new insights into the downstream pathways of these cytokines and HOXB4 through identification of a key regulator-Hemgn. It is possible that Hemgn can be directly induced by Hoxb4 following cytokine stimulation and thus provide a signal for hematopoietic stem and progenitor cell survival. Human HEMGN was shown to act as a transcriptional regulator to upregulate the expression of $\mathrm{Bcl}-2$ and $\mathrm{Bcl}-\mathrm{xL} .{ }^{173}$ Due to high homology, mouse Hemgn probably has the same ability to regulate the anti-apoptotic Bcl-2 family members to perturb the intrinsic apoptotic pathway. The fact that hematopoietic stem cells from Bcl-2 transgenic mice are hyperresponsive to normal level cytokines than wild type cells ${ }^{21}$ is correlated to the hypothesis that cytokine signaling may upregulate Hemgn which may further upregulate $\mathrm{Bcl}-2$. Consistently, we have shown that Hemgn was able to protect bone marrow cells from cytokine withdrawal-induced apoptosis. This suggests that Hemgn might substitute for the function of those cytokines to support cell survival. To test this possibility, we can compare cultures with or without 
hematopoietic cytokines IL-3, IL-6 and SCF to see whether Hemgn can be induced by these cytokines.

\subsubsection{HOXB4 Functional Redundancy}

Hoxb4 and its other three paralogs: Hoxa4, Hoxc4 and Hoxd4 were reported to have similar effect in promoting hematopoietic stem and progenitor cell expansion in vitro. ${ }^{167}$ Each paralog group 4 member was shown to both promote proliferation and inhibit differentiation, enabling the exponential expansion of hematopoietic progenitors from the c-kit ${ }^{+} \mathrm{CD} 41^{+}$hematopoietic progenitor cell population of day 6 murine embryoid bodies. ${ }^{120}$ Similar findings about the functions of HOX4 paralogs in promoting hematopoietic stem and progenitor cell expansion in vivo were also obtained in our lab (Brian P Sorrentino's lab, unpublished data). This redundancy may explain the mild phenotype in $\mathrm{Hoxb}^{-/-}$ mice. ${ }^{158}$ Quantitative analysis of mRNA from the fetal liver of $\mathrm{Hoxb4}{ }^{-/-}$mice revealed that a deficiency of Hoxb4 was associated with changes in the expression levels of several other Hox genes. ${ }^{158}$ This result indicates that alternative genetic pathways initiated by other Hox4 paralog members could substitute for the function of Hoxb4. Since trans-paralog Hox genes share higher degree of homology in sequence than cis-paralog Hox genes, ${ }^{104}$ it is suggested that the same trans-paralog genes could functionally compensate for each other. As Hox4 paralog genes lie on different chromosomes, it is feasible to examine the degree of redundancy among them by breeding the mice with individual disrupted gene. Although technically challenging, the analysis of double, triple and even quadruple mutants of Hox4 paralogy genes could address the question whether these Hox4 genes are functionally redundant in regulating hematopoiesis.

Overexpression of HOXB4 in ES cell-derived primitive progenitors induced a switch to the definitive hematopoietic stem cell phenotype and promoted rapid hematopoietic stem cell expansion in vitro. ${ }^{146}$ Those HOXB4 induced progenitors from ES cells engraft lethally irradiated adults and contribute to long-term, multilineage hematopoiesis in primary and secondary recipients. ${ }^{146}$ During embryogenesis, primitive hematopoiesis occurs in blood islands of the yolk sac at approximately E7.5, and generates mainly primitive erythrocytes. The definitive hematopoiesis occurs around E10.5 in aorta-gonadmesonephros (AGM) regions, and then shifts to the fetal liver, and eventually to the spleen and bone marrow in adult. ${ }^{209}$ The definitive hematopoiesis generates all hematopoietic lineages, including hematopoietic stem cells with multipotent longterm repopulating activity. The mechanisms regulating the emergence of the definitive hematopoietic stem cells at AGM region around E10.5 and the further rapid expansion is poorly understood. Evidence shows that the autonomous initiation and further expansion of definitive hematopoietic stem cells in the AGM region can be enhanced by application of exogenous interleukin-3 (IL-3) which acts as a proliferation and survival factor to the earliest hematopoietic stem cells 
in the embryo. ${ }^{210}$ The identified expression pattern of Hoxb4 and its other Hox4 paralogy members in definitive hematopoietic stem cells but not yolk sac suggests the potential role of Hoxb4 and other Hox4 paralogy members in promoting definitive hematopoiesis. ${ }^{105}$ Since Hoxb4 can be regulated by some hematopoietic cytokines, it is possible that Hoxb4 acts downstream of IL-3 to convey its function in enhancing the development of definitive hematopoietic stem cells in embryo. Although the Hoxb4-dificient mice show essentially normal hematopoiesis, deletion of the other three functionally redundant Hox4 paralogy members could possibly lead to more severe phenotype in embryonic hematopoietic stem cell development.

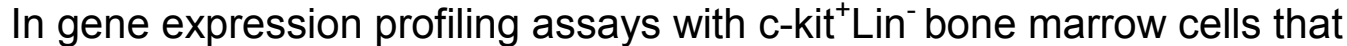
constitutively overexpress HOXB4 or HOXC4 by retroviral vectors, we found that many genes, including Hemgn, were commonly changed in HOXB4 and in HOXC4 overexpressed samples compared to the control vector-transduced samples (Brian P. Sorrentino's lab, unpublished data). It is possible that HOX4 paralogs share at least some common downstream targets, such as Hemgn, for enhancing expansion of hematopoietic stem and progenitor cells. Our real-time PCR analysis in Hoxb4 ${ }^{-/}$c-kit ${ }^{+}$Scal- $1^{+}$Lin $^{-}$cells showed a 5 -fold decrease in Hemgn mRNA expression when compared to wild type cells. This result is not contradictory to the finding that Hemgn is a direct target of HOXB4 for conveying its function in promoting self-renewal, because the expression of Hemgn can still be detected in the absence of Hoxb4 possibly due to redundancy of other group 4 paralogs. Investigation of whether Hemgn is a common direct target gene of HOX4 paralog members is promising to unravel the mechanisms of HOX4 paralogs.

\subsubsection{HOX Specificity}

Functional specificity of Hox proteins depends on multiple mechanisms. Due to the high degree of homology within the homeodomain, a core DNA sequence "TAAT" is recognized and bound by the majority of Hox proteins. ${ }^{128}$ Structural studies proved that DNA recognition by Hox proteins uses two sets of information that provide different degrees of specificity. The first one uses DNA-contacting residues that are common to all Hox proteins to promote Hox binding to AT-rich sequences, such as "TAAT". Some monomeric homeodomain-DNA structures have been solved, and they show a very similar mode of DNA recognition. ${ }^{211}$ The third recognition a-helix of homeodomain lies in the major groove of the DNA, where it makes direct water-mediated contacts with specific bases and the phosphate backbone. Ile47, GIn50, Asn51, and Met54 residues that are present in all Hox homeodomains are primarily responsible for contributing to these interactions. ${ }^{211}$ In addition, the $\mathrm{N}$-terminal arm preceding the first $\alpha$-helix typically lies in the minor groove of the DNA. Arg5, an N-terminal arm residue present in almost all homeodomains, is the most commonly observed residue in the minor groove. The second set of determination factor for specificity in addition to 
common DNA-contacting residues is to use additional DNA-contacting residues from the $\mathrm{N}$-terminal arm and linker regions of Hox proteins. ${ }^{212}$ These additional contacts are cofactor-dependent and paralog-specific. The N-terminal arm and linker residues of Hox family members are conserved in a paralog-specific manner. Thus, paralog-specific DNA sequences of Hox family members could be recognized of by specific Hox-cofactor complexes. The same Hox paralogy members probably share common targets for functional complement to initiate the similar downstream biological events.

As discussed previously, the DNA binding specificity, affinity and transregulating potential of a specific Hox protein can be modified by the flanking DNA sequence or interaction with cofactors. ${ }^{129-131} \mathrm{~A}$ in vitro study showed that specific Hox protein is able to bind to DNA consensus sequence with preferred flanking sequence. ${ }^{129}$ In the future, by applying ChIP-chip and ChIP-seq methodologies to each HOX protein, accurate DNA binding sequence of specific HOX protein could be identified. Hox family members may bind to their specific preferred DNA sequence as monomers or as heterodimers with other cofactors. However, when high affinity Hox monomer binding sites were applied in vitro to drive a reporter gene in Drosophila embryos, the generated expression patterns were not consistent with their identified ability to bind homeodomain proteins. ${ }^{213}$ It is plausible to hypothesize that currently unidentified factors can bind with Hox proteins cooperatively or noncooperatively to these sites. As introduced in Chapter 1, interaction with the TALE family cofactors to form dimeric or trimeric Hox-Pbx/Meis complexes is hypothesized to increase the DNA binding ability of some Hox family members to their target genes. ${ }^{136-138}$

DNA binding affinity of Hox family members can also be regulated by posttranslational modification. For instance, phosphorylation of conserved tyrosine residues in the homeodomain can decrease or increase the DNA binding ability of Hox. ${ }^{133 ; 134}$ In addition, the transcriptional specificity of Hox proteins also lies in their ability to act both as transcriptional activators and repressors in different contexts.

The fact that HOXB4 and other HOX4 paralog members but not other HOX family member show similar function in promoting hematopoietic stem and progenitor cell expansion indicates the specificity of HOX4 in regulating their target genes in hematopoietic cells. To my understanding, although it is known that PBX cofactor is not required for HOXB4-mediated expansion, it is possible that other unidentified cofactors or collaborators of HOXB4 exist. To screen the potential cofactor or collaborators of HOXB4, one could apply proteomics methods following immunoprecipitation of HOXB4-containing complex. After that, one could knockdown the identified interaction partner in HOXB4-overexpressed cells to validate its function. Except the conserved "YPWM" cofactor-binding domain, mutagenesis screen method could be applied in HOXB4 to identify other unknown protein-interaction domains which contribute to its specificity. On the other hand, phosphorylation could possibly increase the target binding affinity of 
HOXB4. HOXB4 was tyrosine phosphorylated in the transduced mouse and dog cells, in which HOXB4 was introduced with high copy transcripts and thus high protein expression level. ${ }^{214}$ Furthermore, the tyrosine phosphorylation of HOXB4 contributes to more profound expansion in the transduced cells. ${ }^{214}$ Whether HOXB4 is phosphorylated in the transduced bone marrow cells can be determined by mass spectrometry following immunoprecipitation of HOXB4. If HOXB4 is phosphorylated, one could make point mutation (Ser/Thr/Tyr/ to Ala) in phosphorylation sites and determine whether this modification abrogates the ability of HOXB4 to activate target gene expression and thereby blunts its ability to increase self-renewal.

\subsubsection{HOXB4 and Leukemia}

Hematopoietic stem cells may fail to fully regenerate the stem cell compartment since their self-renewal ability is usually intrinsically limited and subjected to exhaustion under the transplantation stress. This defect is possibly a result of negative feedback mechanisms initiated when progenitors and mature blood cells have been regenerated to their normal levels, which prematurely inhibit further hematopoietic stem cell expansion. ${ }^{215 ; 216}$ In contrast, HOXB4transduced hematopoietic stem cells are capable of fully reconstituting the stem cell compartment but do not expand above levels normally observed in unmanipulated mice. ${ }^{89}$ This indicates that HOXB4 overexpression does not override the intrinsic regulatory mechanisms in vivo that maintain the HSC pool size within normal limits. Several studies have indicated that, in steady-state hematopoiesis, the cell cycle entry of hematopoietic stem cells is tightly controlled. In mice, hematopoietic stem cell numbers remain relatively constant throughout their adult life time. Hematopoietic stem cells may transit between quiescent state in niche and undergoing cycling at non-niche sites in vivo. Genetic studies show that increasing the number of osteoblastic cells can cause parallel increases in the hematopoietic stem cell population, in contrast, osteoblastic cell depletion can reduce BM cellularity. ${ }^{217 ; 218}$ The finding that HOXB4 overexpression did not lead to hematopoietic stem cell expansion beyond the normal level is thus not surprising, since the limited size of hematopoietic stem cell niche could prevent homing and residence of more hematopoietic stem cells.

Despite a profound and consistent effect on expanding hematopoietic stem and progenitor cells, overexpression of HOXB4 did not promote preferential expansion along any hematopoietic lineage or lead to leukemia in mice after persistent expression. In sharp contrast, retroviral overexpression of other HOX family members such as HOXB3 or HOXA10 in a similar transplantation model did lead to leukemia. ${ }^{118 ; 219}$ The different outcomes suggest that these different HOX proteins regulate different sets of target genes in hematopoietic cells. A minimum of two events, one leading to enhanced proliferation with disabled apoptosis and the other a block in differentiation, may be sufficient for leukemia initiation. ${ }^{220 ; 221}$ Of the known genetic events leading to leukemia, translocations 
leading to fusion transcription factors or activated signaling kinases are among the most common events, and deletions in cell cycle checkpoint genes or mutation in tumor suppressor genes could act as a secondary hit to initiate leukemic transformation. ${ }^{222}$ One possible reason HOXB4 overexpression did not cause leukemia in mice is that the intrinsic negative feedback regulatory signaling pathways were activated to limit the accumulation of leukemic transformation hits.

In contrast to the situation in mouse models as we discussed, myeloid leukemia was reported in dogs two years after they were transplanted with HOXB4-overexpressing CD $34^{+}$cells, but not in control retroviral vector transplanted animals. ${ }^{157}$ shRNA-mediated knockdown of HOXB4 in the leukemic cell line derived from one animal caused profound growth inhibition and cell death, suggesting the involvement of HOXB4 in leukemogenesis. ${ }^{157}$ Those HOXB4-overexpressing leukemic cells rapidly engrafted and expanded in immune-suppressed recipient dogs to cause leukemia transmission. ${ }^{223}$ This, although surprising, is not contradictory to the previous findings in mouse models, where HOXB4 is not leukemogenic. Instead, in this case, HOXB4 overexpression was demonstrated not to be the only contributor to the observed leukemic phenotype in dogs. ${ }^{157 ; 224}$ Retroviruses have long been known to cause leukemia through insertional mutagenesis. In those two transplanted dogs, HOXB4containing retroviral vectors were shown to integrate into genomic locus of several known proto-oncogenes, including c-myb and PRDM16, to elevate their expression, thus favoring the engraftment and expansion of those clones. ${ }^{157 ; 224}$ HOXB4 overexpression is thus probably not the single trigger to induce leukemia; instead, it may function as an additional contributor for leukemogenesis following retroviral vector integration-caused insertional mutagenesis. One reason that HOXB4-overexpression leads to leukemia in dogs but not in mice possibly lies in the fact that a relatively small number of transduced cells and relatively less retroviral vector integration sites in transplanted mice lower the frequency of insertional mutagenesis. ${ }^{157 ; 224}$ On the other hand, perhaps a latency period longer than the lifespan of a mouse is required for detection of leukemic transformation. ${ }^{157 ; 224}$ In summary, the observed myeloid leukemia is probably a combined consequence of the deregulated proliferation caused by the activated proto-oncogenes and the expansion advantage mediated by HOXB4.$^{157 ; 224}$

Based on the information aforementioned, one could speculate that as a potential application in gene therapy, retrovirus-mediated overexpression of HOXB4 in large animals may pose a significant risk of leukemia. To circumvent this drawback, new vectors based on lentivirus are in development, which may decrease the frequency of insertional mutagenesis. ${ }^{158}$ 


\section{LIST OF REFERENCES}

1. Smith $\mathrm{C}$. Hematopoietic stem cells and hematopoiesis. Cancer Control 2003;10:9-16.

2. Weissman IL. Stem cells: units of development, units of regeneration, and units in evolution. Cell 2000;100:157-168.

3. Weissman IL, Anderson DJ, Gage F. Stem and progenitor cells: origins, phenotypes, lineage commitments, and transdifferentiations. Annu.Rev.Cell Dev.Biol. 2001;17:387-403.

4. Lemischka I. Stem cell dogmas in the genomics era. Rev.Clin.Exp.Hematol. 2001;5:15-25.

5. Eaves CJ, Eaves AC. Stem cell kinetics. Baillieres Clin.Haematol. 1997;10:233-257.

6. Eaves C, Miller C, Cashman J et al. Hematopoietic stem cells: inferences from in vivo assays. Stem Cells 1997;15 Suppl 1:1-5.

7. Domen J. The role of apoptosis in regulating hematopoietic stem cell numbers. Apoptosis 2001;6:239-252.

8. Domen J. The role of apoptosis in regulating hematopoiesis and hematopoietic stem cells. Immunol.Res. 2000;22:83-94.

9. Domen J, Weissman IL. Self-renewal, differentiation or death: regulation and manipulation of hematopoietic stem cell fate. Mol.Med.Today 1999;5:201-208.

10. Domen J, Gandy KL, Weissman IL. Systemic overexpression of BCL-2 in the hematopoietic system protects transgenic mice from the consequences of lethal irradiation. Blood 1998;91:2272-2282.

11. Till JE, Mcculloch EA. A direct measurement of the radiation sensitivity of normal mouse bone marrow cells. Radiat.Res. 1961;14:213-222.

12. Orkin SH. Diversification of haematopoietic stem cells to specific lineages. Nat.Rev.Genet. 2000;1:57-64.

13. Lansdorp PM, Schmitt C, Sutherland HJ et al. Hemopoietic stem cell characterization. Prog.Clin.Biol.Res. 1992;377:475-484.

14. Palis J, Yoder MC. Yolk-sac hematopoiesis: the first blood cells of mouse and man. Exp.Hematol. 2001;29:927-936. 
15. Dzierzak E, Medvinsky A, Bruijn M. Qualitative and quantitative aspects of haematopoietic cell development in the mammalian embryo.

Immunol.Today 1998;19:228-236.

16. Osawa M, Hanada K, Hamada H, Nakauchi H. Long-term lymphohematopoietic reconstitution by a single CD34-low/negative hematopoietic stem cell. Science 1996;273:242-245.

17. Chen J, Ellison FM, Keyvanfar $\mathrm{K}$ et al. Enrichment of hematopoietic stem cells with SLAM and LSK markers for the detection of hematopoietic stem cell function in normal and Trp53 null mice. Exp.Hematol. 2008;36:12361243.

18. Uchida N, Weissman IL. Searching for hematopoietic stem cells: evidence that Thy-1.1lo Lin- Sca-1+ cells are the only stem cells in C57BL/Ka-Thy1.1 bone marrow. J.Exp.Med. 1992;175:175-184.

19. Morrison SJ, Weissman IL. The long-term repopulating subset of hematopoietic stem cells is deterministic and isolatable by phenotype. Immunity 1994;1:661-673.

20. Attar EC, Scadden DT. Regulation of hematopoietic stem cell growth. Leukemia 2004;18:1760-1768.

21. Domen J, Weissman IL. Hematopoietic stem cells need two signals to prevent apoptosis; BCL-2 can provide one of these, Kitl/c-Kit signaling the other. J.Exp.Med. 2000;192:1707-1718.

22. Domen J, Cheshier SH, Weissman IL. The role of apoptosis in the regulation of hematopoietic stem cells: overexpression of $\mathrm{Bcl}-2$ increases both their number and repopulation potential. J.Exp.Med. 2000;191:253264.

23. Scadden DT. The stem-cell niche as an entity of action. Nature 2006;441:1075-1079.

24. Frisch BJ, Porter RL, Calvi LM. Hematopoietic niche and bone meet. Curr.Opin.Support.Palliat.Care 2008;2:211-217.

25. Magnusson M, Mikkola HK. Hematopoietic stem cells in transit--where's the niche? Cell Stem Cell 2008;2:302-304.

26. Martinez-Agosto JA, Mikkola HK, Hartenstein V, Banerjee U. The hematopoietic stem cell and its niche: a comparative view. Genes Dev. 2007;21:3044-3060.

27. Li Z, Li L. Understanding hematopoietic stem-cell microenvironments. Trends Biochem.Sci. 2006;31:589-595. 
28. Haylock DN, Nilsson SK. Stem cell regulation by the hematopoietic stem cell niche. Cell Cycle 2005;4:1353-1355.

29. Hackney JA, Charbord P, Brunk BP et al. A molecular profile of a hematopoietic stem cell niche. Proc.Natl.Acad.Sci.U.S.A 2002;99:1306113066.

30. Moore KA, Lemischka IR. Stem cells and their niches. Science 2006;311:1880-1885.

31. Akala OO, Clarke MF. Hematopoietic stem cell self-renewal. Curr.Opin.Genet.Dev. 2006;16:496-501.

32. Zon LI. Intrinsic and extrinsic control of haematopoietic stem-cell selfrenewal. Nature 2008;453:306-313.

33. Lo CC, Klein RJ, Scadden DT. Analysis of the hematopoietic stem cell niche. Curr.Protoc.Stem Cell Biol. 2007;3:1-31.

34. Magnusson M, Mikkola HK. Hematopoietic stem cells in transit--where's the niche? Cell Stem Cell 2008;2:302-304.

35. Calvi LM, Adams GB, Weibrecht KW et al. Osteoblastic cells regulate the haematopoietic stem cell niche. Nature 2003;425:841-846.

36. Zhang J, Niu C, Ye L et al. Identification of the haematopoietic stem cell niche and control of the niche size. Nature 2003;425:836-841.

37. Yokota $\mathrm{T}$, Oritani K, Mitsui $\mathrm{H}$ et al. Growth-supporting activities of fibronectin on hematopoietic stem/progenitor cells in vitro and in vivo: structural requirement for fibronectin activities of CS1 and cell-binding domains. Blood 1998;91:3263-3272.

38. Arai $F$, Hirao A, Ohmura $M$ et al. Tie2/angiopoietin-1 signaling regulates hematopoietic stem cell quiescence in the bone marrow niche. Cell 2004;118:149-161.

39. Takakura N, Huang XL, Naruse T et al. Critical role of the TIE2 endothelial cell receptor in the development of definitive hematopoiesis. Immunity 1998;9:677-686.

40. Karanu FN, Murdoch B, Gallacher L et al. The notch ligand jagged-1 represents a novel growth factor of human hematopoietic stem cells. J.Exp.Med. 2000;192:1365-1372.

41. Karanu FN, Murdoch B, Miyabayashi T et al. Human homologues of Delta1 and Delta-4 function as mitogenic regulators of primitive human hematopoietic cells. Blood 2001;97:1960-1967. 
42. Ohishi K, Varnum-Finney B, Bernstein ID. Delta-1 enhances marrow and thymus repopulating ability of human CD34(+)CD38(-) cord blood cells. J.Clin.Invest. 2002;110:1165-1174.

43. Varnum-Finney B, Purton LE, Yu M et al. The Notch ligand, Jagged-1, influences the development of primitive hematopoietic precursor cells. Blood 1998;91:4084-4091.

44. Varnum-Finney B, Xu L, Brashem-Stein C et al. Pluripotent, cytokinedependent, hematopoietic stem cells are immortalized by constitutive Notch1 signaling. Nat.Med. 2000;6:1278-1281.

45. Duncan AW, Rattis FM, DiMascio LN et al. Integration of Notch and Wnt signaling in hematopoietic stem cell maintenance. Nat.Immunol. 2005;6:314-322.

46. Stier S, Cheng T, Dombkowski D, Carlesso N, Scadden DT. Notch1 activation increases hematopoietic stem cell self-renewal in vivo and favors lymphoid over myeloid lineage outcome. Blood 2002;99:2369-2378.

47. Ye Q, Shieh JH, Morrone G, Moore MA. Expression of constitutively active Notch4 (Int-3) modulates myeloid proliferation and differentiation and promotes expansion of hematopoietic progenitors. Leukemia 2004;18:777787.

48. Willert K, Brown JD, Danenberg E et al. Wnt proteins are lipid-modified and can act as stem cell growth factors. Nature 2003;423:448-452.

49. Reya $T$, Duncan AW, Ailles $L$ et al. A role for $W n t$ signalling in self-renewal of haematopoietic stem cells. Nature 2003;423:409-414.

50. Rane SG, Reddy EP. JAKs, STATs and Src kinases in hematopoiesis. Oncogene 2002;21:3334-3358.

51. Heike T, Nakahata T. In vitro expansion of hematopoietic stem cells by cytokines. Biochim.Biophys.Acta 2002;1592:313-321.

52. Nakauchi $\mathrm{H}$, Sudo K, Ema H. Quantitative assessment of the stem cell selfrenewal capacity. Ann.N.Y.Acad.Sci. 2001;938:18-24.

53. Petzer AL, Zandstra PW, Piret JM, Eaves CJ. Differential cytokine effects on primitive (CD34+CD38-) human hematopoietic cells: novel responses to Flt3-ligand and thrombopoietin. J.Exp.Med. 1996;183:2551-2558.

54. Sitnicka E, Lin N, Priestley GV et al. The effect of thrombopoietin on the proliferation and differentiation of murine hematopoietic stem cells. Blood 1996;87:4998-5005. 
55. Ueda T, Tsuji K, Yoshino $\mathrm{H}$ et al. Expansion of human NOD/SCIDrepopulating cells by stem cell factor, Flk2/FIt3 ligand, thrombopoietin, IL-6, and soluble IL-6 receptor. J.Clin.Invest. 2000;105:1013-1021.

56. Luskey BD, Rosenblatt M, Zsebo K, Williams DA. Stem cell factor, interleukin-3, and interleukin-6 promote retroviral-mediated gene transfer into murine hematopoietic stem cells. Blood 1992;80:396-402.

57. Cassel A, Cottler-Fox M, Doren S, Dunbar CE. Retroviral-mediated gene transfer into CD34-enriched human peripheral blood stem cells.

Exp.Hematol. 1993;21:585-591.

58. Crooks GM, Hao QL, Petersen D, Barsky LW, Bockstoce D. IL-3 increases production of $B$ lymphoid progenitors from human CD34+CD38- cells. J.Immunol. 2000;165:2382-2389.

59. Haylock DN, Horsfall MJ, Dowse TL et al. Increased recruitment of hematopoietic progenitor cells underlies the in vitro expansion potential of FLT3 ligand. Blood 1997;90:2260-2272.

60. Lyman SD, Jacobsen SE. C-kit ligand and FIt3 ligand: stem/progenitor cell factors with overlapping yet distinct activities. Blood 1998;91:1101-1134.

61. Massague $\mathrm{J}$. The transforming growth factor-beta family. Annu.Rev.Cell Biol. 1990;6:597-641.

62. Roberts AB, Anzano MA, Wakefield LM et al. Type beta transforming growth factor: a bifunctional regulator of cellular growth.

Proc.Natl.Acad.Sci.U.S.A 1985;82:119-123.

63. Cardoso AA, Li ML, Batard P et al. Release from quiescence of CD34+. Proc.Natl.Acad.Sci.U.S.A 1993;90:8707-8711.

64. Li ML, Cardoso AA, Sansilvestri P et al. Additive effects of steel factor and antisense TGF-beta 1 oligodeoxynucleotide on CD34+ hematopoietic progenitor cells. Leukemia 1994;8:441-445.

65. Bhardwaj G, Murdoch B, Wu D et al. Sonic hedgehog induces the proliferation of primitive human hematopoietic cells via BMP regulation. Nat.Immunol. 2001;2:172-180.

66. Bhatia M, Bonnet $\mathrm{D}, \mathrm{Wu} \mathrm{D}$ et al. Bone morphogenetic proteins regulate the developmental program of human hematopoietic stem cells. J.Exp.Med. 1999;189:1139-1148.

67. Cheng T, Rodrigues N, Dombkowski D, Stier S, Scadden DT. Stem cell repopulation efficiency but not pool size is governed by p27(kip1). Nat.Med. 2000;6:1235-1240. 
68. Cheng T, Rodrigues N, Shen $\mathrm{H}$ et al. Hematopoietic stem cell quiescence maintained by p21cip1/waf1. Science 2000;287:1804-1808.

69. Yuan Y, Shen H, Franklin DS, Scadden DT, Cheng T. In vivo self-renewing divisions of haematopoietic stem cells are increased in the absence of the early G1-phase inhibitor, p18INK4C. Nat.Cell Biol. 2004;6:436-442.

70. Opferman JT, Iwasaki H, Ong CC et al. Obligate role of anti-apoptotic MCL1 in the survival of hematopoietic stem cells. Science 2005;307:1101-1104.

71. Yilmaz $\mathrm{OH}$, Valdez $\mathrm{R}$, Theisen $\mathrm{BK}$ et al. Pten dependence distinguishes haematopoietic stem cells from leukaemia-initiating cells. Nature 2006;441:475-482.

72. Zhang J, Grindley JC, Yin T et al. PTEN maintains haematopoietic stem cells and acts in lineage choice and leukaemia prevention. Nature 2006;441:518-522.

73. Melotti P, Calabretta B. The transcription factors c-myb and GATA-2 act independently in the regulation of normal hematopoiesis.

Proc.Natl.Acad.Sci.U.S.A 1996;93:5313-5318.

74. Schmidt M, Nazarov V, Stevens L, Watson R, Wolff L. Regulation of the resident chromosomal copy of c-myc by c-Myb is involved in myeloid leukemogenesis. Mol.Cell Biol. 2000;20:1970-1981.

75. Mucenski ML, McLain K, Kier AB et al. A functional c-myb gene is required for normal murine fetal hepatic hematopoiesis. Cell 1991;65:677-689.

76. Takihara $\mathrm{Y}$, Tomotsune D, Shirai $\mathrm{M}$ et al. Targeted disruption of the mouse homologue of the Drosophila polyhomeotic gene leads to altered anteroposterior patterning and neural crest defects. Development 1997;124:3673-3682.

77. Park IK, Qian D, Kiel M et al. Bmi-1 is required for maintenance of adult self-renewing haematopoietic stem cells. Nature 2003;423:302-305.

78. Lessard J, Sauvageau G. Bmi-1 determines the proliferative capacity of normal and leukaemic stem cells. Nature 2003;423:255-260.

79. Molofsky AV, Pardal R, Iwashita T et al. Bmi-1 dependence distinguishes neural stem cell self-renewal from progenitor proliferation. Nature 2003;425:962-967.

80. Iwama A, Oguro H, Negishi $\mathrm{M}$ et al. Enhanced self-renewal of hematopoietic stem cells mediated by the polycomb gene product Bmi-1. Immunity 2004;21:843-851. 
81. Ohta H, Sawada A, Kim JY et al. Polycomb group gene rae28 is required for sustaining activity of hematopoietic stem cells. J.Exp.Med. 2002;195:759-770.

82. Kim JY, Sawada A, Tokimasa S et al. Defective long-term repopulating ability in hematopoietic stem cells lacking the Polycomb-group gene rae28. Eur.J.Haematol. 2004;73:75-84.

83. Kajiume T, Ninomiya $\mathrm{Y}$, Ishihara H, Kanno R, Kanno M. Polycomb group gene mel-18 modulates the self-renewal activity and cell cycle status of hematopoietic stem cells. Exp.Hematol. 2004;32:571-578.

84. Zeng H, Yucel R, Kosan C, Klein-Hitpass L, Moroy T. Transcription factor Gfi1 regulates self-renewal and engraftment of hematopoietic stem cells. EMBO J. 2004;23:4116-4125.

85. Hock H, Hamblen MJ, Rooke $\mathrm{HM}$ et al. Gfi-1 restricts proliferation and preserves functional integrity of haematopoietic stem cells. Nature 2004;431:1002-1007.

86. Kato $\mathrm{Y}$, Iwama A, Tadokoro $\mathrm{Y}$ et al. Selective activation of STAT5 unveils its role in stem cell self-renewal in normal and leukemic hematopoiesis. J.Exp.Med. 2005;202:169-179.

87. Snow JW, Abraham N, Ma MC et al. STAT5 promotes multilineage hematolymphoid development in vivo through effects on early hematopoietic progenitor cells. Blood 2002;99:95-101.

88. Chung YJ, Park BB, Kang YJ et al. Unique effects of Stat3 on the early phase of hematopoietic stem cell regeneration. Blood 2006;108:1208-1215.

89. Antonchuk J, Sauvageau G, Humphries RK. HOXB4 overexpression mediates very rapid stem cell regeneration and competitive hematopoietic repopulation. Exp.Hematol. 2001;29:1125-1134.

90. Antonchuk J, Sauvageau G, Humphries RK. HOXB4-induced expansion of adult hematopoietic stem cells in vitro. Cell 2002;109:39-45.

91. Sorrentino BP. Clinical strategies for expansion of haematopoietic stem cells. Nat.Rev.Immunol. 2004;4:878-888.

92. Hofmeister CC, Zhang J, Knight KL, Le P, Stiff PJ. In vitro expansion of umbilical cord blood stem cells for transplantation: growing knowledge from the hematopoietic niche. Bone Marrow Transplant. 2007;39:11-23.

93. Eaves C, Miller C, Cashman J et al. Hematopoietic stem cells: inferences from in vivo assays. Stem Cells 1997;15 Suppl 1:1-5. 
94. Ema $\mathrm{H}$, Takano $\mathrm{H}$, Sudo $\mathrm{K}$, Nakauchi $\mathrm{H}$. In vitro self-renewal division of hematopoietic stem cells. J.Exp.Med. 2000;192:1281-1288.

95. Sauvageau G, Thorsteinsdottir U, Eaves CJ et al. Overexpression of HOXB4 in hematopoietic cells causes the selective expansion of more primitive populations in vitro and in vivo. Genes Dev. 1995;9:1753-1765.

96. Kyba M, Perlingeiro RC, Daley GQ. HoxB4 confers definitive lymphoidmyeloid engraftment potential on embryonic stem cell and yolk sac hematopoietic progenitors. Cell 2002;109:29-37.

97. McKinney-Freeman SL, Lengerke C, Jang IH et al. Modulation of murine embryonic stem cell-derived CD41+c-kit+ hematopoietic progenitors by ectopic expression of Cdx genes. Blood 2008;111:4944-4953.

98. Daley GQ. Prospects for stem cell therapeutics: myths and medicines. Curr.Opin.Genet.Dev. 2002;12:607-613.

99. Takahashi K, Yamanaka S. Induction of pluripotent stem cells from mouse embryonic and adult fibroblast cultures by defined factors. Cell 2006;126:663-676.

100. Duboule D, Dolle $P$. The structural and functional organization of the murine HOX gene family resembles that of Drosophila homeotic genes. EMBO J. 1989;8:1497-1505.

101. Levine M, Rubin GM, Tjian R. Human DNA sequences homologous to a protein coding region conserved between homeotic genes of Drosophila. Cell 1984;38:667-673.

102. Akam M. Hox and HOM: homologous gene clusters in insects and vertebrates. Cell 1989;57:347-349.

103. Mark M, Rijli FM, Chambon P. Homeobox genes in embryogenesis and pathogenesis. Pediatr.Res. 1997;42:421-429.

104. Scott MP. Vertebrate homeobox gene nomenclature. Cell 1992;71:551-553.

105. Sauvageau G, Lansdorp PM, Eaves CJ et al. Differential expression of homeobox genes in functionally distinct CD34+ subpopulations of human bone marrow cells. Proc.Natl.Acad.Sci.U.S.A 1994;91:12223-12227.

106. Gehring WJ, Affolter M, Burglin T. Homeodomain proteins. Annu.Rev.Biochem. 1994;63:487-526.

107. Manak JR, Scott MP. A class act: conservation of homeodomain protein functions. Dev.Suppl. 1994;61-77. 
108. Fujino T, Yamazaki Y, Largaespada DA et al. Inhibition of myeloid differentiation by Hoxa9, Hoxb8, and Meis homeobox genes. Exp.Hematol. 2001;29:856-863.

109. Drabkin HA, Parsy C, Ferguson $\mathrm{K}$ et al. Quantitative HOX expression in chromosomally defined subsets of acute myelogenous leukemia. Leukemia 2002;16:186-195.

110. Giampaolo A, Felli N, Diverio D et al. Expression pattern of HOXB6 homeobox gene in myelomonocytic differentiation and acute myeloid leukemia. Leukemia 2002;16:1293-1301.

111. Golub TR, Slonim DK, Tamayo P et al. Molecular classification of cancer: class discovery and class prediction by gene expression monitoring. Science 1999;286:531-537.

112. Lawrence HJ, Sauvageau G, Ahmadi $\mathrm{N}$ et al. Stage- and lineage-specific expression of the HOXA10 homeobox gene in normal and leukemic hematopoietic cells. Exp.Hematol. 1995;23:1160-1166.

113. Kappen C. Disruption of the homeobox gene Hoxb-6 in mice results in increased numbers of early erythrocyte progenitors. Am.J.Hematol. 2000;65:111-118.

114. Lawrence HJ, Helgason CD, Sauvageau G et al. Mice bearing a targeted interruption of the homeobox gene HOXA9 have defects in myeloid, erythroid, and lymphoid hematopoiesis. Blood 1997;89:1922-1930.

115. Slape C, Lin YW, Hartung $\mathrm{H}$ et al. NUP98-HOX translocations lead to myelodysplastic syndrome in mice and men. J.Natl.Cancer Inst.Monogr. 2008;39:64-68.

116. Tosic N, Stojiljkovic M, Colovic N, Colovic M, Pavlovic S. Acute myeloid leukemia with NUP98-HOXC13 fusion and FLT3 internal tandem duplication mutation: case report and literature review. Cancer Genet.Cytogenet. 2009;193:98-103.

117. Slape C, Liu LY, Beachy S, Aplan PD. Leukemic transformation in mice expressing a NUP98-HOXD13 transgene is accompanied by spontaneous mutations in Nras, Kras, and Cbl. Blood 2008;112:2017-2019.

118. Sauvageau G, Thorsteinsdottir U, Hough MR et al. Overexpression of HOXB3 in hematopoietic cells causes defective lymphoid development and progressive myeloproliferation. Immunity 1997;6:13-22.

119. Greer JM, Puetz J, Thomas KR, Capecchi MR. Maintenance of functional equivalence during paralogous Hox gene evolution. Nature 2000;403:661665. 
120. lacovino $M$, Hernandez $C, X u Z$ et al. A conserved role for Hox paralog group 4 in regulation of hematopoietic progenitors. Stem Cells Dev. 2009;18:783-792.

121. Daga A, Podesta M, Capra MC et al. The retroviral transduction of HOXC4 into human CD34(+) cells induces an in vitro expansion of clonogenic and early progenitors. Exp. Hematol. 2000;28:569-574.

122. Bjornsson JM, Larsson N, Brun AC et al. Reduced proliferative capacity of hematopoietic stem cells deficient in Hoxb3 and Hoxb4. Mol.Cell Biol. 2003;23:3872-3883.

123. Kissinger CR, Liu BS, Martin-Blanco E, Kornberg TB, Pabo CO. Crystal structure of an engrailed homeodomain-DNA complex at 2.8 A resolution: a framework for understanding homeodomain-DNA interactions. Cell 1990;63:579-590.

124. Otting G, Qian YQ, Billeter $M$ et al. Protein--DNA contacts in the structure of a homeodomain--DNA complex determined by nuclear magnetic resonance spectroscopy in solution. EMBO J. 1990;9:3085-3092.

125. Chang CP, Shen WF, Rozenfeld S et al. Pbx proteins display hexapeptidedependent cooperative DNA binding with a subset of Hox proteins. Genes Dev. 1995;9:663-674.

126. Knoepfler PS, Kamps MP. The pentapeptide motif of Hox proteins is required for cooperative DNA binding with $\mathrm{Pbx} 1$, physically contacts $\mathrm{Pbx} 1$, and enhances DNA binding by Pbx1. Mol.Cell Biol. 1995;15:5811-5819.

127. Phelan ML, Rambaldi I, Featherstone MS. Cooperative interactions between HOX and PBX proteins mediated by a conserved peptide motif. Mol.Cell Biol. 1995;15:3989-3997.

128. Desplan C, Theis J, O'Farrell PH. The sequence specificity of homeodomain-DNA interaction. Cell 1988;54:1081-1090.

129. Pellerin I, Schnabel C, Catron KM, Abate C. Hox proteins have different affinities for a consensus DNA site that correlate with the positions of their genes on the hox cluster. Mol.Cell Biol. 1994;14:4532-4545.

130. Shen WF, Chang CP, Rozenfeld $S$ et al. Hox homeodomain proteins exhibit selective complex stabilities with Pbx and DNA. Nucleic Acids Res. 1996;24:898-906.

131. Zappavigna V, Sartori D, Mavilio F. Specificity of HOX protein function depends on DNA-protein and protein-protein interactions, both mediated by the homeo domain. Genes Dev. 1994;8:732-744. 
132. Vijapurkar U, Fischbach N, Shen $\mathrm{W}$ et al. Protein kinase C-mediated phosphorylation of the leukemia-associated HOXA9 protein impairs its DNA binding ability and induces myeloid differentiation. Mol.Cell Biol. 2004;24:3827-3837.

133. Bei L, Lu Y, Eklund EA. HOXA9 activates transcription of the gene encoding gp91Phox during myeloid differentiation. J.Biol.Chem. 2005;280:12359-12370.

134. Eklund EA, Goldenberg I, Lu Y, Andrejic J, Kakar R. SHP1 protein-tyrosine phosphatase regulates HoxA10 DNA binding and transcriptional repression activity in undifferentiated myeloid cells. J.Biol.Chem. 2002;277:3687836888.

135. Moens CB, Selleri L. Hox cofactors in vertebrate development. Dev.Biol. 2006;291:193-206.

136. Shen WF, Montgomery JC, Rozenfeld S et al. AbdB-like Hox proteins stabilize DNA binding by the Meis1 homeodomain proteins. Mol.Cell Biol. 1997;17:6448-6458.

137. Shanmugam K, Green NC, Rambaldi I, Saragovi HU, Featherstone MS. PBX and MEIS as non-DNA-binding partners in trimeric complexes with HOX proteins. Mol.Cell Biol. 1999;19:7577-7588.

138. Neuteboom ST, Murre C. Pbx raises the DNA binding specificity but not the selectivity of antennapedia Hox proteins. Mol.Cell Biol. 1997;17:4696-4706.

139. Eklund EA, Jalava A, Kakar R. Tyrosine phosphorylation of HoxA10 decreases DNA binding and transcriptional repression during interferon gamma-induced differentiation of myeloid leukemia cell lines. J.Biol.Chem. 2000;275:20117-20126.

140. Beslu N, Krosl J, Laurin M et al. Molecular interactions involved in HOXB4induced activation of HSC self-renewal. Blood 2004;104:2307-2314.

141. Svingen T, Tonissen KF. Hox transcription factors and their elusive mammalian gene targets. Heredity 2006;97:88-96.

142. Theokli C, Morsi El-Kadi AS, Morgan R. TALE class homeodomain gene Irx5 is an immediate downstream target for Hoxb4 transcriptional regulation. Dev.Dyn. 2003;227:48-55.

143. Moris E, Reiden P, Durston A, Morgan R. The small GTPase Rap1 is an immediate downstream target for Hoxb4 transcriptional regulation. Mech.Dev. 2002;113:131-139. 
144. Schiedlmeier B, Santos AC, Ribeiro A et al. HOXB4's road map to stem cell expansion. Proc.Natl.Acad.Sci.U.S.A 2007;104:16952-16957.

145. Nakano T. Hematopoietic stem cells: generation and manipulation. Trends Immunol. 2003;24:589-594.

146. Kyba M, Perlingeiro RC, Daley GQ. HoxB4 confers definitive lymphoidmyeloid engraftment potential on embryonic stem cell and yolk sac hematopoietic progenitors. Cell 2002;109:29-37.

147. Thorsteinsdottir U, Sauvageau G, Humphries RK. Enhanced in vivo regenerative potential of HOXB4-transduced hematopoietic stem cells with regulation of their pool size. Blood 1999;94:2605-2612.

148. Zhang XB, Beard BC, Beebe $\mathrm{K}$ et al. Differential effects of HOXB4 on nonhuman primate short- and long-term repopulating cells. PLoS.Med. 2006;3:e173.

149. Buske C, Feuring-Buske M, Abramovich $C$ et al. Deregulated expression of HOXB4 enhances the primitive growth activity of human hematopoietic cells. Blood 2002;100:862-868.

150. Schmittwolf C, Porsch M, Greiner A, Avots A, Muller AM. HOXB4 confers a constant rate of in vitro proliferation to transduced bone marrow cells. Oncogene 2005;24:561-572.

151. Helgason CD, Sauvageau G, Lawrence HJ, Largman C, Humphries RK. Overexpression of HOXB4 enhances the hematopoietic potential of embryonic stem cells differentiated in vitro. Blood 1996;87:2740-2749.

152. Chan KM, Bonde S, Klump H, Zavazava N. Hematopoiesis and immunity of HOXB4-transduced embryonic stem cell-derived hematopoietic progenitor cells. Blood 2008;111:2953-2961.

153. Klump H, Schiedlmeier B, Baum C. Control of self-renewal and differentiation of hematopoietic stem cells: HOXB4 on the threshold. Ann.N.Y.Acad.Sci. 2005;1044:6-15.

154. Schiedlmeier B, Klump H, Will E et al. High-level ectopic HOXB4 expression confers a profound in vivo competitive growth advantage on human cord blood CD34+ cells, but impairs lymphomyeloid differentiation. Blood 2003;101:1759-1768.

155. Zhang XB, Schwartz JL, Humphries RK, Kiem HP. Effects of HOXB4 overexpression on in vitro expansion and immortalization of hematopoietic cells from different species. Stem Cells 2007;25:2074-2081. 
156. Pilat S, Carotta S, Schiedlmeier B et al. HOXB4 enforces equivalent fates of ES-cell-derived and adult hematopoietic cells. Proc.Natl.Acad.Sci.U.S.A 2005; 102:12101-12106.

157. Zhang $X B$, Beard $B C$, Trobridge GD et al. High incidence of leukemia in large animals after stem cell gene therapy with a HOXB4-expressing retroviral vector. J.Clin.Invest. 2008;118:1502-1510.

158. Larochelle A, Choi U, Shou $Y$ et al. In vivo selection of hematopoietic progenitor cells and temozolomide dose intensification in rhesus macaques through lentiviral transduction with a drug resistance gene. J.Clin.Invest. 2009;119:1952-1963.

159. Brun AC, Bjornsson JM, Magnusson M et al. Hoxb4-deficient mice undergo normal hematopoietic development but exhibit a mild proliferation defect in hematopoietic stem cells. Blood 2004;103:4126-4133.

160. Bijl J, Thompson A, Ramirez-Solis R et al. Analysis of HSC activity and compensatory Hox gene expression profile in Hoxb cluster mutant fetal liver cells. Blood 2006;108:116-122.

161. Magnusson M, Brun AC, Lawrence HJ, Karlsson S. Hoxa9/hoxb3/hoxb4 compound null mice display severe hematopoietic defects. Exp.Hematol. 2007;35:1421-1428.

162. Kostic D, Capecchi MR. Targeted disruptions of the murine Hoxa-4 and Hoxa-6 genes result in homeotic transformations of components of the vertebral column. Mech.Dev. 1994;46:231-247.

163. Saegusa H, Takahashi N, Noguchi S, Suemori H. Targeted disruption in the mouse Hoxc-4 locus results in axial skeleton homeosis and malformation of the xiphoid process. Dev.Biol. 1996;174:55-64.

164. Horan GS, Kovacs EN, Behringer RR, Featherstone MS. Mutations in paralogous Hox genes result in overlapping homeotic transformations of the axial skeleton: evidence for unique and redundant function. Dev.Biol. 1995;169:359-372.

165. Sanyal M, Tung JW, Karsunky $\mathrm{H}$ et al. B-cell development fails in the absence of the Pbx1 proto-oncogene. Blood 2007;109:4191-4199.

166. Selleri L, Depew MJ, Jacobs $Y$ et al. Requirement for Pbx1 in skeletal patterning and programming chondrocyte proliferation and differentiation. Development 2001;128:3543-3557.

167. lacovino M, Hernandez $\mathrm{C}, \mathrm{Xu} Z \mathrm{Z}$ et al. A conserved role for Hox paralog group 4 in regulation of hematopoietic progenitors. Stem Cells Dev. 2008;18:783-792. 
168. Krosl J, Beslu N, Mayotte N, Humphries RK, Sauvageau G. The competitive nature of HOXB4-transduced HSC is limited by PBX1: the generation of ultra-competitive stem cells retaining full differentiation potential. Immunity 2003;18:561-571.

169. Cellot $\mathrm{S}, \mathrm{Kros} \mathrm{J}$, Chagraoui $\mathrm{J}$ et al. Sustained in vitro trigger of self-renewal divisions in Hoxb4hiPbx1(10) hematopoietic stem cells. Exp. Hematol. 2007;35:802-816.

170. Yang LV, Nicholson RH, Kaplan J, Galy A, Li L. Hemogen is a novel nuclear factor specifically expressed in mouse hematopoietic development and its human homologue EDAG maps to chromosome 9q22, a region containing breakpoints of hematological neoplasms. Mech.Dev. 2001;104:105-111.

171. Yang LV, Heng HH, Wan J et al. Alternative promoters and polyadenylation regulate tissue-specific expression of Hemogen isoforms during hematopoiesis and spermatogenesis. Dev.Dyn. 2003;228:606-616.

172. Li CY, Zhan YQ, Xu CW et al. EDAG regulates the proliferation and differentiation of hematopoietic cells and resists cell apoptosis through the activatin of nuclear factor-kappa B. Cell Death.Differ. 2004;11:1299-1308.

173. Li CY, Zhan YQ, Li W et al. Overexpression of a hematopoietic transcriptional regulator EDAG induces myelopoiesis and suppresses lymphopooiesis in transgenic mice. Leukemia 2007;21:2277-2286.

174. Zhou $Y, X u$ WX, Zhan YQ et al. Expression of EDAG-1 gene in human leukemia and lymphoma cell lines. Ai Zheng 2004;23:1238-1243.

175. An LL, Li G, Wu KF et al. High expression of EDAG and its significance in AML. Leukemia 2005;19:1499-1502.

176. Lu J, Xu WX, Wang SY et al. Overexpression of EDAG-1 in NIH3T3 cells leads to malignant transformation. Sheng Wu Hua Xue Yu Sheng Wu Wu Li Xue Bao 2002;34:95-98.

177. Sawai N, Zhou S, Vanin EF et al. Protection and in vivo selection of hematopoietic stem cells using temozolomide, O6-benzylguanine, and an alkyltransferase-expressing retroviral vector. Mol.Ther. 2001;3:78-87.

178. Marx JC, Allay JA, Persons DA et al. High-efficiency transduction and longterm gene expression with a murine stem cell retroviral vector encoding the green fluorescent protein in human marrow stromal cells. Hum.Gene Ther. 1999;10:1163-1173. 
179. Markowitz D, Goff S, Bank A. A safe packaging line for gene transfer: separating viral genes on two different plasmids. J.Virol. 1988;62:11201124.

180. Persons DA, Mehaffey MG, Kaleko M, Nienhuis AW, Vanin EF. An improved method for generating retroviral producer clones for vectors lacking a selectable marker gene. Blood Cells Mol.Dis. 1998;24:167-182.

181. Shou $Y, M a Z$, Lu T, Sorrentino BP. Unique risk factors for insertional mutagenesis in a mouse model of XSCID gene therapy. Proc.Natl.Acad.Sci.U.S.A 2006;103:11730-11735.

182. Stepanova L, Sorrentino BP. A limited role for p16Ink4a and p19Arf in the loss of hematopoietic stem cells during proliferative stress. Blood 2005;106:827-832.

183. Indra AK, Warot X, Brocard J et al. Temporally-controlled site-specific mutagenesis in the basal layer of the epidermis: comparison of the recombinase activity of the tamoxifen-inducible Cre-ER(T) and Cre-ER(T2) recombinases. Nucleic Acids Res. 1999;27:4324-4327.

184. Yang LV, Wan J, Ge Y et al. The GATA site-dependent hemogen promoter is transcriptionally regulated by GATA1 in hematopoietic and leukemia cells. Leukemia 2006;20:417-425.

185. Bodine DM, McDonagh KT, Seidel NE, Nienhuis AW. Survival and retrovirus infection of murine hematopoietic stem cells in vitro: effects of 5FU and method of infection. Exp.Hematol. 1991;19:206-212.

186. Harrison DE, Lerner CP. Most primitive hematopoietic stem cells are stimulated to cycle rapidly after treatment with 5-fluorouracil. Blood 1991;78:1237-1240.

187. Domen $\mathrm{J}$. The role of apoptosis in regulating hematopoiesis and hematopoietic stem cells. Immunol.Res. 2000;22:83-94.

188. Opferman JT, Iwasaki H, Ong CC et al. Obligate role of anti-apoptotic MCL1 in the survival of hematopoietic stem cells. Science 2005;307:1101-1104.

189. Milsom MD, Duxbury R, Gagen D et al. Overexpression of HOXB4 confers a myelo-erythroid differentiation delay in vitro. Leukemia 2005;19:148-153.

190. Wiederschain D, Chen L, Johnson B et al. Contribution of polycomb homologues Bmi-1 and Mel-18 to medulloblastoma pathogenesis. Mol.Cell Biol. 2007;13:4968-4979. 
191. Fasano CA, Dimo JT, Ivanova NB et al. shRNA knockdown of Bmi-1 reveals a critical role for $\mathrm{p} 21-\mathrm{Rb}$ pathway in NSC self-renewal during development. Cell Stem Cell 2007;1:87-99.

192. Takeshita M, Ichikawa M, Nitta E et al. AML1-Evi-1 specifically transforms hematopoietic stem cells through fusion of the entire Evi-1 sequence to AML1. Leukemia 2008;22:1241-1249.

193. Liu H, Holm M, Xie X et al. AML1/Runx1 recruits calcineurin to regulate granulocyte macrophage colony-stimulating factor by Ets1 activation. J.Biol. Chem. 2004;279:29398-29408.

194. Goyama S, Yamamoto G, Shimabe M et al. Evi-1 is a critical regulator for hematopoietic stem cells and transformed leukemia cells. Cell Stem Cell 2008;3:207-220.

195. Krosl J, Austin P, Beslu N et al. In vitro expansion of hematopoietic stem cells by recombinant TAT-HOXB4 protein. Nature Medicine 2003;9:14281432.

196. Park PJ. ChIP-seq: advantages and challenges of a maturing technology. Nature Reviews Genetics 2009;10:669-680.

197. Oguro H, Iwama A. Life and death in hematopoietic stem cells. Curr.Opin.Immunol. 2007;19:503-509.

198. Opferman JT. Life and death during hematopoietic differentiation. Curr.Opin.Immunol. 2007;19:497-502.

199. Veis DJ, Sorenson CM, Shutter JR, Korsmeyer SJ. Bcl-2-deficient mice demonstrate fulminant lymphoid apoptosis, polycystic kidneys, and hypopigmented hair. Cell 1993;75:229-240.

200. Motoyama N, Wang F, Roth KA et al. Massive cell death of immature hematopoietic cells and neurons in Bcl-x-deficient mice. Science 1995;267:1506-1510.

201. Vaux DL, Cory S, Adams JM. Bcl-2 gene promotes haemopoietic cell survival and cooperates with c-myc to immortalize pre-B cells. Nature 1988;335:440-442.

202. Rusten LS, Smeland EB, Jacobsen FW et al. Tumor necrosis factor-alpha inhibits stem cell factor-induced proliferation of human bone marrow progenitor cells in vitro. Role of p55 and p75 tumor necrosis factor receptors. J.Clin.Invest. 1994;94:165-172. 
203. Cheng $\mathrm{T}$, Rodrigues $\mathrm{N}$, Shen $\mathrm{H}$ et al. Hematopoietic stem cell quiescence maintained by $\mathrm{p} 21$ cip1/waf1. Science 2000;22:5506-5517.

204. Cheng T, Rodrigues N, Dombkowski D, Stier S, Scadden DT. Stem cell repopulation efficiency but not pool size is governed by p27(kip1). Nat. Med. 2000;6:1235-1240.

205. Yuan Y, Shen H, Franklin DS, Scadden DT, Cheng T. In vivo self-renewing divisions of haematopoietic stem cells are increased in the absence of the early G1-phase inhibitor, p18INK4C. Nat.Cell Biol. 2004;6:436-442.

206. Giannola DM, Shlomchik WD, Jegathesan M et al. Hematopoietic expression of Hoxb4 is regulated in normal and leukemic stem cells through transcriptional activation of the Hoxb4 promoter by upstream stimulating factor (Usf)-1 and Usf-2. J.Clin.Invest. 2000;192:1479-1490.

207. Kirito K, Fox N, Kaushansky K. Thrombopoietin stimulates Hoxb4 expression: an explanation for the favorable effects of TPO on hematopoietic stem cells. Blood 2003;102:3172-3178.

208. Zhu J, Giannola DM, Zhang Y et al. NF-Y cooperates with USF1/2 to induce the hematopoietic expression of HOXB4. Blood 2003;102:24202427.

209. Dzierzak E, Medvinsky A. Mouse embryonic hematopoiesis. Trends Genet. 1995;11:359-366.

210. Robin C, Ottersbach K, Durand C et al. An unexpected role for IL-3 in the embryonic development of hematopoietic stem cells. Developmental Cell 2006;11:171-180.

211. Gehring WJ, Qian $Y Q$, Billeter $M$ et al. Homeodomain-DNA recognition, Cell 1994;78:211-223.

212. Mann RS, Lelli KM, Joshi R. Chapter 3 Hox specificity: unique roles for cofactors and collaborators. Current Topics in Developmental Biology 2009;88:63-106.

213. Vincent JP, Kassis JA, O'Farrell PH. A synthetic homeodomain binding site acts as a cell type specific, promoter specific enhancer in Drosophila embryos. EMBO J. 1990;9:2573-2578.

214. Zhang $X B$, Neff $T$, Thomasson $B$ et al. Ex vivo and in vivo expansion of HoxB4-Transduced CD $34^{+}$cells from dogs, baboons, macaques and humans. Molecular Therapy 2004;9:268-275. 
215. Pawliuk R, Eaves C, Humphries RK. Evidence of both ontogeny and transplant dose-regulated expansion of hematopoietic stem cells in vivo. Blood 1996;88:2852-2858.

216. Iscove NN, Nawa K. Hematopoietic stem cells expand during serial transplantation in vivo without apparent exhaustion. Curr.Biol. 1997;7:805808.

217. Calvi LM et al. Osteoblastic cells regulate the haematopoietic stem cell niche. Nature 2003;425:841-846.

218. Zhang $\mathrm{J}$ et al. Identification of the haematopoietic stem cell niche and control of the niche size. Nature 2003;425:836-841.

219. Thorsteinsdottir U, Sauvageau G, Hough MR et al. Overexpression of HOXA10 in murine hematopoietic cells perturbs both myeloid and lymphoid differentiation and leads to acute myeloid leukemia. Mol.Cell Biol. 1997; 17:495-505.

220. Lin YW, Aplan PD. Leukemic transformation. Cancer Biol.Ther. 2004;3:1320.

221. Aldrich MC, Zhang L, Wiemels $\mathrm{JL}$ et al. Cytogenetics of hispanic and white children with acute lymphoblastic leukemia in California. Cancer Epidemiol. Biomarkers Prev. 2006;15:578-581.

222. Pui $\mathrm{CH}$, Relling MV, Downing JR. Acute lymphoblastic leukemia. N.Engl.J. Med. 2004;350:1535-1548.

223. Thakar MS, Zhang XB, Beard BC et al. Transmission and expansion of HOXB4-induced leukemia in two immunosuppressed dogs: implication for a new canine leukemia model. Exp.Hematol. 2009;10:1157-1166.

224. Larochelle A, Dunbar CE. HOXB4 and retroviral vectors: adding fuel to the fire. J.Clin.Invest. 2008;118:1350-1353. 


\section{VITA}

Jie Jiang was born in Huangshan, Anhui Province at P.R.China in 1981. In 1998, she enrolled in the Department of Biochemistry in the School of Life Science at Nanjing University. After achieving her Bachelor's degree in 2002, she continued her study in biochemistry and molecular sciences at Nanjing University and got her Master's degree in 2004. In September of the same year, she chose to enter the Integrated Program in Biomedical Sciences at the University of Tennessee Health Science Center for PhD studies. In May 2005, she joined Dr. Brian P. Sorrentino's laboratory at St. Jude Children's Research Hospital and conducted research in the mechanisms of HOXB4 function in promoting hematopoietic stem and progenitor cell self-renewal. She is expected to finish her work in December 2009. 